\title{
Checklist of floral species at the Institute of Forestry, Pokhara Campus, Pokhara, Nepal
}

\author{
Mahamad Sayab Miya ${ }^{1}$ and Deepak Gautam ${ }^{1^{*}}$ \\ 1 Institute of Forestry, Tribhuvan University, Pokhara Campus, Pokhara, Nepal
}

\section{KEYWORDS}

\section{Asteraceae \\ CITES}

IAPS

Fabaceae

Flora

\begin{abstract}
Plants are a component of biodiversity and support life system on earth. Exploration of plant species is important to aquire knowledge on plant biodiversity and their distribution. This study documents floral species in the Institute of Forestry, Pokhara Campus, Nepal. The Campus is one of the academic institutions for producing forestry graduates. The plant species were listed from 2020 to the first half of the year 2021 by walking along the accessible routes inside the Campus. A total of 331 species of plants from 276 genera and 99 families were recorded. Fabaceae family represented the maximum number of plant species $(n=26)$ followed by Asteraceae $(n=25)$ and Poaceae $(n=21) .43 \%$ of the total species recorded were herbs. $31 \%$ of the total species were trees. 16 species were invasive alien plant species (IAPS) and four species are listed under Appendix-II of CITES.
\end{abstract}

\section{Introduction}

Plants (Flora) are integral parts of biodiversity and ecosystems. Plants on the land originated about 480-360 million years ago in the midPalaeozoic era (Kenrick and Crane 1997) from freshwater algae (Sakakibara 2016). Out of the 5-30 million estimated plant species worldwide, only $1.2-1.9$ million species (5-10\%) have been identified, named, and classified so far (Govaerts 2001; Prance 2001; Guerra Garcia et al. 2008; Kunzig 2008; Mora et al. 2011). It is estimated that there could be 4 million flowering plants on the earth (Govaerts 2001; Shrestha 2011). The maximum floral diversity is present in tropical and subtropical regions (WCMC 1992).

Nepal is a small mountainous country occupying only $0.1 \%$ of the global land. Due to the variable geography and climate, it is rich in biodiversity. It harbors 118 types of ecosystems, $3.2 \%$ of the world's known flora (GoN/MoFSC 2014). Nepal ranks 31st in the world and 10th in Asia in terms of flowering plant diversity. Floristic exploration was initiated in the early 
nineteenth century in Nepal from eastern parts (Rajbhandari 1976). Since then, many researchers have been documenting the flora of Nepal (Rajbhandari 2016). GoN/MoFSC (2014) has presented 6,973 species, Kunwar et al. (2010) has reported 6,653 species while Rajbhandari et al. (2017) reported 5,309 species of flowering plants existing in Nepal. However, the species number may increase up to 7,000 after the revision of the total list (Shrestha and Bajracharya 2019). It is estimated that there are about 1,001 species of algae (Prasad 2013), fungi- 2,182 species (Kost and Adhikari 2015), lichens -850 species (Søchting 2015), bryophytes- 1,213 species (Pradhan 2016), pteridophytes- 550 species (Fraser-Jenkins et al. 2015), and gymnosperms -41 species (Shrestha et al. 2018) in Nepal. About 312 species of flowering plants are endemic to Nepal (Rajbhandari et al. 2017); followed by fungi- 131 species (Adhikari 2016); lichens48 species (Bhuju et al. 2007), bryophytes-30 species (Pradhan 2016), and algae- 12 species (Baral 1995).

Human beings depend on plants resource to fulfill their basic needs of food, clothes, shelter and health care (Bhattarai and Khadka 2016; Bhattarai 2019; Miya et al. 2020; Rajbhandary et al.2020). Plants are also used for herbal medicine, construction materials, fuelwood, fodder, fiber and social religious purposes (Rajbhandary and Winkler 2015). A large proportion of the rural population depend on plant resources (such as timber, non-timber forest products (NTFPs) and medicinal and aromatic plants (MAPs) to sustain their livelihoods and economy (Giri et al. 2001; Angelsen et al. 2014; Shackleton and Pullanikkatil 2019; Lamichhane et al. 2021). Plants play important roles in ecosystem balance, oxygen balance, carbon sequestration, control erosion and nutrient recycling (Rajbhandary et al. 2020).

There is a growing concern about floristic composition, species richness and forest structure worldwide as forests are being fragmented (Myers et al. 2000). The major threats to biodiversity are habitat degradation, fragmentation and conversion of forests (Abramovitz 1998) which ultimately cause the loss of floral species and ecosystem imbalance. Plant diversity is also threatened by deforestation, climate change, natural disasters, invasion of alien species, overgrazing and human interventions (Hill and Curran 2003; Laidlaw et al. 2007; Murphy et al. 2013; Ribeiro et al. 2014; Thapa and Maharjan 2014; Chaudhary et al. 2016). Many plant species are threatened in Nepal for example, Lauth Salla (Taxus wallichiana) and Sarpagandha (Rauvolfia serpentina) (Joshi et al. 2017).

It is important to explore floral species in a particular area to identify vital elements of floral diversity, monitor and manage vegetation type, protect and conserve threatened plant species (Sahu and Dhal 2012; Georgieva et al. 2013; Akinyemi and Oke 2014). Bhatta (2011) has recorded a total of 112 species of plants from Institute of Forestry (IOF), Pokhara Campus (PC). The IOF-PC aims to produce capable, efficient, and practical based forest and natural resource technicians to address needs and problems in the field of forestry and related sectors (www.iofpc.edu.np ). The identification and knowledge of flora are essential for all forestry graduates. The campus itself has an isolated forest (urban forest) named Banpale Danda adjacent to the Campus buildings. This forest is rich in plant diversity and students are taught about plants found there through different plant identification programs. However, proper documentation of the floral species within the forest and campus premises is still lacking. This study thus aims to document floral species found in IOF-PC. The finding of the study would also help to insight and compare the floral diversity of isolated forests in different parts of the country.

\section{Materials and Methods}

\section{Study area}

This study was carried out in the Institute 
of Forestry (IOF), Pokhara Campus (PC), Pokhara, Nepal from 2020 to the first half of the year 2021. IOF-PC lies in the southern part of Pokhara Valley $\left(28.18^{\circ} \mathrm{N}, 83.99^{\circ} \mathrm{E}\right)$. It covers an area of 1528 ropani (77.77 ha) ( 1 ha $=19.66$ ropani). The altitude ranges from $750-915$ $\mathrm{m}$ (Figure 1). The forest (Banpale forest) is a mixed subtropical forest dominated by Schima wallichii (Chilaune) and Castonopsis indica (Katus). The forest is rich in both floral and faunal diversity. Recently, 10 species of bats were recorded in the forest (Bhattarai et al. 2021). IOF-PC is bounded by Seti Gorge to the west whereas the other sides are surrounded by agricultural land, grazing land, shrubland and human settlements. Pokhara valley receives $4,000 \mathrm{~mm}$ of precipitation in a year, however it varies by monsoon (June-September) $(<3,000$ $\mathrm{mm}$ ), winter (December-February) (about $100 \mathrm{~mm}$ ), pre-monsoon (March-May) $(<500$ $\mathrm{mm}$ ) and post monsoon (October-November) $(<150 \mathrm{~mm})$. Similarly, the mean annual air temperature is $21^{\circ} \mathrm{C}$. The mean temperature of winter, pre-monsoon, monsoon and postmonsoon are about $14^{\circ} \mathrm{C}, 22^{\circ} \mathrm{C}, 26^{\circ} \mathrm{C}$ and $23^{\circ} \mathrm{C}$, respectively. December, January and February are the driest months (Gautam et al. 2019).

\section{Data collection}

The floral species were listed along the accessible walking routes inside the forest and campus. The study area was also randomly explored to add species. All the floral species were photographed for identification. The plant species were identified in the fields by examining their leaf, flower and fruit structures. The identification knowledge was acquired from different plant identification programs. The unidentified species, as well as identified species were further confirmed with the help of literature (Polunin and Stainton 1984; Shrestha 1998; Press et al. 2000; Pearce and Cribb 2002; Obha et al. 2008; Rajbhandari and Adhikari
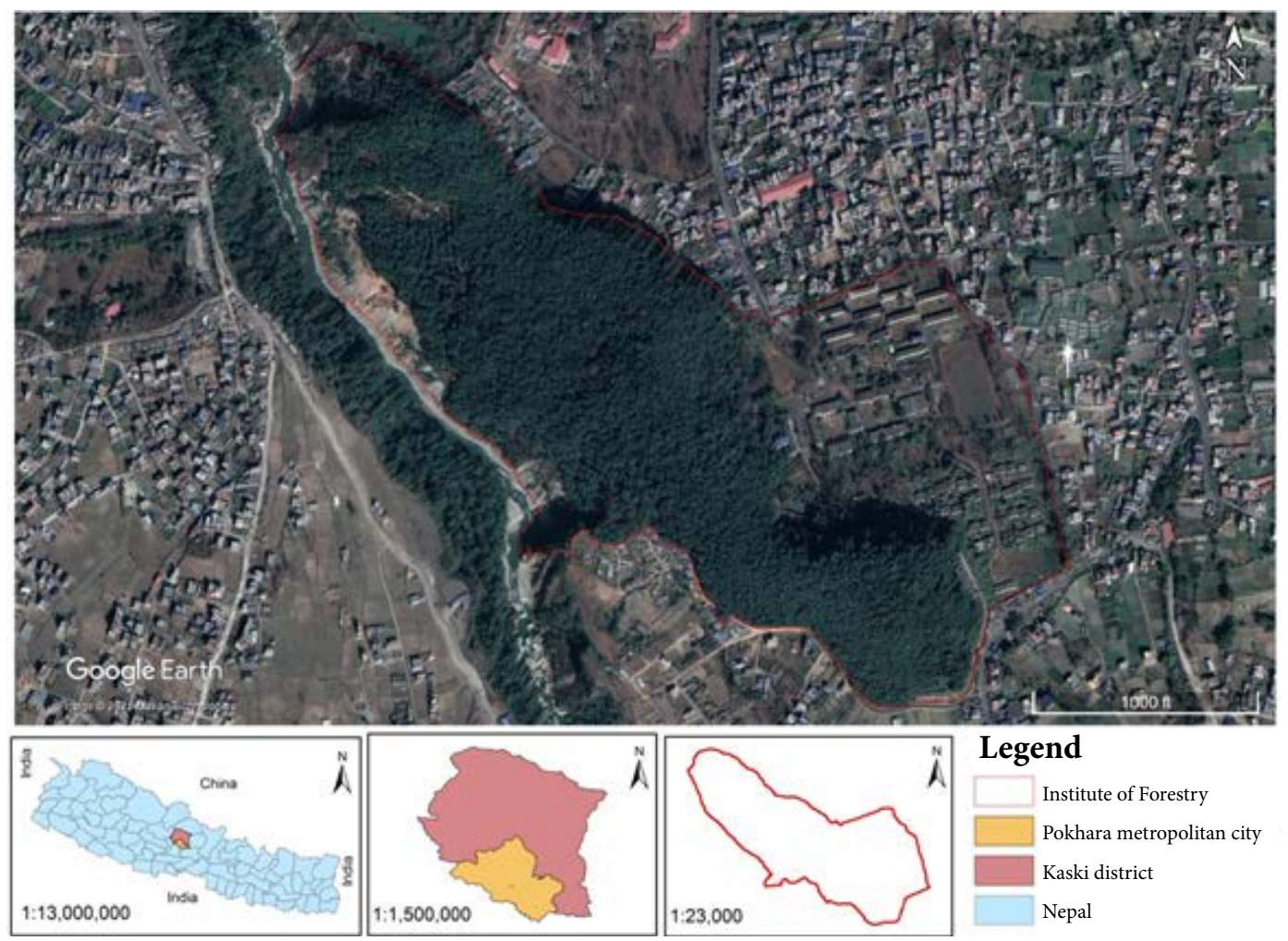

Figure 1: Map of study area showing Institute of Forestry, Pokhara Campus 
2009; Raskoti 2009; Rajbhandari and Dhungana 2010, 2011; Watson et al. 2011; Rokaya et al. 2013; Rajbhandary 2016; Shrestha et al. 2018), www.efloras.org, http://www.floraofnepal.org, and consultation with experts and campus staffs. The major uses of the species were listed through consultation with campus staff and presented into different use categories (Timber, fuelwood, fodder, ornamental, medicinal and others). The cultivation status is mentioned as wild and cultivated (Ornamental, planted and agriculture).

The nomenclature and author citation are based on the Catalogue of Life (2019). The photographs are presented in photo plates. The invasive species were categorized following Shrestha (2019). Likewise, CITES-listed species are categorized following Joshi et al. (2017). The native and introduced species status were based on KATH (2020) and POWO (2021).

\section{Data analysis}

The data were pooled in Excel and presented in tables. The plant species are listed alphabetically.

\section{Results and Discussion}

A total of 331 species of plants from 276 genera and 99 families were recorded in the study area. The plant species with their scientific name, local name, common name, family name, habit, cultivation status and native or introduced status and major uses are presented in Tables 1. The present study recorded about three times greater number of plant species than Bhatta (2011).

\section{Family-wise composition of the floral species}

The plant species were represented from 99 families. Family Fabaceae represented the maximum number of plant species $(n=26)$, followed by Asteraceae $(\mathrm{n}=25)$, Poaceae $(\mathrm{n}=$ $21)$, Moraceae $(\mathrm{n}=16)$, and Lamiaceae $(\mathrm{n}=$ 14) (Table 1). The maximum number of plant species from the family Fabaceae was also observed in different regions of Nepal (Dhami 2008; Pathak and Baniya 2017; Pandey and Ghimire 2020; Bhandari et al. 2021). Fabaceae is the 3rd largest plant family in the world, and possesses wide life forms such as trees, shrubs, herbs and climbers (Watson and Dallwitz 1992; Judd et al. 1999; Ahmed et al. 2016). Likewise, another family - Asteraceae which is the largest plant family comprising more than 24, 000 species (Funk et al. 2009; Valles et al. 2013), represented a quite similar species number to Fabaceae in the study area.

\section{Life forms and cultivation status of the floral species}

Off the total identified floral species, 43\% (n $=143$ ) of them were herbs, followed by trees $(31 \%, \mathrm{n}=101)$, shrubs $(20 \%, \mathrm{n}=66)$ and climbers $(6 \%, n=21)$. The herb species were mostly found in open areas and associated with shrubs, trees and climbers in the study area. Bhattarai (2019), Budha-Magar et al. (2020) and Bhandari et al. (2021) had also documented higher number of herb species in their studies in Nepal. A total of 176 species were recorded in the wild state and 155 species from cultivation state (Ornamental-75 species, Planted- 70 species and agriculture- 10 spcies) (Table 1).

\section{CITES listed floral species}

Out of the 154 CITES-listed plant species of Nepal (Joshi et al. 2017), four species (Dalbergia latifolia, Dalbergia sissoo, Euphorbia royleana and Rauvolfia serpentina) which are listed in Appendix-II of CITES were found in the study area. These species are facing higher risk of extinction due to overharvesting throughout the world (Dwianto et al. 2019; Kunwar 2019; Abdullah and Rafay 2021). Hence, to protect their illegal trade and save them from extinction, these species are listed under CITES-II. These species serve as examples of protected species inside the campus. 


\section{Native, introduced and invasive alien floral species}

Of the total, 193 species were native, 130 species were introduced and eight species were not specified their status (KATH 2020; POWO 2021). Out of the 26 naturalized invasive alien plant species found in Nepal (Shrestha 2019), 16 species were recorded from the study area. They are: Ageratina adenophora, Ageratum conyzoides, Ageratum houstonianum, Alternanthera philoxeroides, Amaranthus spinosus, Bidens pilosa, Chromolaena odorata, Eichhornia crassipes, Galinsoga quadriradiata, Lantana camara, Mimosa pudica, Oxalis latifolia, Parthenium hysterophorus, Senna occidentalis, Senna tora and Spermacoce alata. These invasive species are threats to plant diversity (Vitousek et al. 1997; Mooney and Hobbs 2000; Paini et al. 2016). These species were growing well in open areas, under the trees having low canopy cover and sides of the walking routes in the campus premises. The high number of butterflies could be seen on the flowers of species like; Lantana camera, Bidens pilosa, Ageratina adenophora, Ageratum conyzoides, and Ageratum houstonianum during the study period. These plant species might be beneficial for butterflies. However, these invasive species are gradually replacing native herbs and shrub species in the study area.

\section{Usefulness of the floral species}

Among the total species, about 68 species such as Achyranthes aspera, Azaderachta indica, Terminalia chebula, Bryophyllum pinnatum,
Cassia fistula etc. were used for medicinal proposes. 85 species of trees, shrubs and grasses (Ficus glaberrima, Ficus auriculata, Leucaena lecocephala etc.) were used for fodder, 15 species (Bombax ceiba, Dalbergia sissoo etc.) were used for timber, 81 species for ornamental, and other species were used for fuelwood, hedging, mulching, fruits, etc. Many floral species were found to be used for more than one proposes.

The presence of a large number of floral species in this small area might be due to the forest being conserved for study purposes, no grazing, no entry of local people and different plantation activities conducted around the campus premises which add new species. However, many mature tree species which are in few number are cut down which may cause loss of species diversity. Also access trails inside the forests may affect on ground vegetation diversity.

\section{Conclusion}

The study revealed that the Institute of Forestry, Pokhara Campus is rich (331 species) in floral diversity. The CITES-listed plants present there should be managed. The record of a large number of invasive alien plants species indicates the threats to native vegetation. Therefore, these invasive species should be managed with effective interventions to retain native plant diversity. This checklist will help to gain knowledge of floral species of this Campus, which we recommend to update at regular intervals. 


\section{References}

Abdullah, M., \& Rafay, M. (2021). Ecology of Dalbergia sissoo. In Dalbergia sissoo, pp. 1326.

Abramovitz, J. N., \& Gnan, P. (1998). Putting a value on nature's free' services. World Watch, 11(1), 10-19.

Adhikari, M. K. (2016). Revised checklist to the mycotaxa proposed from Nepal. Bulletin of Department of Plant Resource, 38, 1-11.

Ahmad, F., Anwar, F., \& Hira, S. (2016). Review on medicinal importance of Fabaceae family. Pharmacology online, 3, 151-157.

Akinyemi, D. S., \& Oke, S. O. (2014). Floristic composition and structural diversity of Shasha forest reserve in Ile-Ife, Southwestern Nigeria. Notulae Scientia Biologicae, 6(4), 433-440. doi: https://doi.org/10.15835/ nsb649373

Angelsen, A., Jagger, P., Babigumira, R., Belcher, B., Hogarth, N. J., Bauch, S., Borner, J., SmithHall, C., \& Wunder. S. (2014). Environmental income and rural livelihoods: a globalcomparative analysis. World Development, 64, S12-S28. doi: https://doi. org/10.1016/j. worlddev.2014.03.006

Baral, S. R. (1995). Enumeration of the Algae of Nepal. Biodiversity Profile Project, Publication No. 11. Department of National Parks \& Wildlife Conservation, Government of Nepal.

Bhandari, R., Miya, M. S., \& Timilsina, S. (2021). Status and Diversity of Terrestrial plants in Ghodaghodi Lake Complex, Nepal. International Journal of Applied Sciences and Biotechnology, 9(1), 38-44. doi: https://doi. org/10.3126/ijasbt.v9i1.35084

Bhatta, J. P. (2011). Species diversity and composition of Forest vegetation of Banpale danda forest, Kaski, Nepal. LAP LAMBERT Academic Publishing, 64p.

Bhattarai, K. R. (2019). Enumeration of the flowering plants of Singha Durbar Premises, Kathmandu, Nepal. Journal of Plant Resources, 17, 69-81.

Bhattarai, K. R., \& Khadka, M. K. (2016). Ethnobotanical survey of medicinal plants from Ilam District, East Nepal. Our Nature, 14(1), 78-91. doi: https://doi.org/10.3126/ on.v14i1.16444

Bhattarai, P. K., Sharma, B., Neupane, A., Kunwar, S., \& Dhungana, P. (2021). Bat diversity in the Banpale forest, Pokhara, Nepal during spring season. Journal of Threatened Taxa, 13(6),
18479-18489. doi: https://doi.org/10.11609/ jott.6263.13.6.18479-18489

Bhuju, U. R., Shakya, P. R., Basnet, T. B., \& Shrestha, S. (2007). Nepal Biodiversity Resource Book: Protected Areas, Ramsar Sites and World Heritage Sites. International Centre for Integrated Mountain Development, Kathmandu, Nepal, 128p.

Budha-Magar, S., Bhandari, P., \& Ghimire, S. K. (2020). A Checklist of Flowering Plants of Jaljalâ Mountain and adjoining Areas, Rolpâ District, Western Nepal. Journal of Plant Resources, 18(1), 82-101.

Catalogue of life. (2019). Indexing the known species of World. Published in www.catalogueoflife. org (accessed on 27 May 2021)

Chaudhary, R. P., Uprety, Y., \& Rimal, S. K. (2016). Deforestation in Nepal: Causes, consequences and responses. Biological and Environmental hazards, risks, and disasters, 335-372. doi: http://dx.doi.org/10.1016/ B978-0-12-394847-2.00020-6

Dhami, N. (2008). Ethnomedicinal uses of plants is Western Terai of Nepal: A case study of Dekhatbhuli VDC of Kanchanpur district. Medicinal plants in Nepal: an anthology of contemporary research, 165-177.

Dwianto, W., Bahanawan, A., Kusumah, S. S., Darmawan, T., Amin, Y., Pramasari, D. A., Lestari, E., Akbar, F., \& Sudarmanto. (2019). Study on the existence and characteristics of Sonokeling (Dalbergia latifolia Roxb) as an Appendix II CITES Wood. In IOP Conference Series: Earth and Environmental Science, 374(1), 012063p.

Fraser-Jenkins, C. R., Kandel, D. R., \& Pariyar, S. (2015). Ferns and Fern-allies of Nepal Volume 1. National Herbarium and Plant Laboratories, Department of Plant Resources. Ministry of Forests and Soil Conservation, Kathmandu, Nepal. 492p.

Funk, V.A, Susanna, A., Stuessy, T. F., Bayer, R. J., \& editors. (2009). Systematics, evolution and biogeography of Compositae. Vienna International Association for Plant Taxonomy.

Gautam, D., Bhattarai, S., Sigdel, R., Jandug, C. M. B., Mujahid, A., \& GC, D. B. (2019). Climatic variability and wetland resources in Rupa Lake Catchment, Nepal. North American Academic Research, 2(12), 82-91. doi: https:// doi.org/10.5281/zenodo.3568477

Georgieva, N., Pachedjieva, K., \& Lyubenova, M. (2013). SCI "Zapadna Stara Planina and 
Predbalkan"-floristic studies on xerothermic oak forests. Bulgarian Journal of Agricultural Science, 19(2), 218-221.

Giri, T. K., Bhattacharya, P., \& Santra, S. C. (2001). Ecology and economy of NTFPs in village life-A case study from Bankura District in West Bengal, India. Annales of Forestry, 9(1), 1-16.

GoN/MoFSC. (2014). Nepal National Biodiversity Strategy and Action Plan 2014-2020. Ministry of Forest and Soil Conservation (MFSC), Kathmandu, Nepal.

Govaerts, R. (2001). How many species of seed plants are there?. Taxon, 50(4), 1085-1090. doi: https://doi.org/10.2307/1224723

Guerra García, J. M., Espinosa Torre, F., \& García Gómez, J. C. (2008). Trends in taxonomy today: an overview about the main topics in taxonomy. Zoológica baetica, 19, 15-49. doi: http://hdl.handle.net/11441/28285

Hill, J. L., \& Curran, P. J. (2003). Area, shape and isolation of tropical forest fragments: effects on tree species diversity and implications for conservation. Journal of biogeography, 30(9), 1391-1403. doi: https://doi.org/10.1046/ j.1365-2699.2003.00930.x

Joshi, N., Dhakal, K. S., \& Saud, D. S. (2017). Checklist of CITES Listed Flora of Nepal. Department of Plant Resources (DPR), Thapathali, Kathmandu, Nepal.

Judd, W. S., Campbell, C. S., Kellogg, E. A., Stevens, P. F. \& Donoghue, M. J. (1999). Plant systematics: a phylogenetic approach. Ecología mediterránea, 25(2), 215. https:// digitalcommons.library.umaine.edu/fac monographs/76

KATH (National Herbarium and Plant Laboratories). (2020). Alien Flora of Nepal at KATH. Version 1.3. Department of Plant Resources, MoFE, Government of Nepal. Occurrence dataset. https://doi.org/10.15468/enyzfx (accessed on 29 September 2021)

Kenrick, P., \& Crane, P. R. (1997). The origin and early evolution of plants on land. Nature, 389(6646), 33-39. doi: https://doi. org $/ 10.1038 / 37918$

Kost, G., \& Adhikari, M. K. (2015). Mycota. In: Nepal: An Introduction to the Natural History, Ecology and Human Environment of the Himalayas, Miehe, G., Pendry, C. A. \& Chaudhary, R. (eds.), Edinburgh: Royal Botanic Garden Edinburgh. pp. 203-210.

Kunwar, B. B. (2019). Establishing in situ gene bank of Rauvolfia serpentina (L.) Benth ex Kurtz in
Western Nepal with a focus on conservation and sustainability. Biodiversity International Journal, 3(4), 139-143.

Kunwar, R., Shrestha, M. K., Dhungana, S. K., Shrestha, P. R, Shrestha, K. K. (2010). Floral Biodiversity of Nepal: an update. Journal of Natural History Museum, 25, 295-311.

Kunzig, R. (2008). Are Hotspots the Key to Conservation. Scientific American, 18(4), 42-49. doi: https://doi.org/10.1038/ scientificamericanearth0908-42

Laidlaw, M., Kitching, R., Goodall, K., Small, A., \& Stork, N. (2007). Temporal and spatial variation in an Australian tropical rainforest. Austral Ecology, 32(1), 10-20. doi: https://doi. org/10.1111/j.1442-9993.2007.01739.x

Lamichhane, R., Gautam, D., Miya, M. S., Raut Chhetri, H. B., \& Timilsina, S. (2021). Role of Non-Timber Forest Products in Local Economy: A Case of Jajarkot District, Nepal. Grassroots Journal of Natural Resources, 4(1), 94-105.doi: https://doi.org/10.33002/ nr2581.6853.040107

Miya, M. S., Timilsina, S., \& Chhetri, A. (2020). Ethnomedicinal uses of plants by major ethnic groups of Hilly Districts in Nepal: A review. Journal of Medicinal Botany, 4, 2437. doi: https://doi.org/10.25081/jmb.2020. v4.6389

Mooney, H.A., \& Hobbs, R. J. (eds.). (2000). Invasive Species in a Changing World. Island Press, Washington, D.C.

Mora, C., Tittensor, D. P., Adl, S., Simpson, A. G., \& Worm, B. (2011). How many species are there on Earth and in the ocean? PLoS biology, 9(8), e1001127. doi: https://doi.org/10.1371/ journal.pbio. 1001127

Murphy, S. T., Subedi, N., Jnawali, S. R., Lamichhane, B. R., Upadhyay, G. P., Kock, R., \& Amin, R. (2013). Invasive mikania in Chitwan National Park, Nepal: the threat to the greater one-horned rhinoceros Rhinoceros unicornis and factors driving the invasion. Oryx, 47(3), 361-368. doi: https://doi. org/10.1017/S003060531200124X

Myers, N., Mittermeier, R. A., Mittermeier, C. G., Da Fonseca, G. A., \& Kent, J. (2000). Biodiversity hotspots for conservation priorities. Nature, 403(6772), 853-858. doi: https://doi. org/10.1038/35002501

Ōba, H., Iokawa, Y., \& Śarmā, L. (2008). Flora of Mustang, Nepal. Kodansha Scientific.

Paini, D. R., Sheppard, A. W., Cook, D. C., De Barro, P. J., Worner, S. P., \& Thomas, M. B. (2016). 
Global threat to agriculture from invasive species. Proceedings of the National Academy of Sciences, 113(27), 7575-7579. doi: https:// doi.org/10.1073/pnas.1602205113

Pandey, N., \& Ghimire, S. K. (2020). Floristic Diversity in a Community Managed Forest of Kanchanpur District, Western Nepal. Journal of Plant Resource, 18(1), 124-134.

Pathak, R. P., \& Baniya, C. B. (2017). Species diversity and tree carbon stock pattern in a community-managed tropical shorea forest in Nawalparasi, Nepal. International Journal of Ecology and Environmental Sciences, 42(5), 3-17.

Pearce, N. R. \& Cribb, P. J. (2002). Flora of Bhutan. Vol. 3, Part 3. Edinburgh, UK: Royal Botanic Garden.

Polunin, O., \& Stainton, J. D. A. (1984). Flowers of the Himalaya. New Delhi, India: Oxford University Press.

POWO. (2021). Plants of the World Online. Facilitated by the Royal Botanic Gardens, Kew. Published on the Internet. http://www. plantsoftheworldonline.org (accessed on 29 September 2021)

Pradhan, N. (2016). Bryophytes of Nepal. In: Frontiers of Botany, Jha, P.K., Siwakoti, M. and Rajbhandary, S. (eds.). Central Department of Botany, Tribhuvan University, Kathmandu, pp. 100-123.

Prance, G. T. (2001). Systematics: Relevance to the Twenty-first Century. e LS. doi: https://doi. org/10.1038/npg.els.0003608

Prasad, V. (2013). Biodiversity: Algae. In: Biological Diversity and Conservation, Jha, P. K., Neupane, F. P., Shrestha, M. L., \& Khanal, I. P. (eds.). Nepalpedia Series No.2. NAST, pp. 97-103.

Press, J. R., Shrestha, K. K., \& Sutton, D. A. (2000). Annotated checklist of the flowering plants of Nepal. Natural History Museum Publications.

Rajbhandari, K. R. (2016). History of botanical explorations in Nepal: 1802-2015. In: Frontiers of Botany, Jha, P. K., Siwakoti, M., \& Rajbhandary, S. (eds.), Central Department of Botany, Tribhuvan University, Kathmandu, pp. 1-99.

Rajbhandari, K. R., \& Adhikari, M. K. (2009). Endemic flowering plants of Nepal. Part 1. Kathmandu, Nepal: Department of Plant Resources.

Rajbhandari, K. R., \& Dhungana, S. K. (2010). Endemic flowering plants of Nepal. Part 2.
Kathmandu, Nepal: Department of Plant Resources.

Rajbhandari, K. R., \& Dhungana, S. K. (2011). Endemic flowering plants of Nepal. Part 3. Kathmandu, Nepal: Department of Plant Resources.

Rajbhandari, K. R. (1976). History of botanical explorations in Nepal. Journal of the Bombay Natural History Society, 73(1), 468-481.

Rajbhandari, K.R., Rai, S. K., Bhatt, G. D., Chhetri, R., \& Khatri, S. (2017). Flowering Plants of Nepal: An Introduction. Department of Plant Resources, Kathmandu, Nepal, 432p.

Rajbhandary, S. (2016). Fern and fern allies of Nepal. Frontiers of Botany, 124-150.

Rajbhandary, S., \& Winkler, D. (2015). Ethnobotany. In: Nepal: An Introduction to the Natural History, Ecology and Human Environment of the Himalayas, Miehe, G., Pendry, C. A., \& Chaudhary, R. (eds.), Edinburgh: Royal Botanic Garden Edinburgh. pp. 271-285.

Rajbhandary, S., Siwakoti, M., Rai, S. k, \& Jha, P. K. (2020). An Overview of Plant Diversity in Nepal. Plant Diversity in Nepal, Botanical Society of Nepal, Kathmandu, 1-15.

Raskoti, B. B. (2009). The Orchids of Nepal. Kathmandu, Nepal: Quality Printers.

Ribeiro, S., Fernandes, J. P., \& Espírito-Santo, M. D. (2014). Diversity and floristic patterns of mediterranean grasslands: the relative influence of environmental and land management factors. Biodiversity and conservation, 23(12), 2903-2921. doi: https:// doi.org/10.1007/s10531-014-0754-y

Rokaya, M. B., Raskoti, B. B., Timsina, B., \& Münzbergová, Z. (2013). An annotated checklist of the orchids of Nepal. Nordic Journal of Botany, 31(5), 511-550. doi: https:// doi.org/10.1111/j.1756-1051.2013.01230.x

Sahu, S. C., \& Dhal, N. K. (2012). Floristic composition, diversity and status of Threatened medicinal plants in tropical forests of Malyagiri Hill Ranges, Eastern Ghats, India. Tropical forests, 203-214.

Sakakibara, K. (2016). Technological innovations give rise to a new era of plant evolutionary developmental biology. Advances in botanical research, 78, 3-35. doi: https://doi. org/10.1016/bs.abr.2016.01.001

Shackleton, C.M. and D. Pullanikkatil. 2019. Considering the links between non-timber forest products and poverty alleviation. In Poverty Reduction Through Non-Timber Forest Products, pp.15-28, Springer, Cham. 
doi: $\quad$ https://doi.org/10.1007/978-3-31975580-9_2

Shrestha, B. B. (2019). Management of Invasive Alien Plants in Nepal: Current Practices and Future Prospects. In Tropical Ecosystems: Structure, Functions and Challenges in the Face of Global Change, pp. 45-68. Springer, Singapore. doi: https://doi.org/10.1007/978981-13-8249-9_4

Shrestha, K. (1998). Dictionary of Nepalese plant names. Kathmandu, Nepal: Mandala Book Point.

Shrestha, K. K. (2011). Plants, People and Environment: a web of life in the Himalayas. TU Bulletin Special Issue, 12, 57-67.

Shrestha, K. K., \& Bajracharya, S. B. (2019). Biodiversity in Nepal. In: Global Biodversity, Vol 1 (Selected countries in Asia), Pullaiah, T. (ed.). Canada and USA: Apple Academic Press Inc., pp. 427-472.

Shrestha, K. K., Bhattarai, S., \& Bhandari, P. (2018). Handbook of Flowering Plants of Nepal (Vol. 1 Gymnosperms and Angiosperms: CycadaceaeBetulaceae). Scientific Publishers.

Søchting, U. (2015). Lichens. In: Nepal: An Introduction to the Natural History, Ecology and Human Environment of the Himalayas, Miehe, G., Pendry, C. A., \& Chaudhary, R. (eds.), Edinburgh: Royal Botanic Garden Edinburgh. pp. 191-199.

Thapa, N., \& Maharjan, M. (2014). Invasive alien species: Threats and challenges for biodiversity conservation-A case study of Annapurna Conservation Area, Nepal. Proceedings of the Internatioonal Conference on Invasive Alien Species Management.

Vallès, J., Canela, M. A., Garcia, S., Hidalgo, O., Pellicer, J., Sánchez-Jiménez, I., SiljakYakovlev, S., Vitales, D., Garnatje, T. (2013). Genome size variation and evolution in the family Asteraceae. Caryologia: International Journal of Cytology, Cytosystematics and Cytogenetics, 66(3), 221-235. doi: https://doi.org/10.1080/ 00087114.2013.829690

Vitousek, P. M., D'antonio, C. M., Loope, L. L., Rejmanek, M., \& Westbrooks, R. (1997). Introduced species: a significant component of human-caused global change. New Zealand Journal of Ecology, 1-16. doi: https:// www.jstor.org/stable/24054520

Watson, L., \& Dallwitz, M. J. (1992). The families of flowering plants: descriptions, illustrations, identification, and information retrieval. (accessed on 3 June 2021)

Watson, M. F., Mkiyama, S., Ikeda, H., Pendry, C., Rajbhandari, K. R., \& Shrestha, K. K. (eds). (2011). Flora of Nepal, Vol. 3. Edinburgh, UK: The Royal Botanic Garden.

World Conservation Monitoring Centre. (1992). Global biodiversity: Status of the Earth's Living Resources. Chapman and Hall. London. 


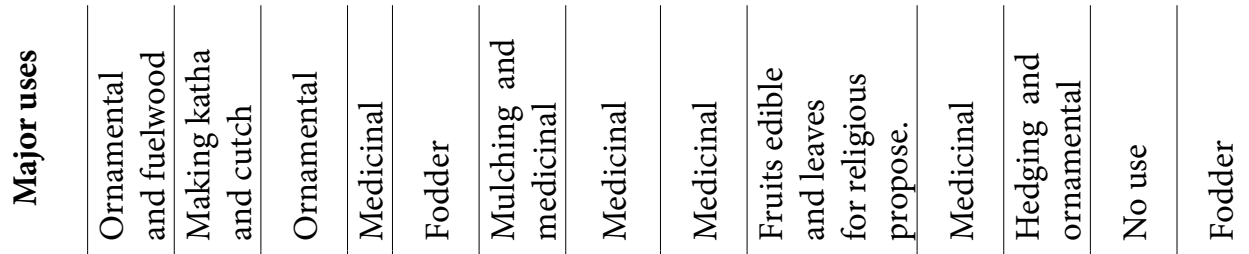

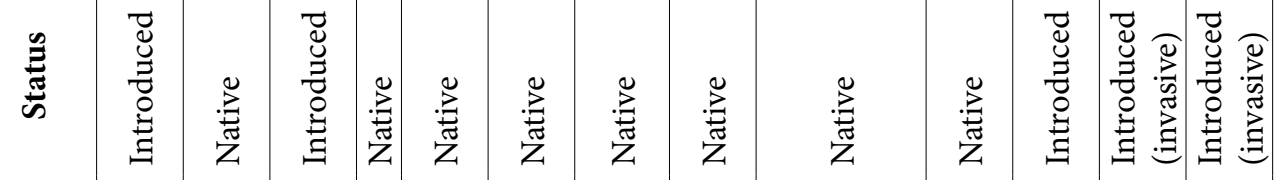

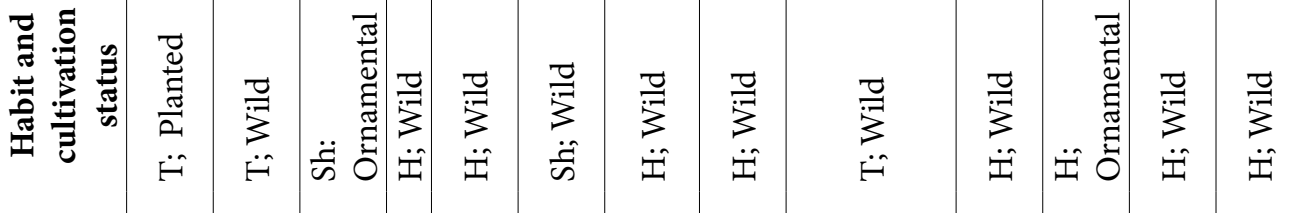

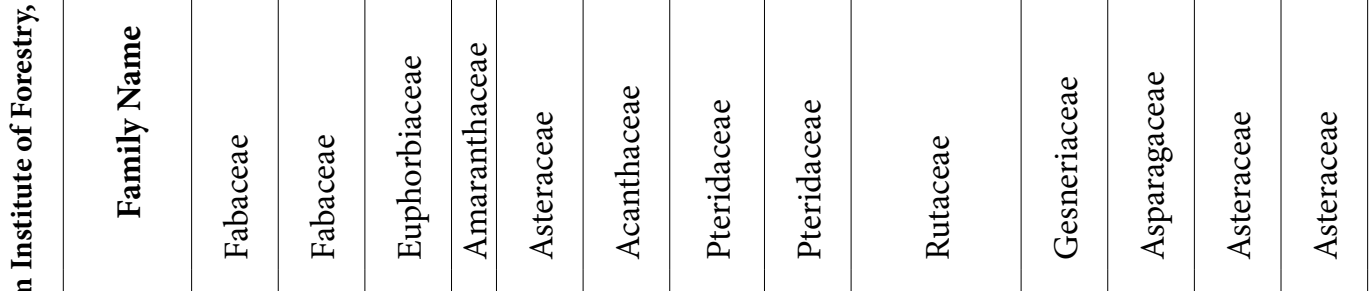

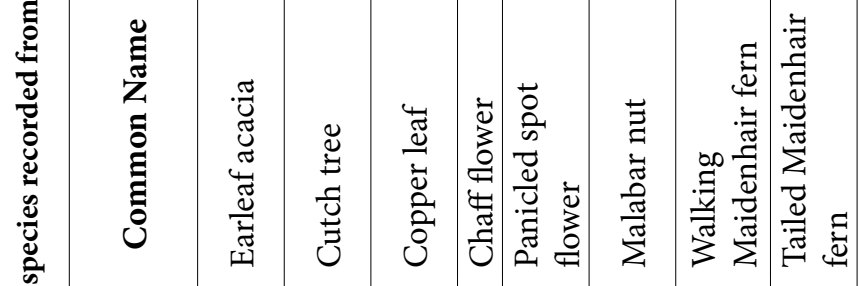

产

苞农

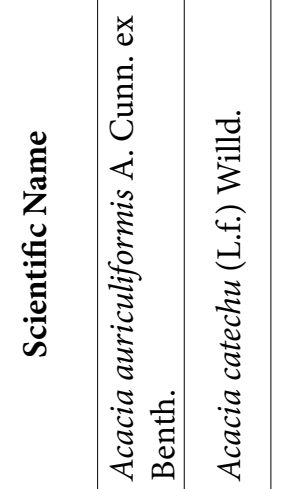

\begin{tabular}{l|l|l|l|l|l|}
$\dot{z}$ & - & $\sim$
\end{tabular}

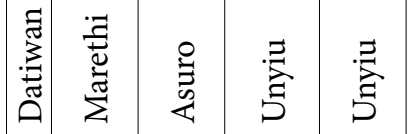

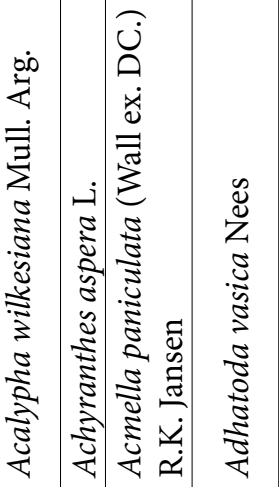

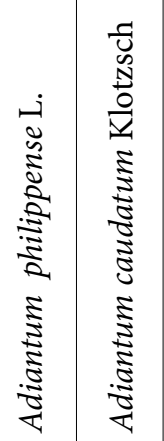

$\wedge \infty$ $\frac{0}{2}$
है
$\stackrel{0}{0}$
के

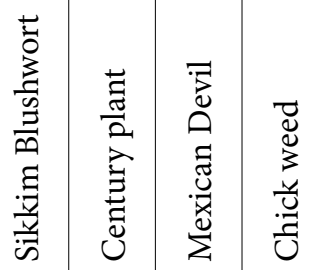

芦芦

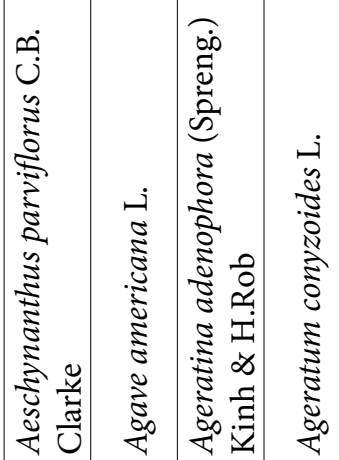

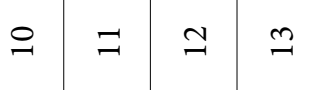




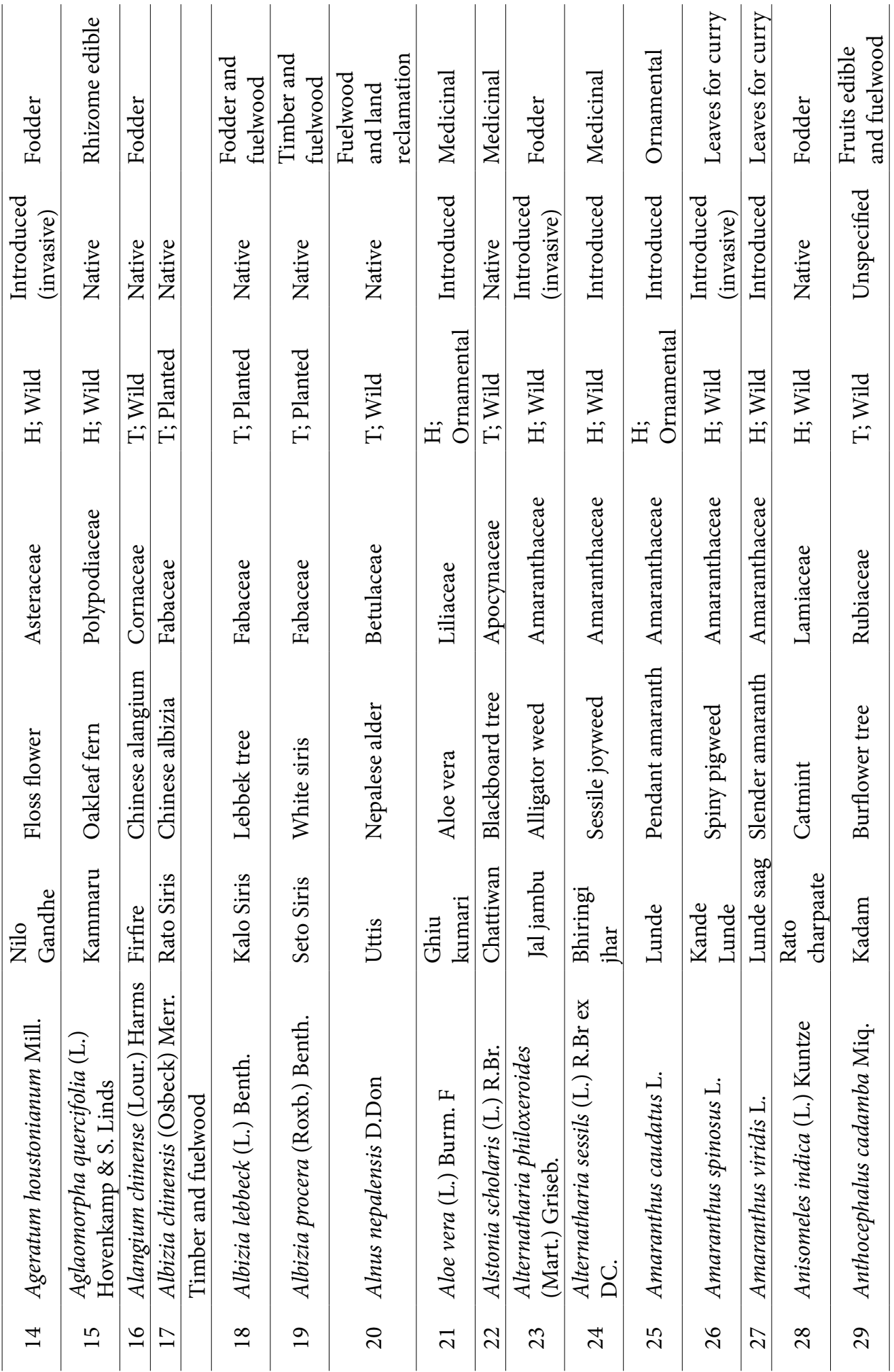




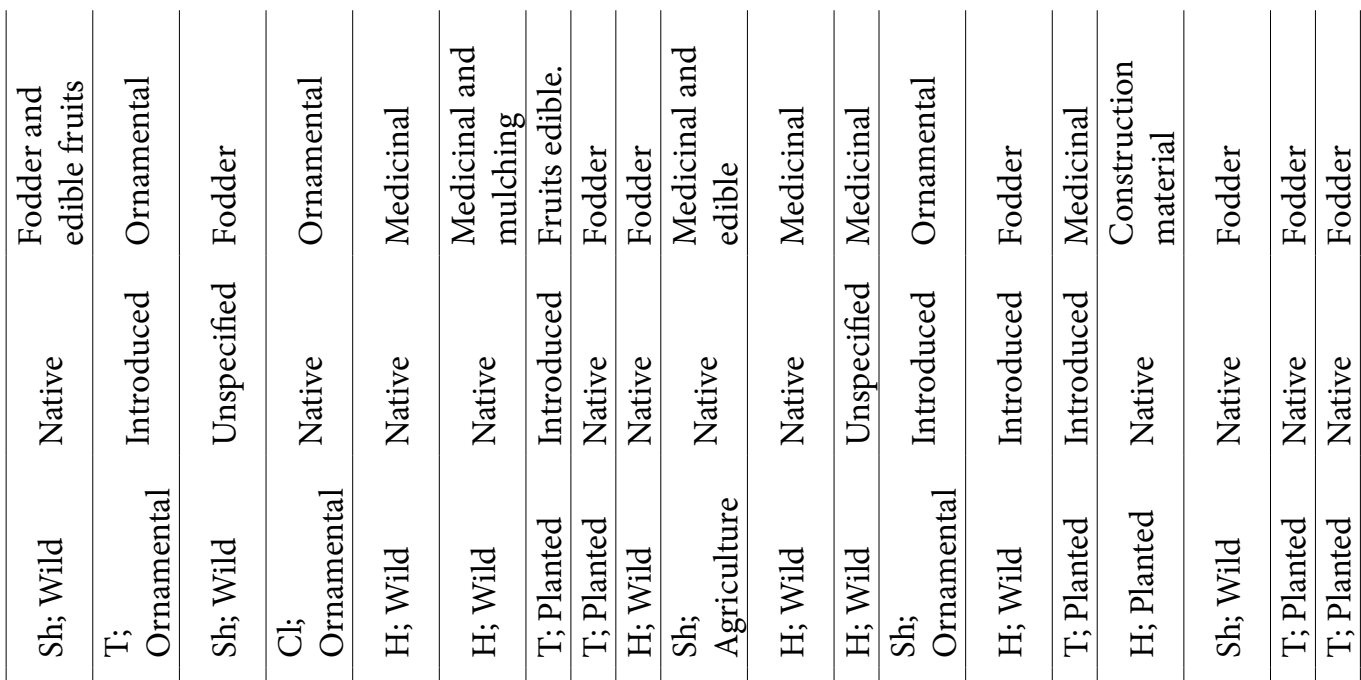

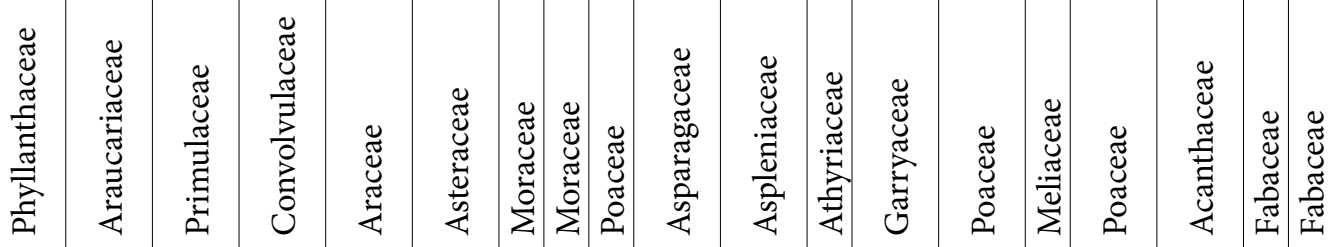

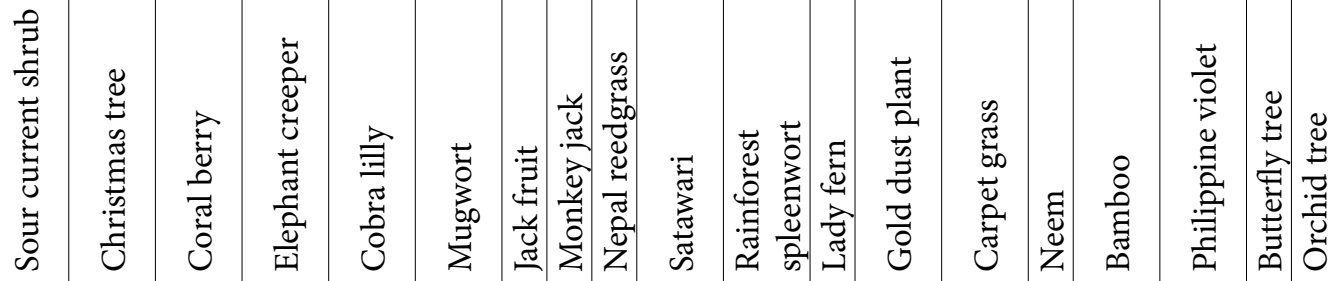

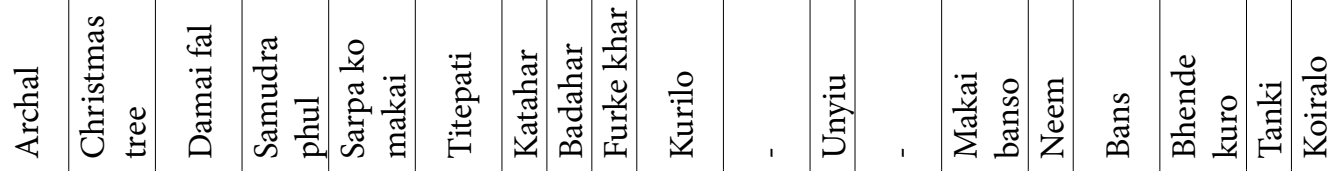

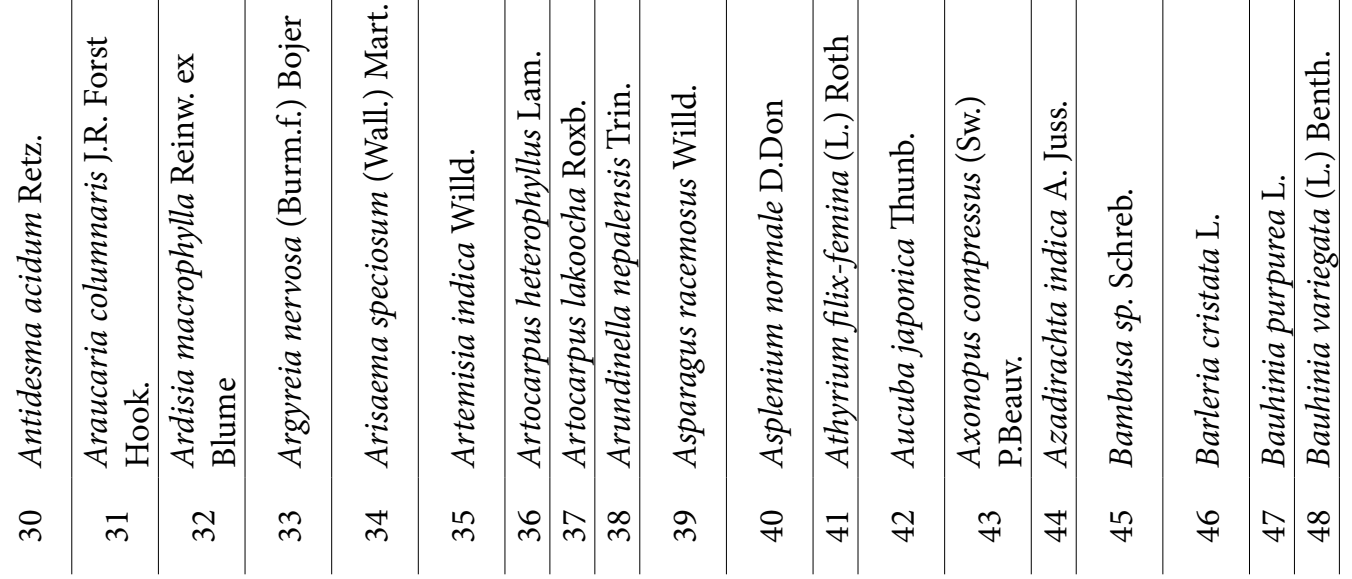




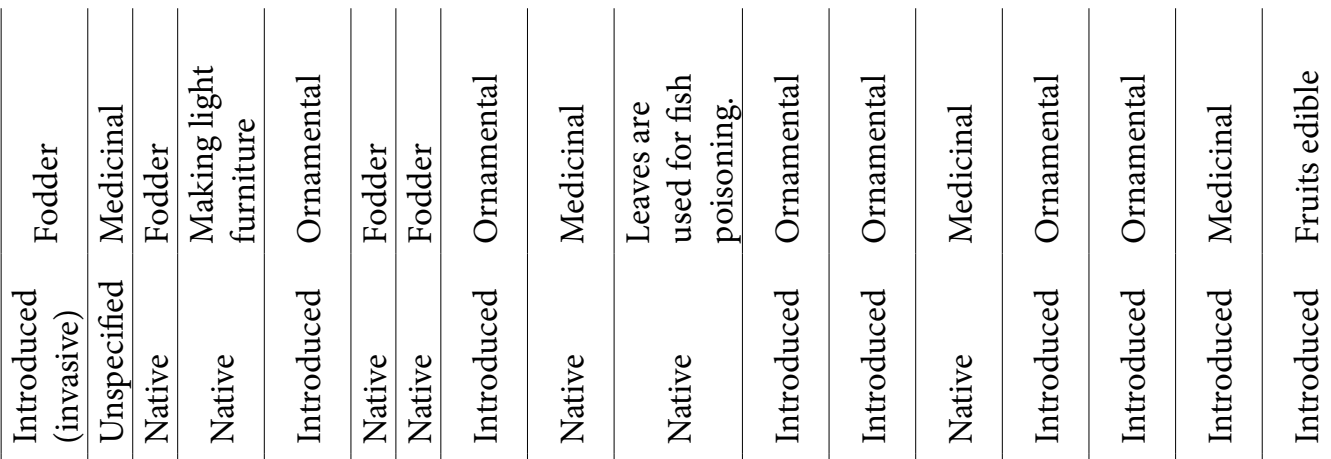

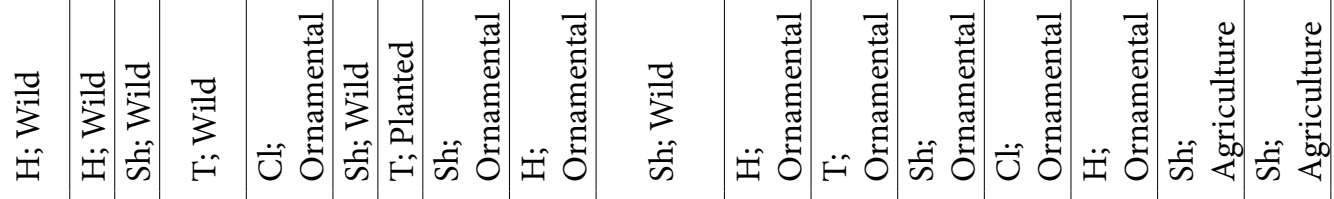

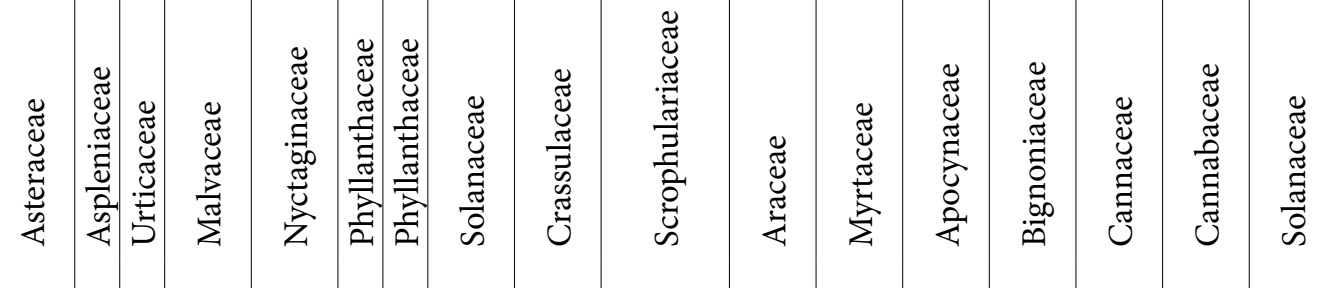

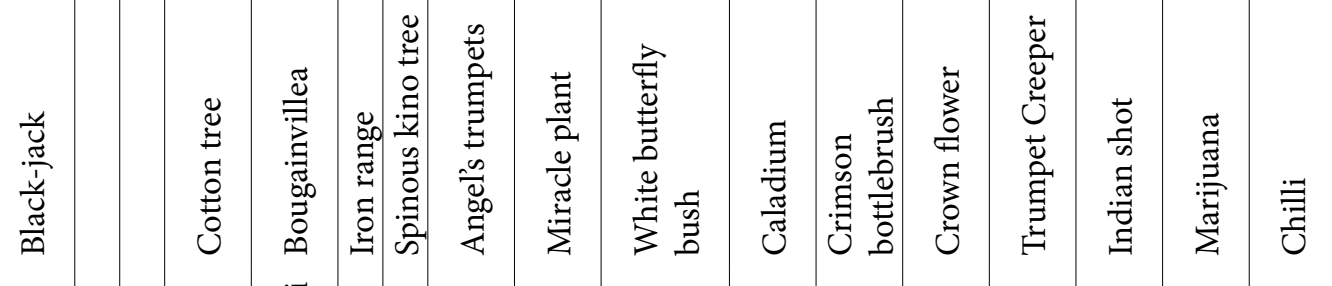

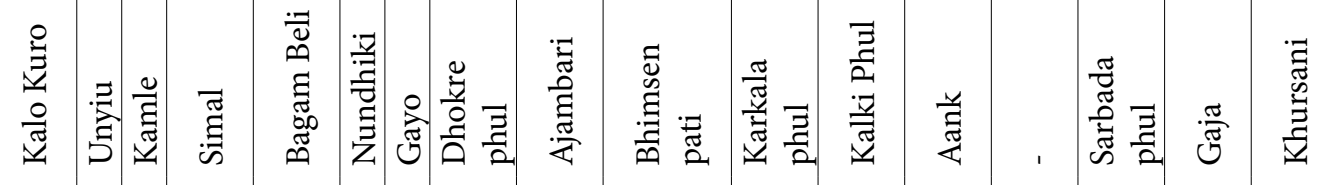

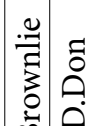
(1)

: - के

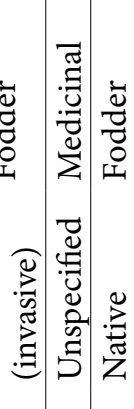
है कूष

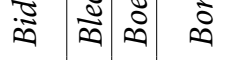

of की in in

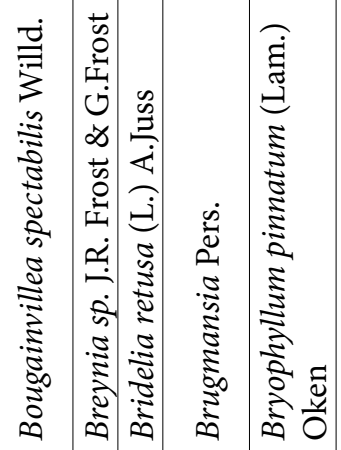

in 政织 in in

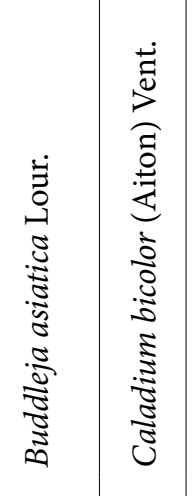

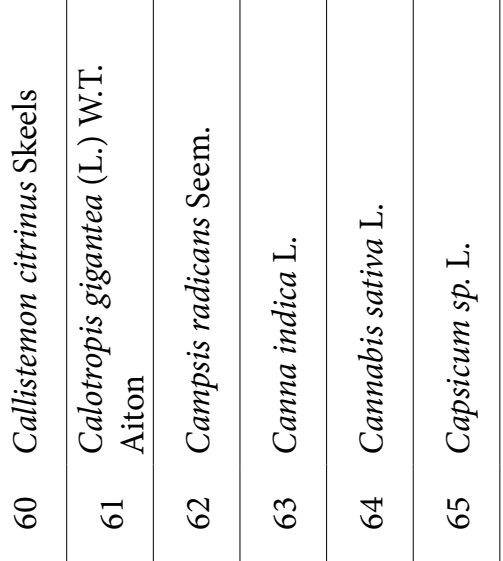




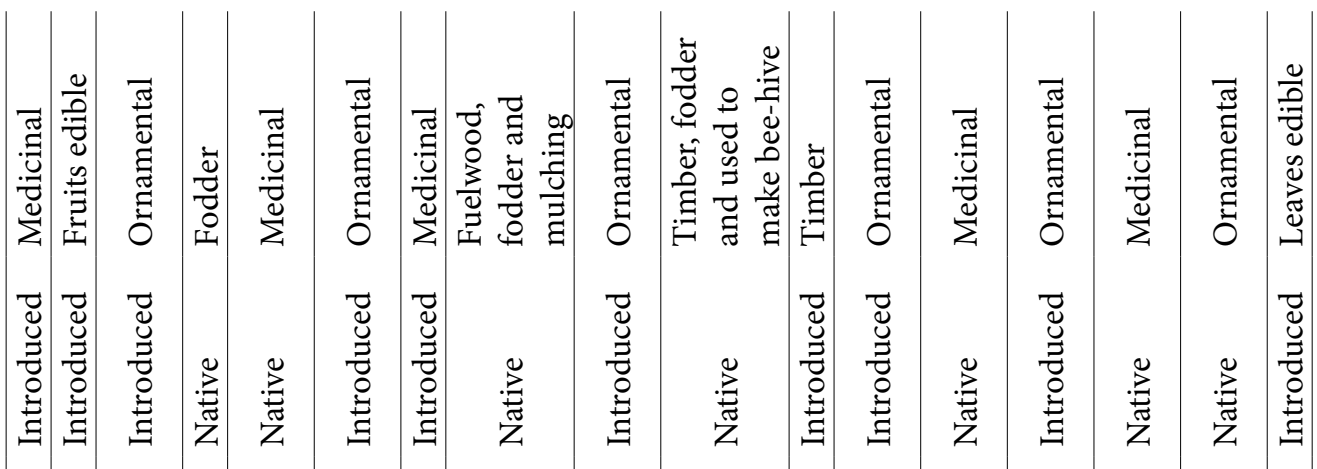

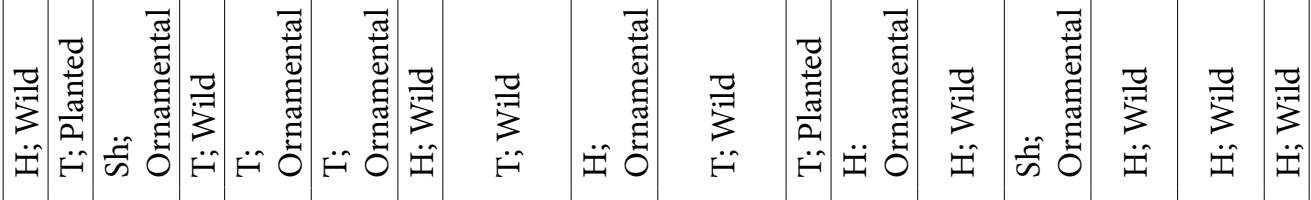

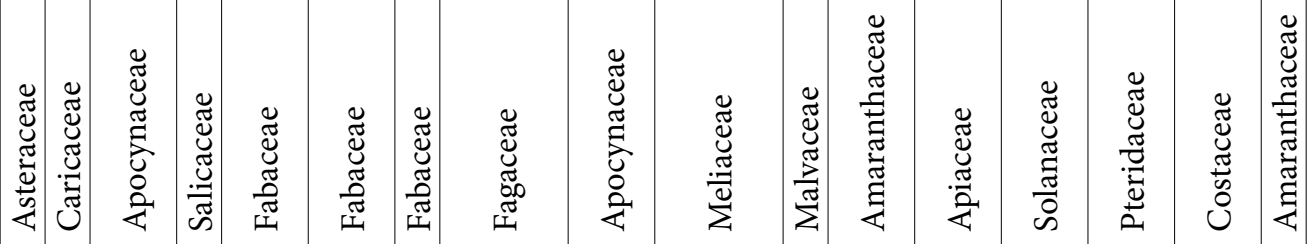

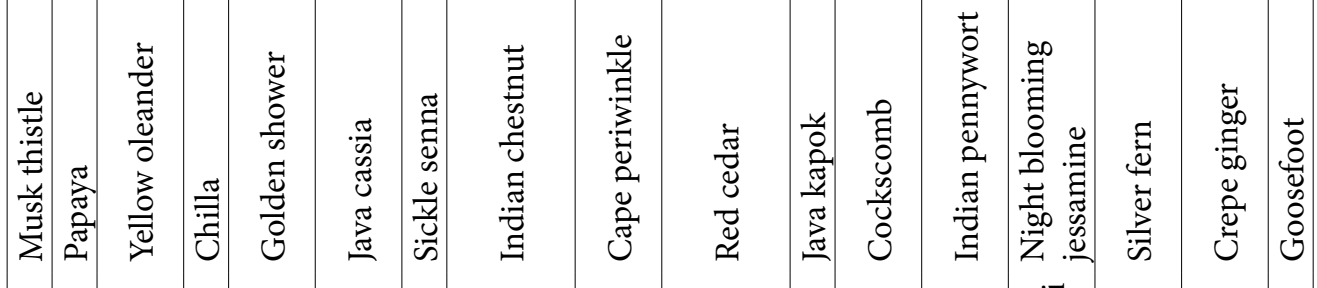

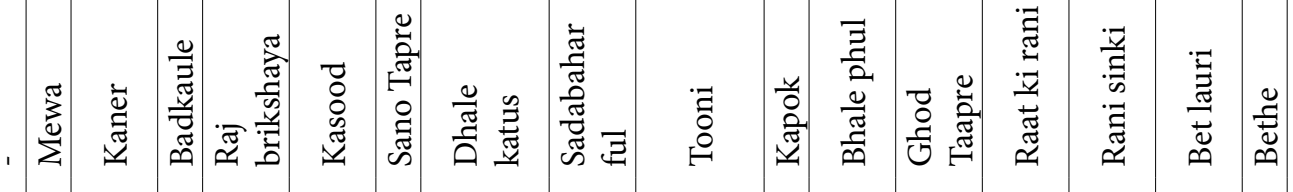

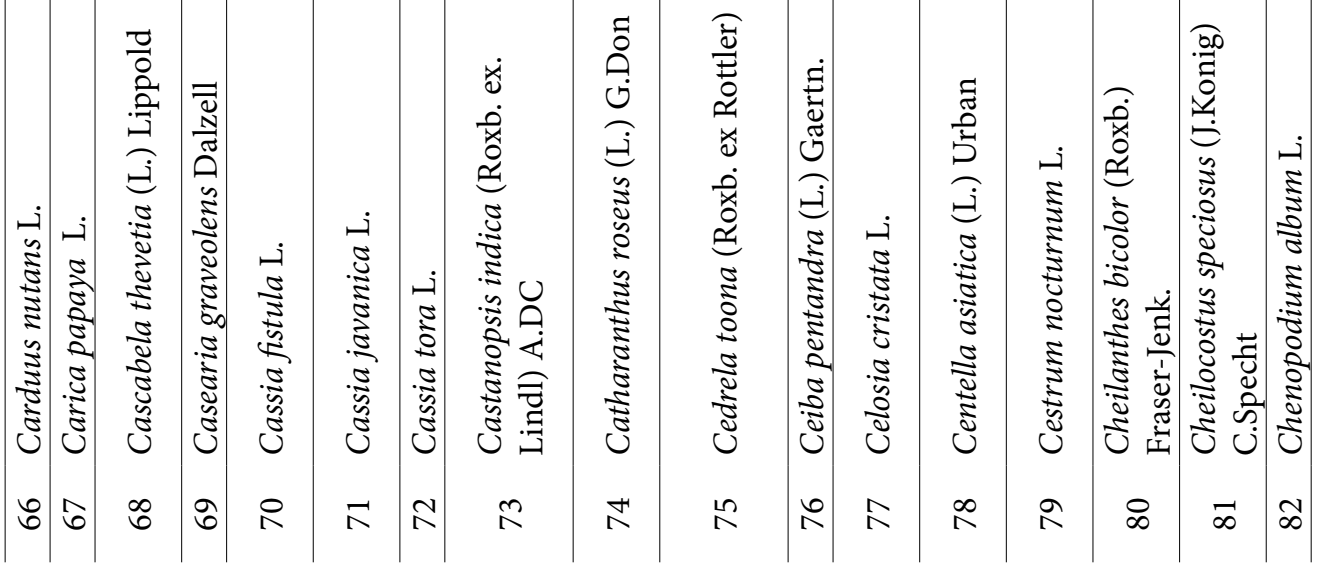




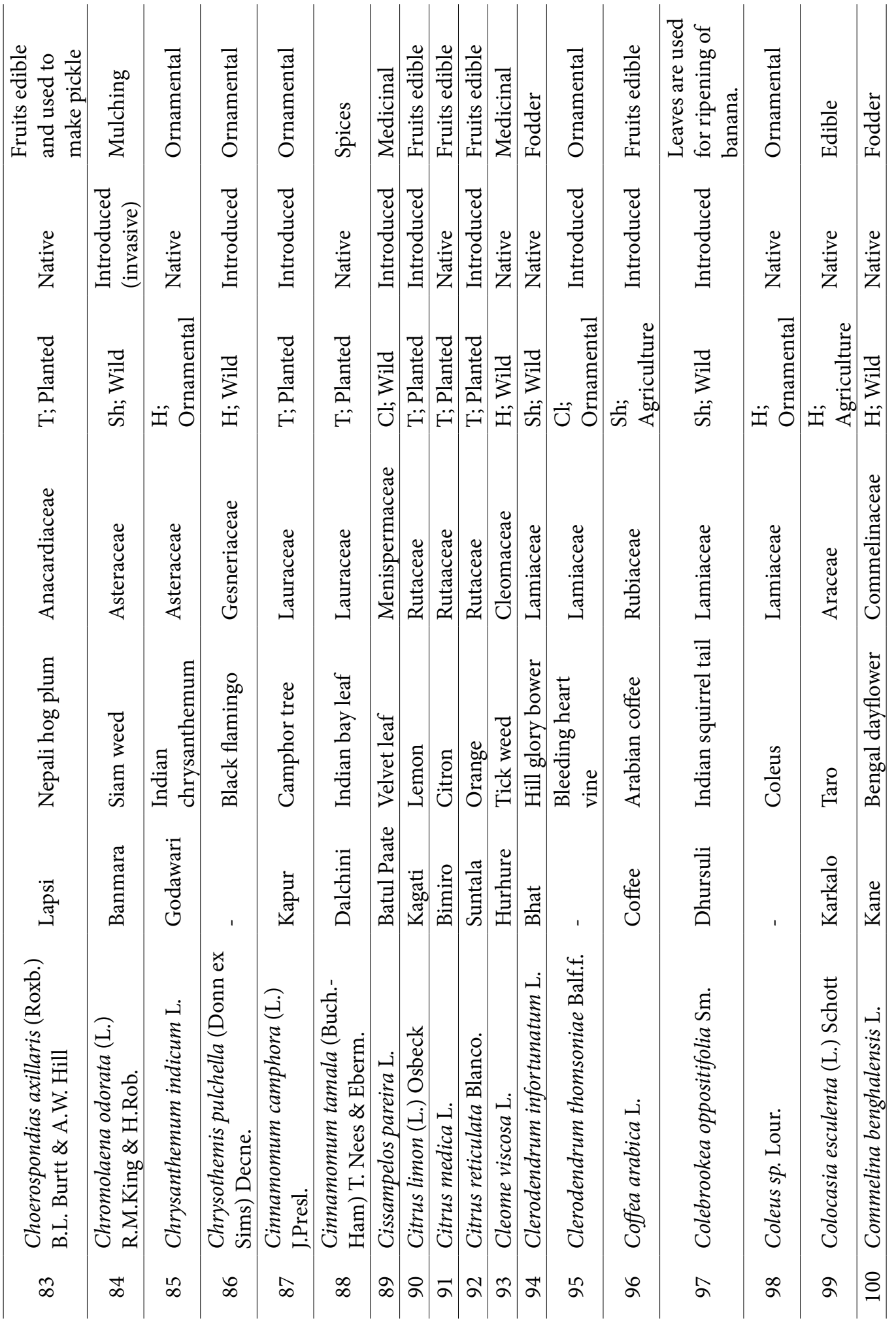




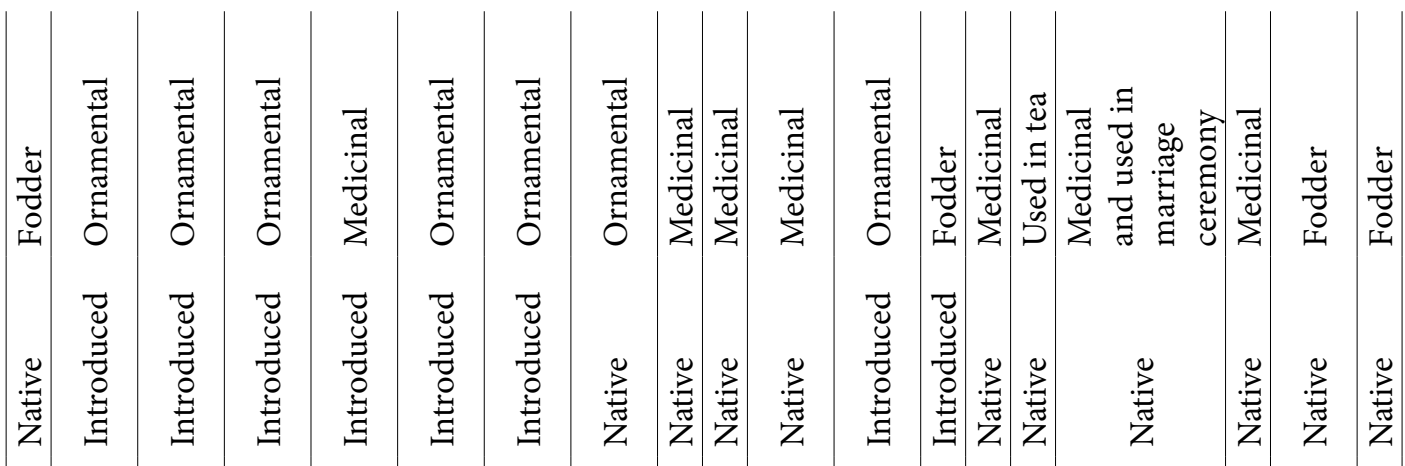

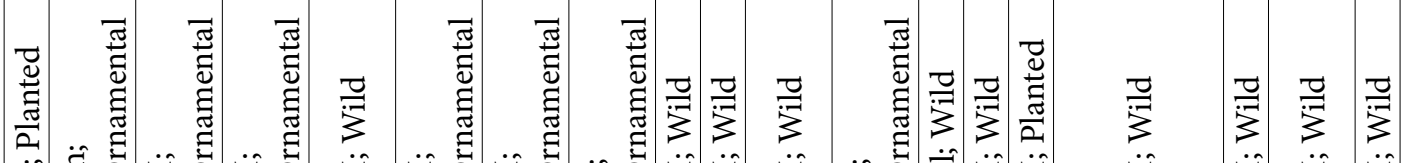
華

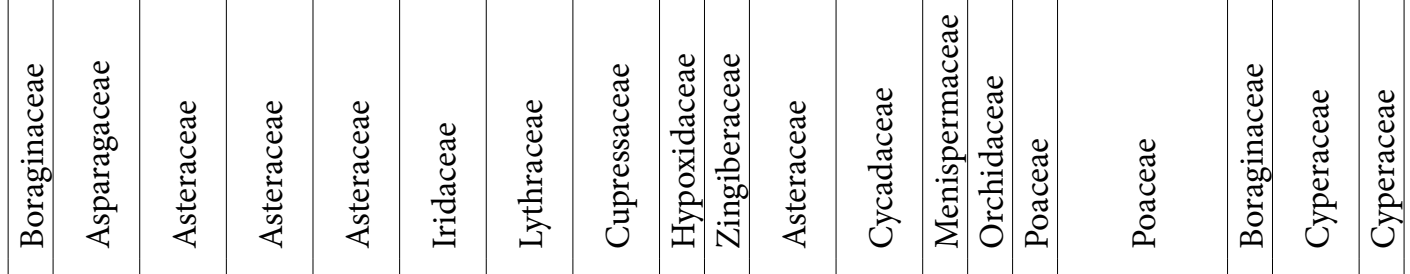

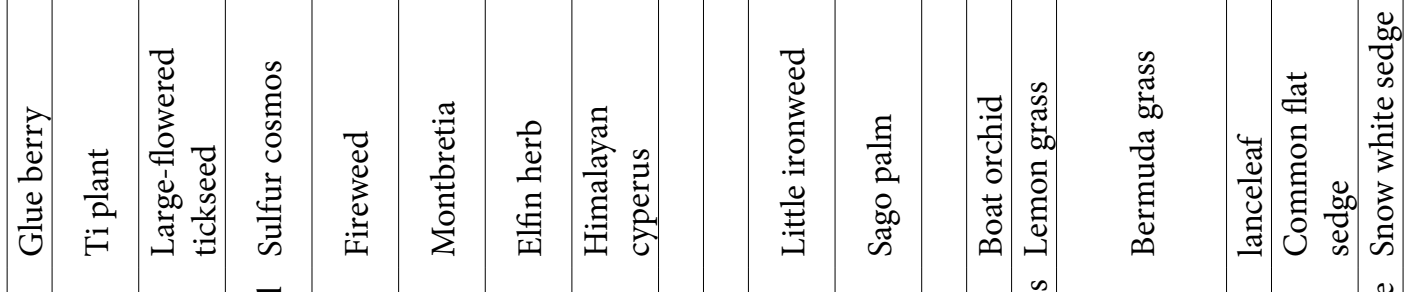

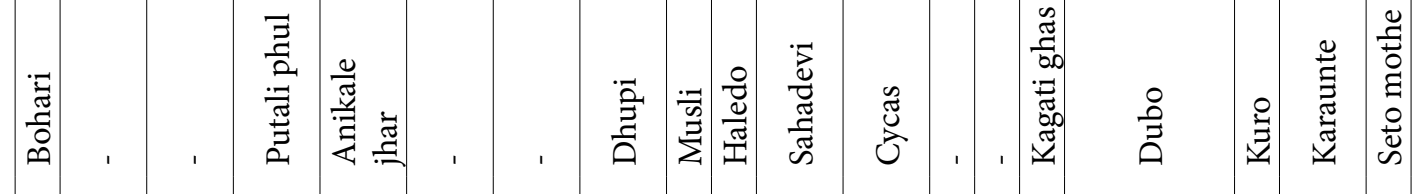

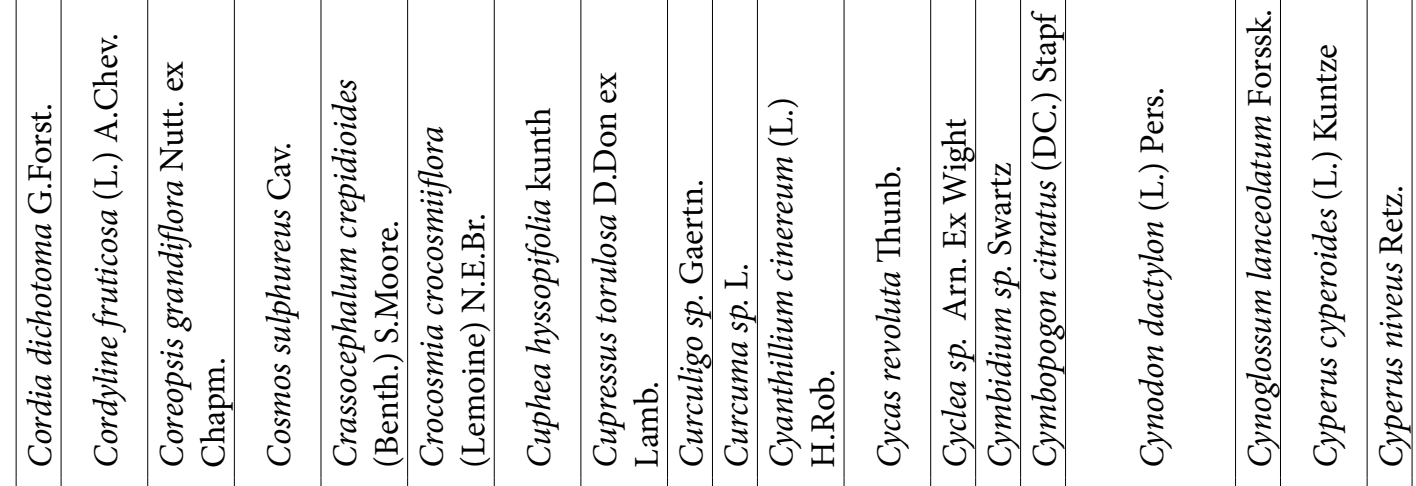

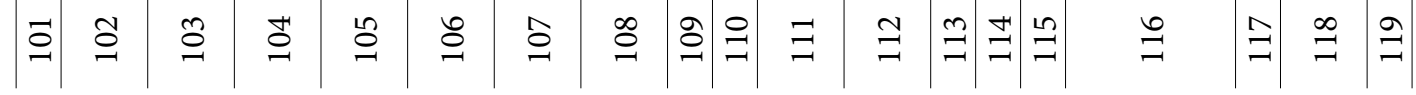




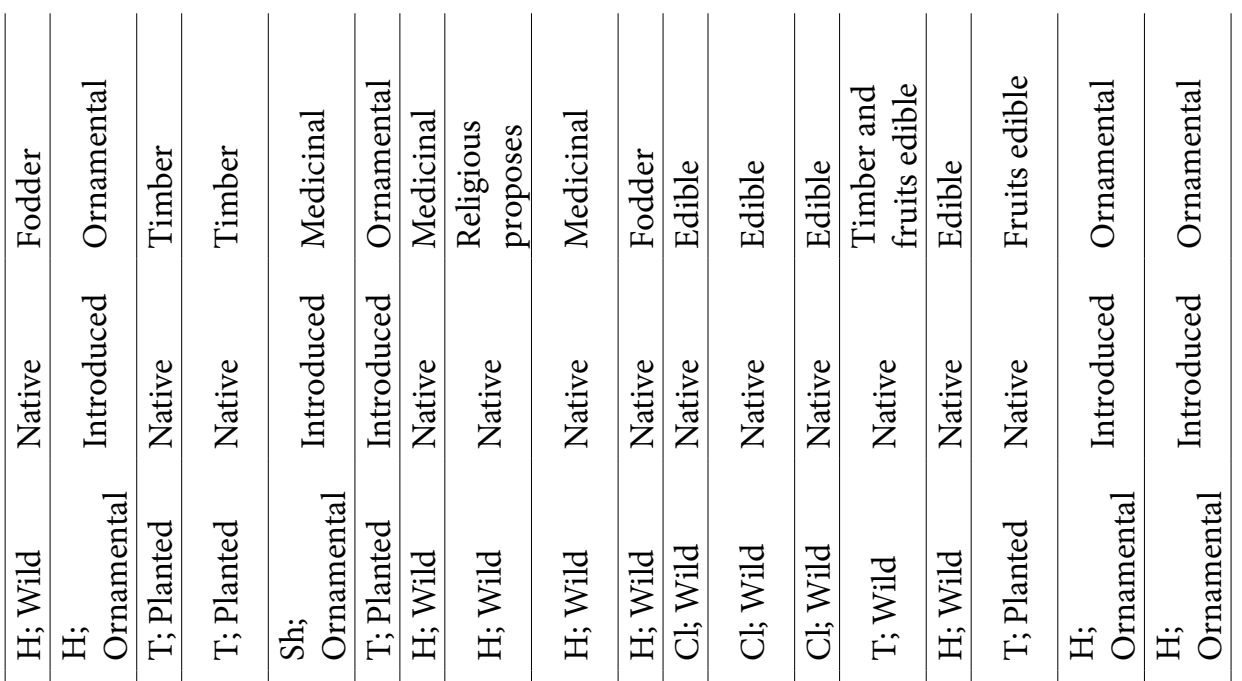

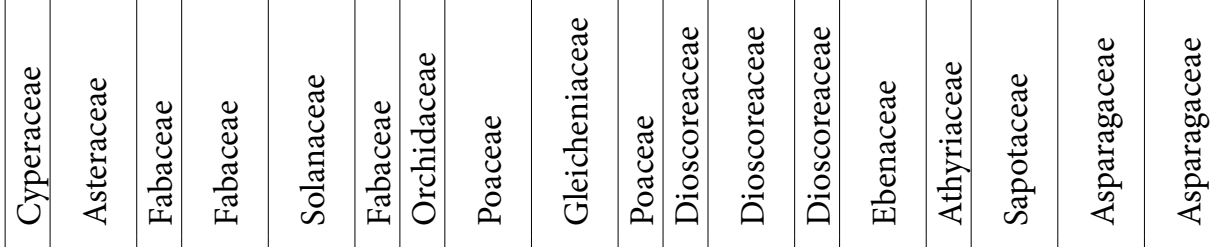

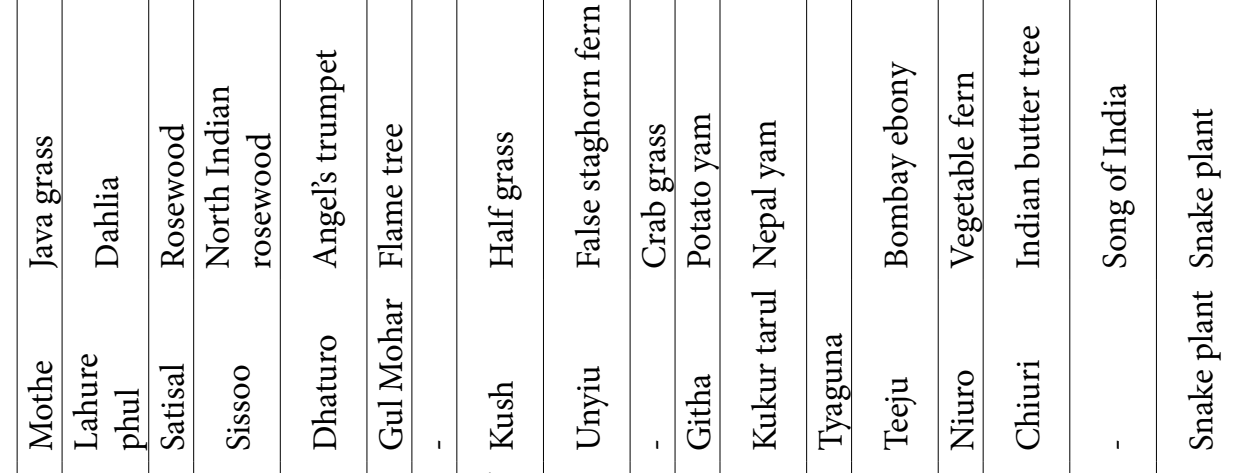

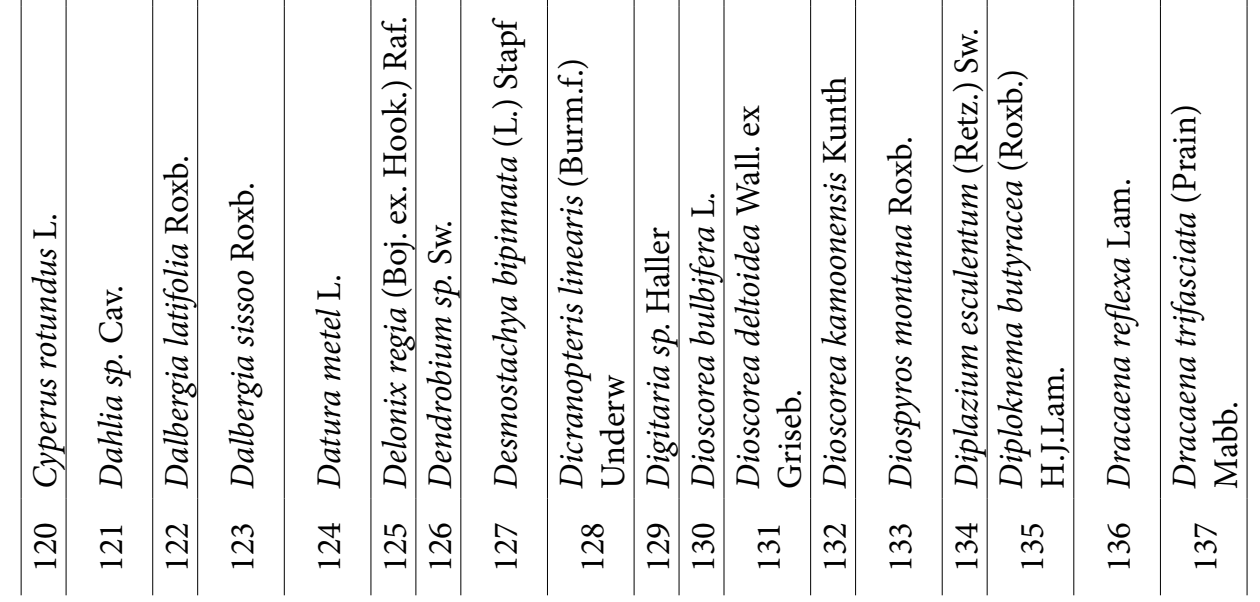




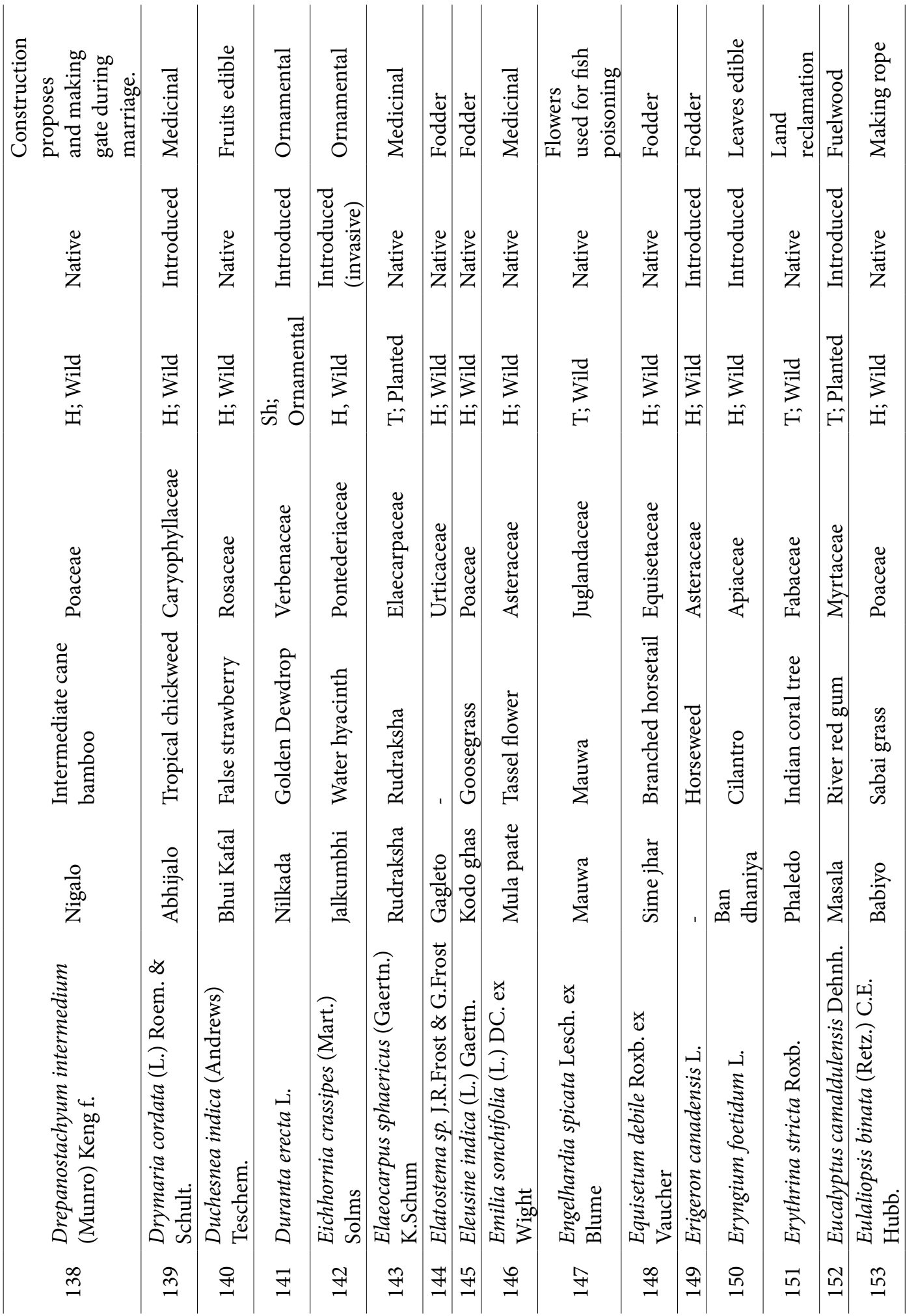




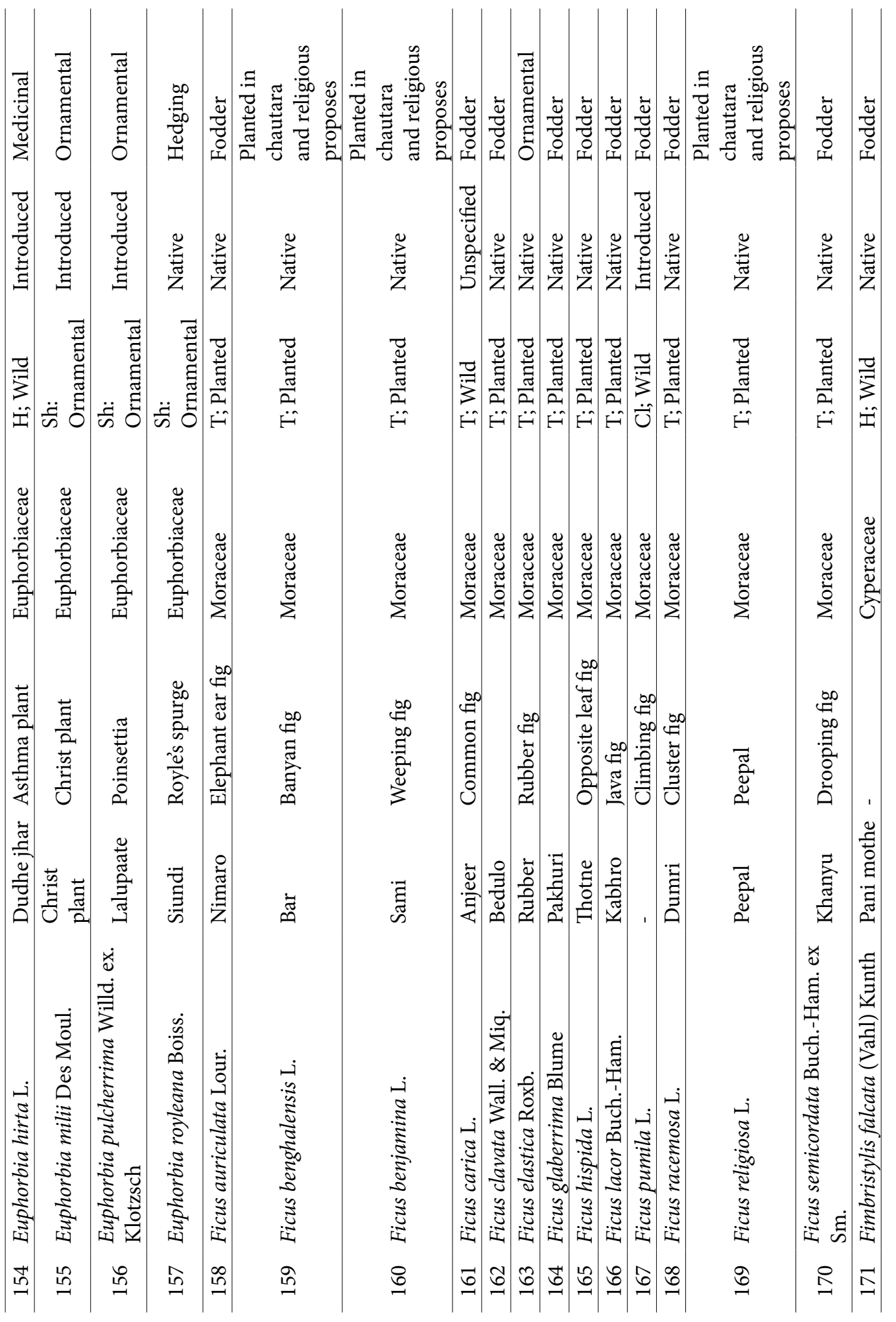




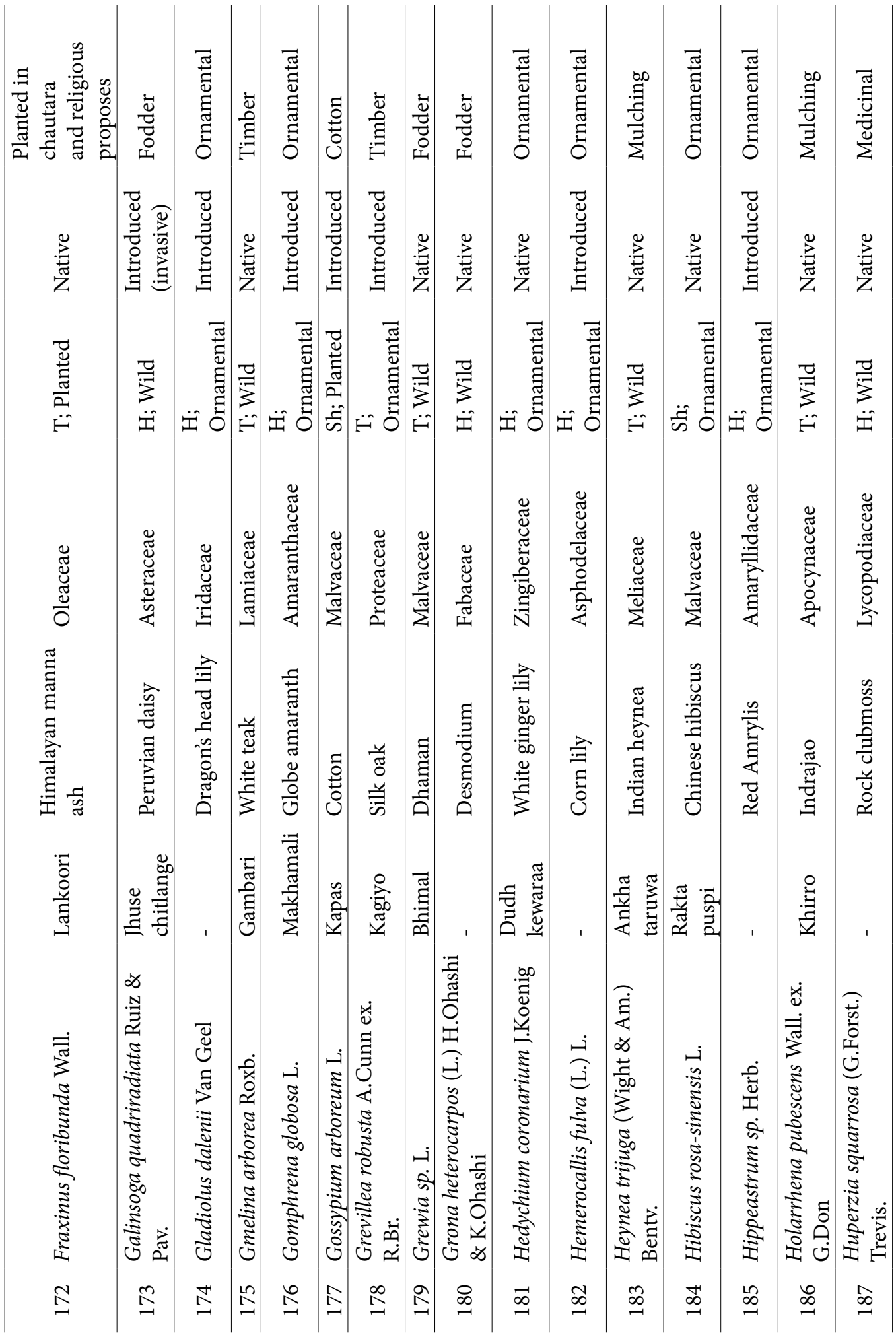




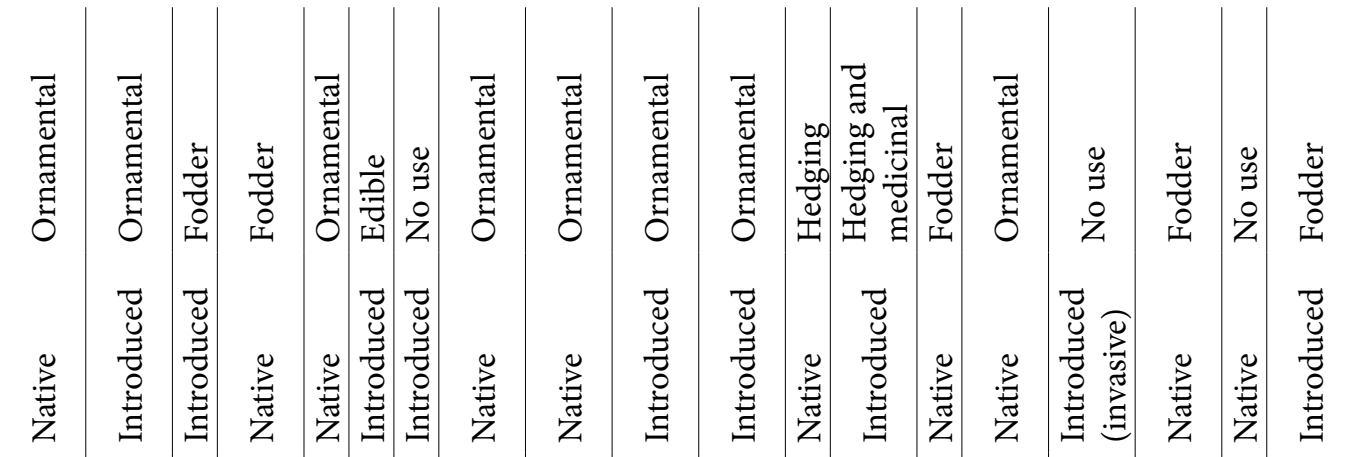

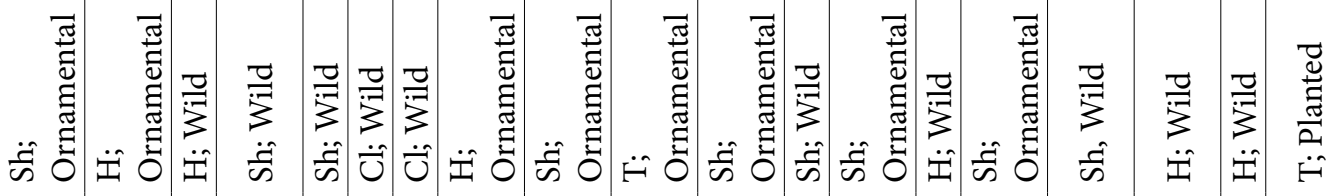

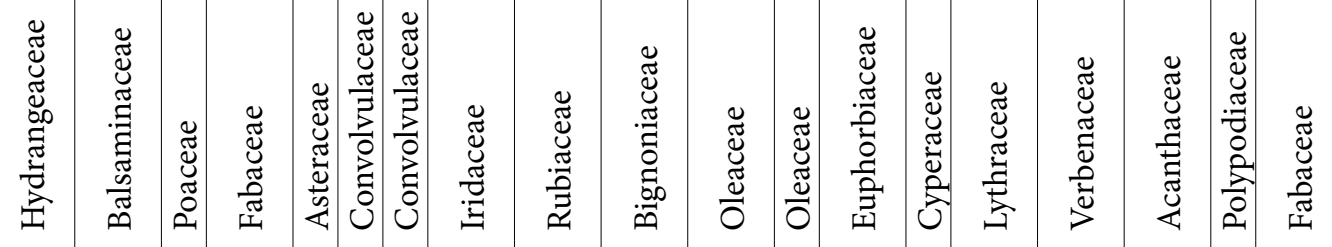

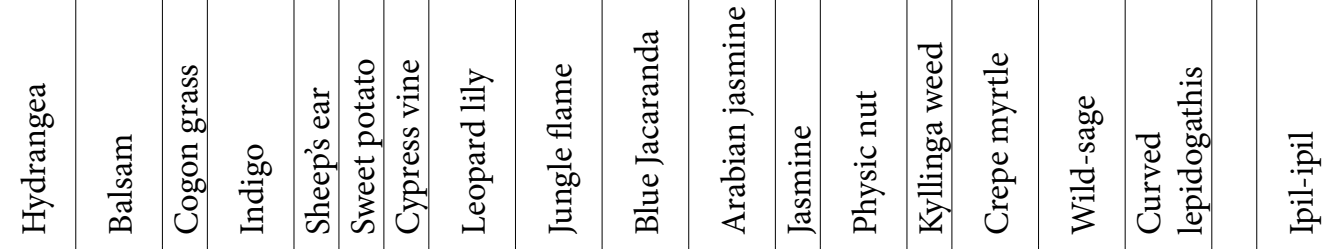

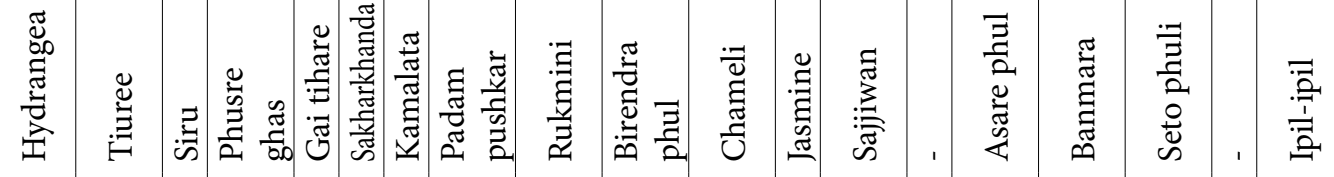

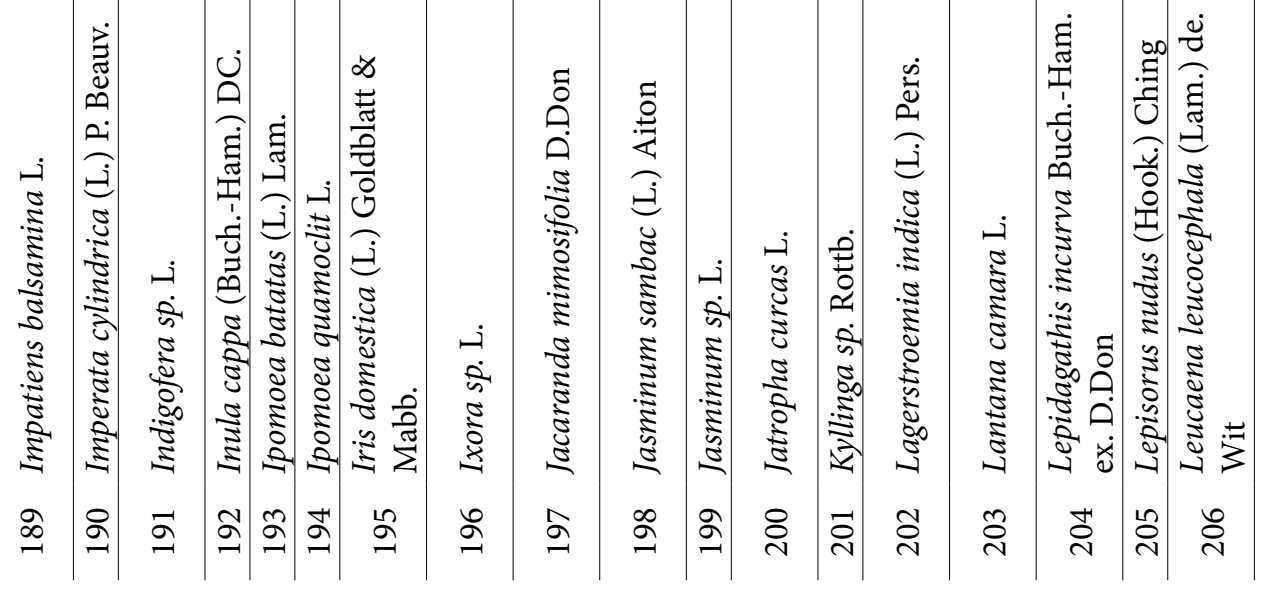




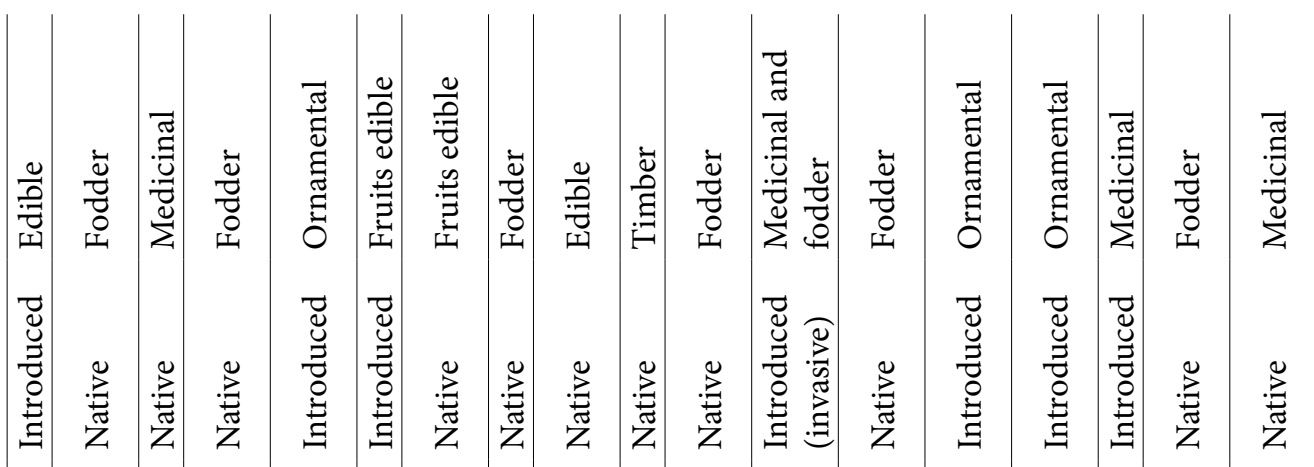

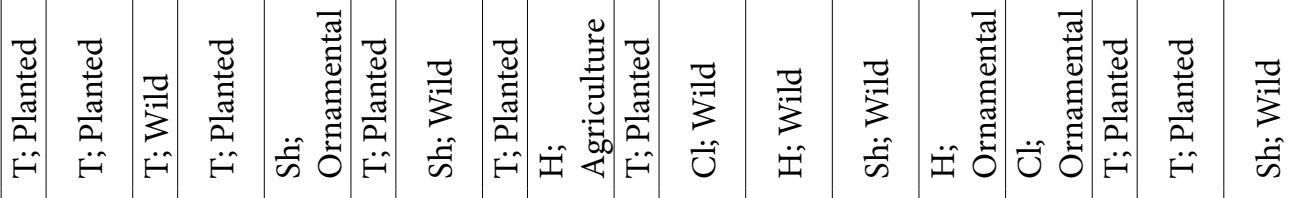

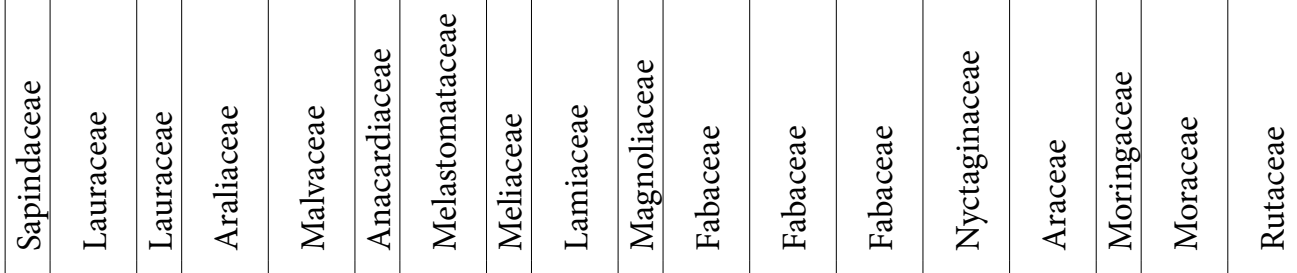

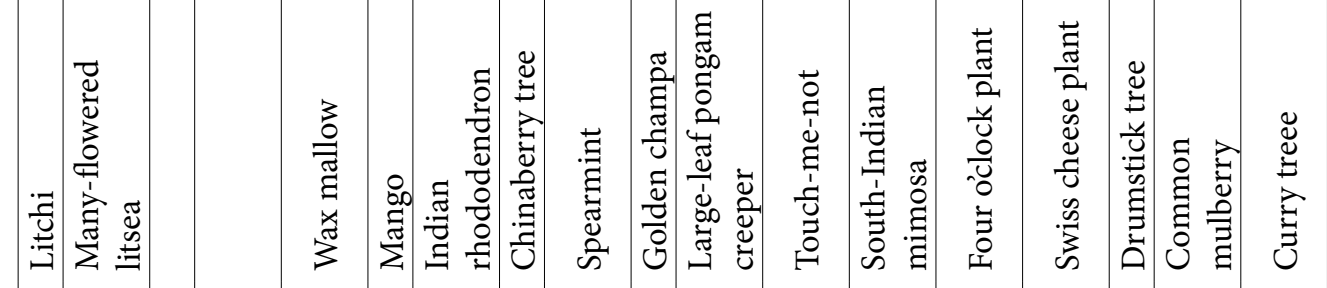

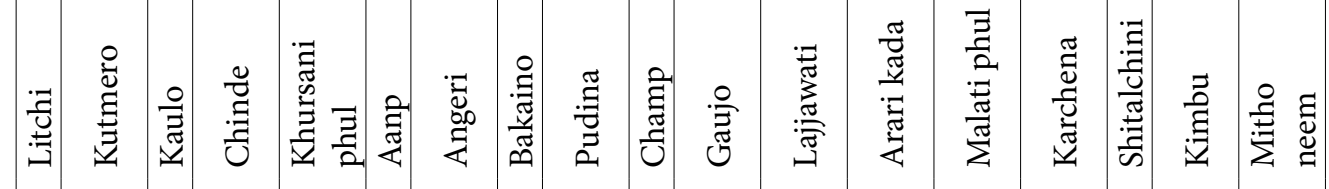

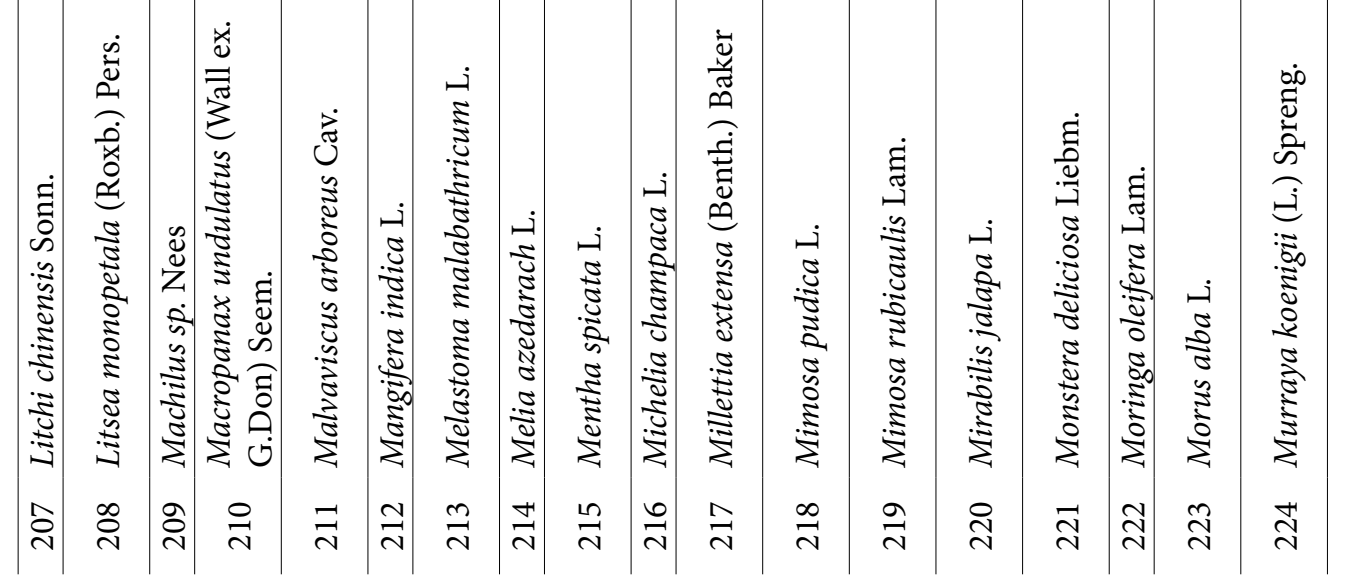




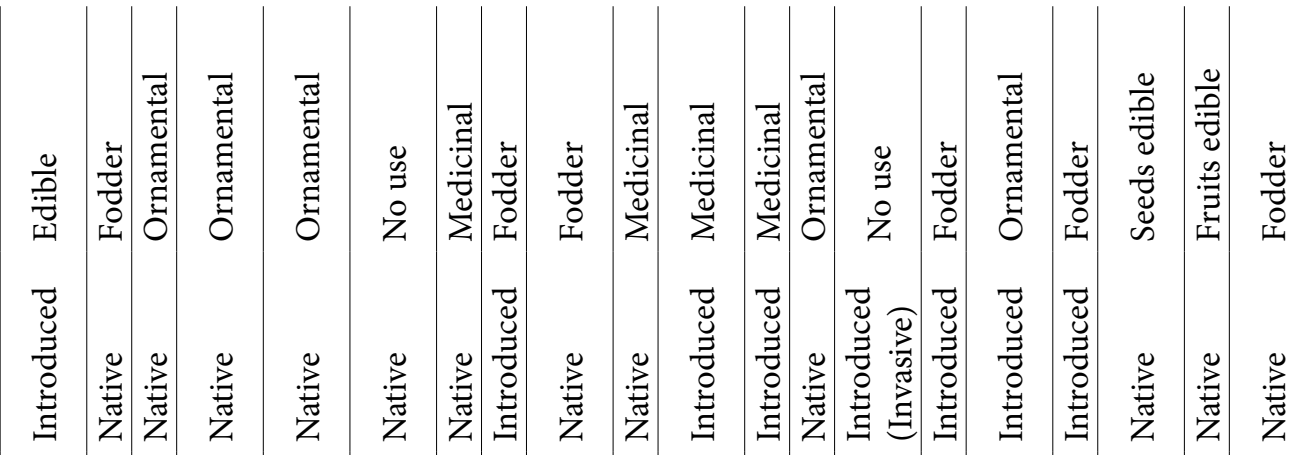

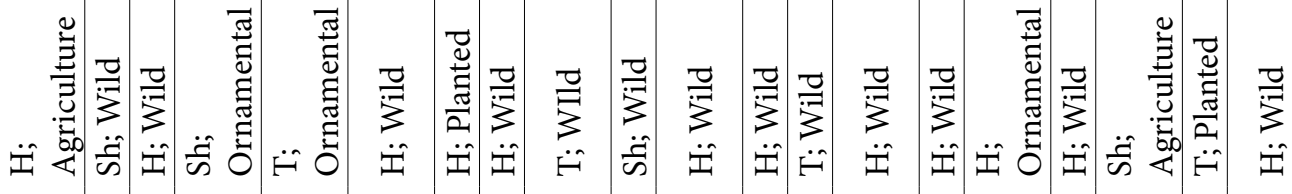

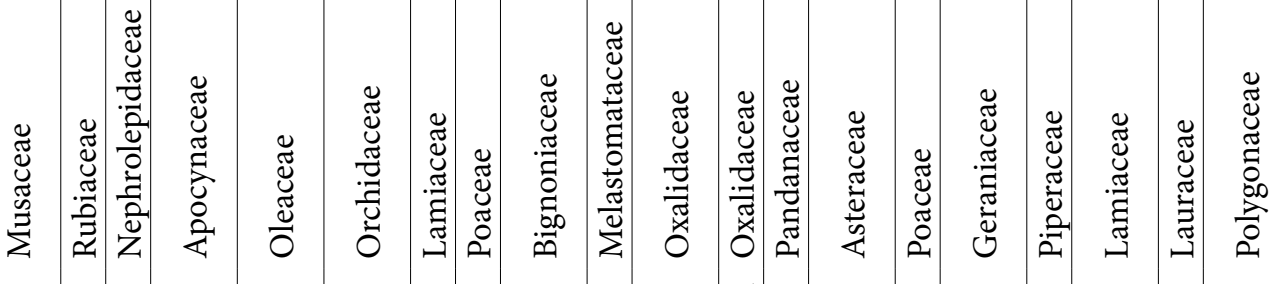

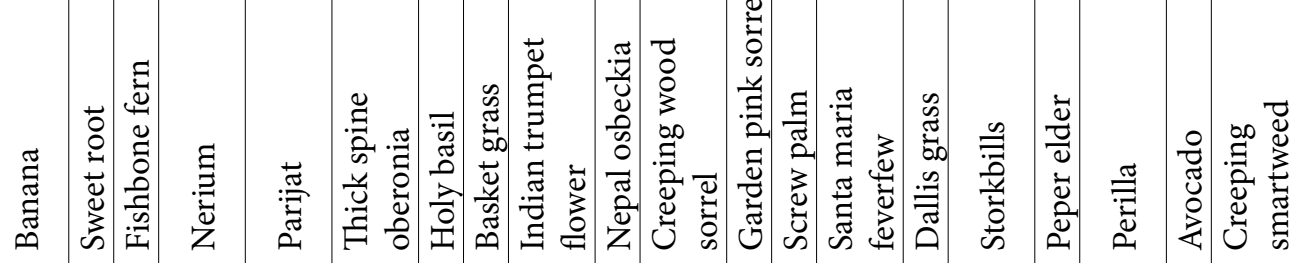

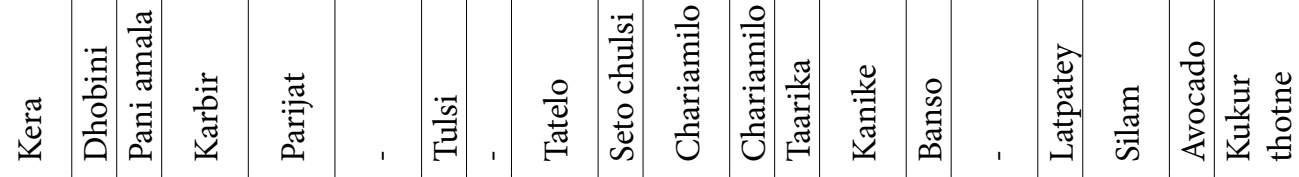

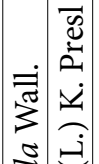
宏 $\quad$ 话 苟 


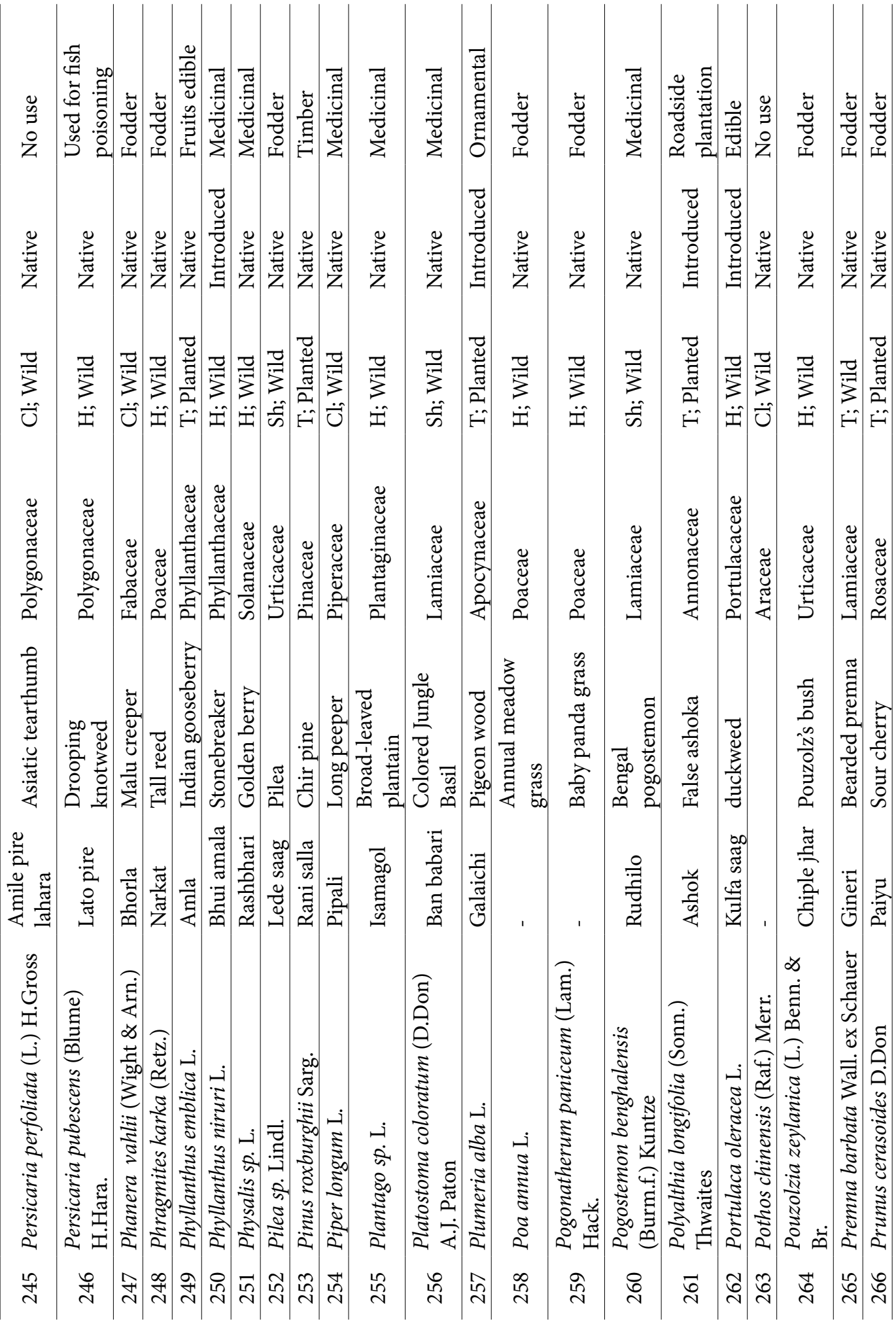




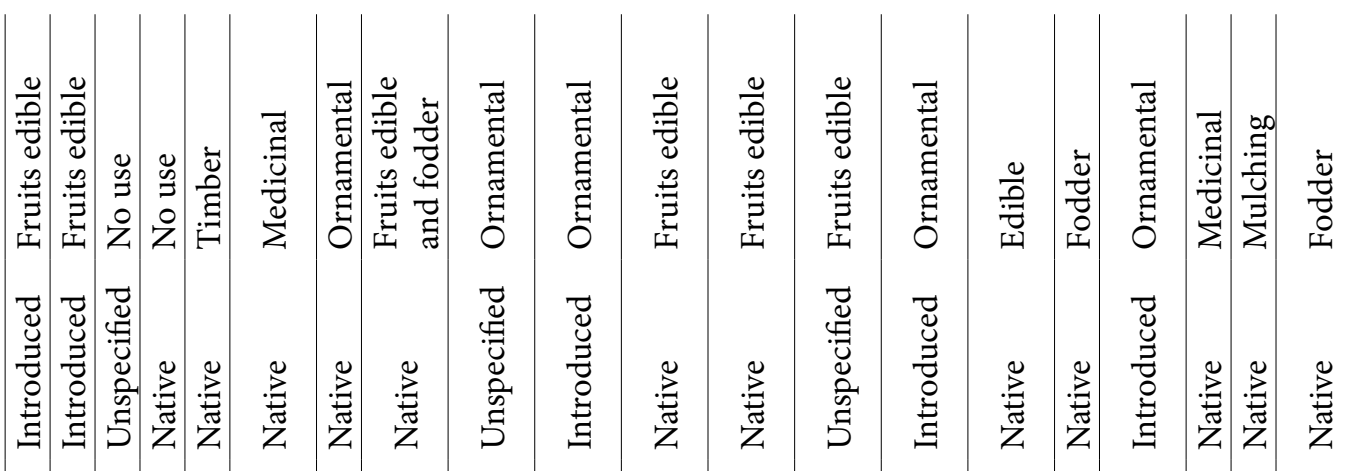

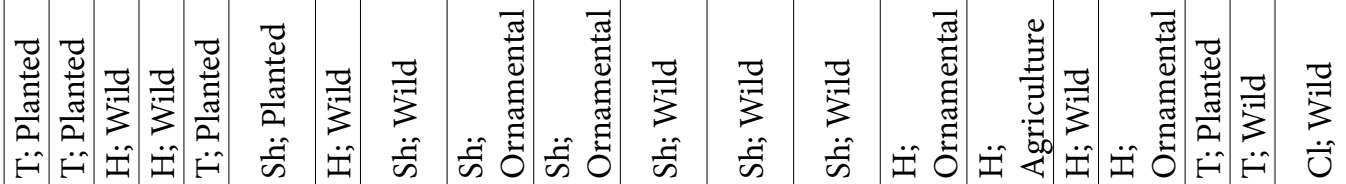

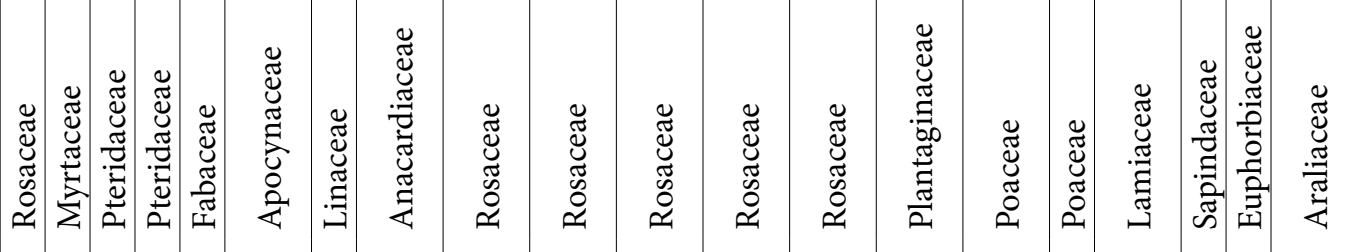

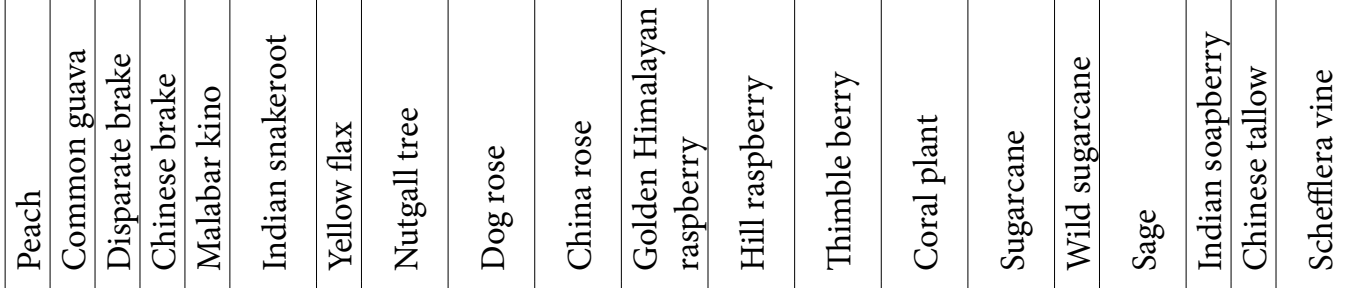

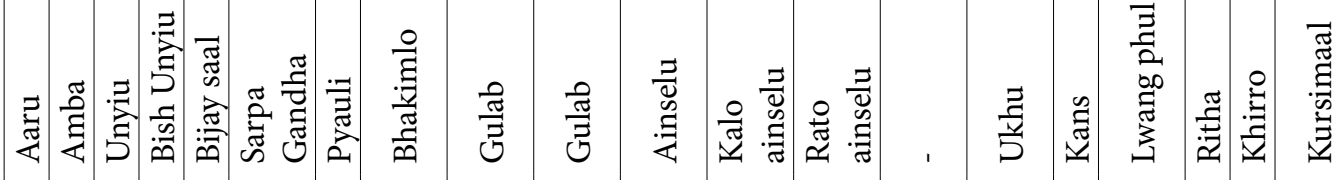

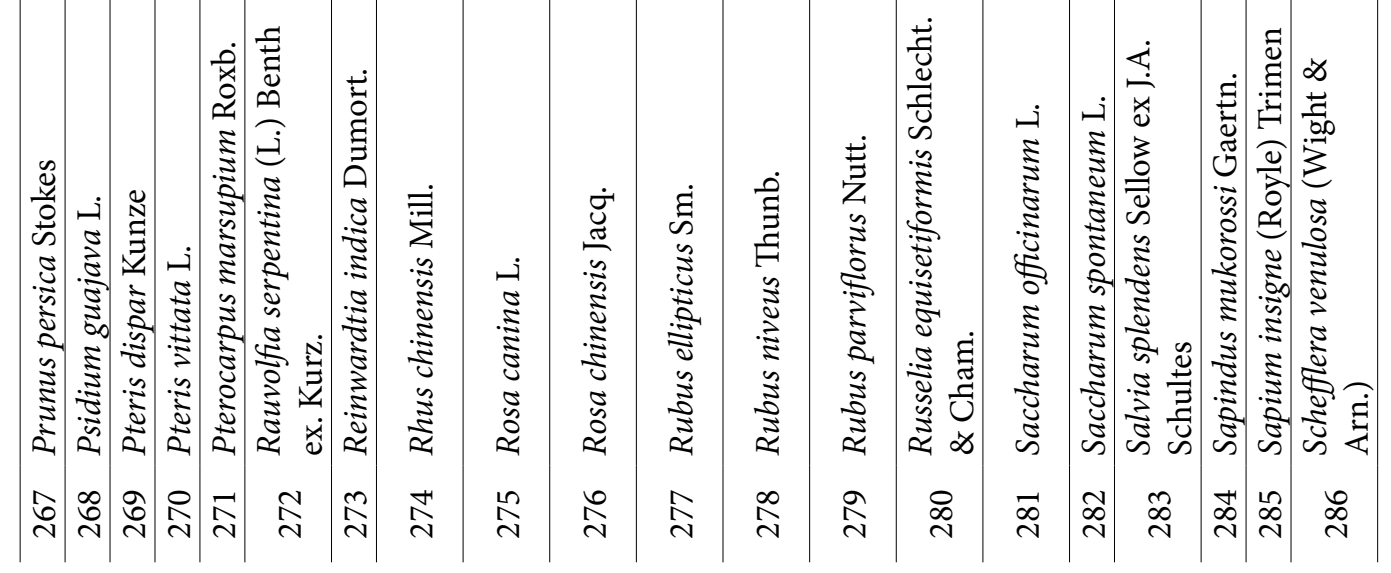




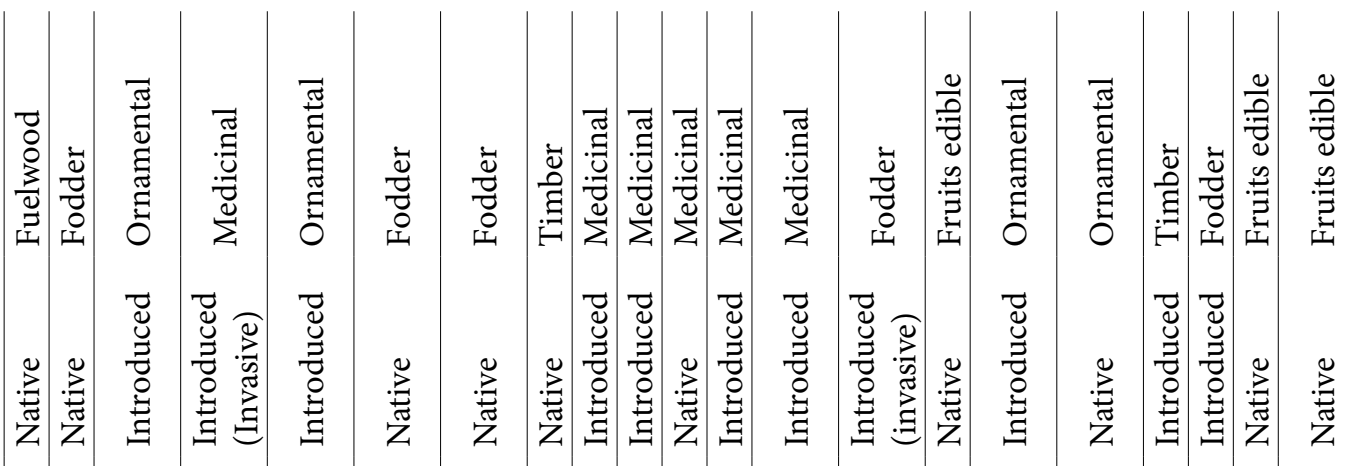

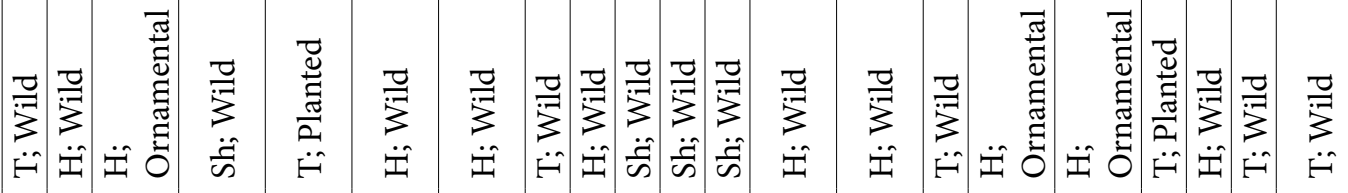

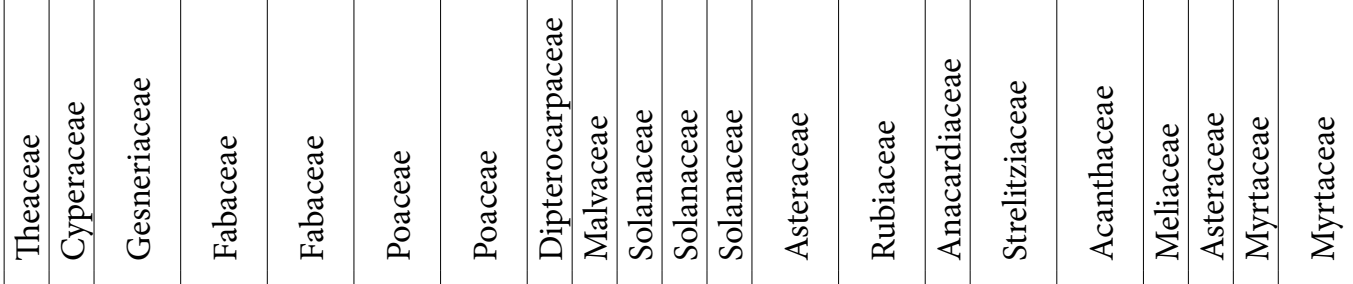

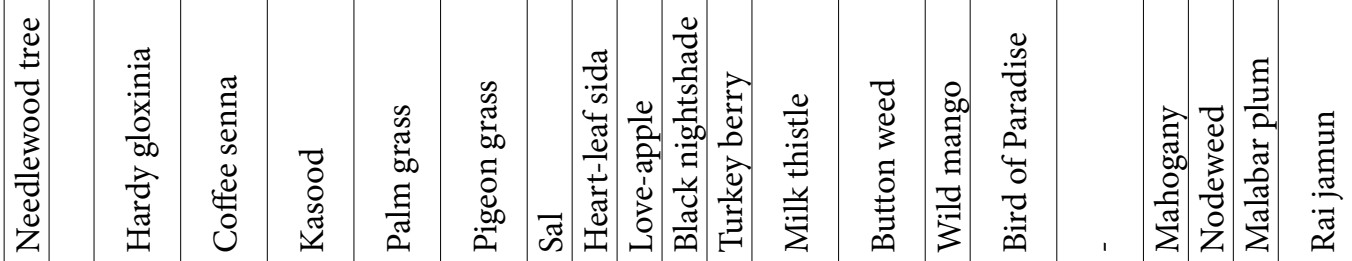

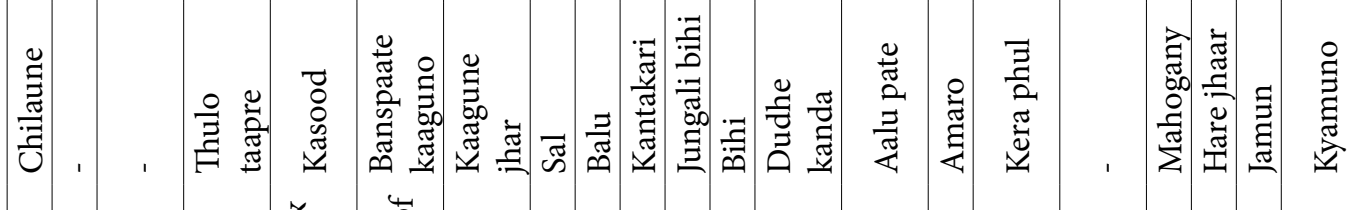

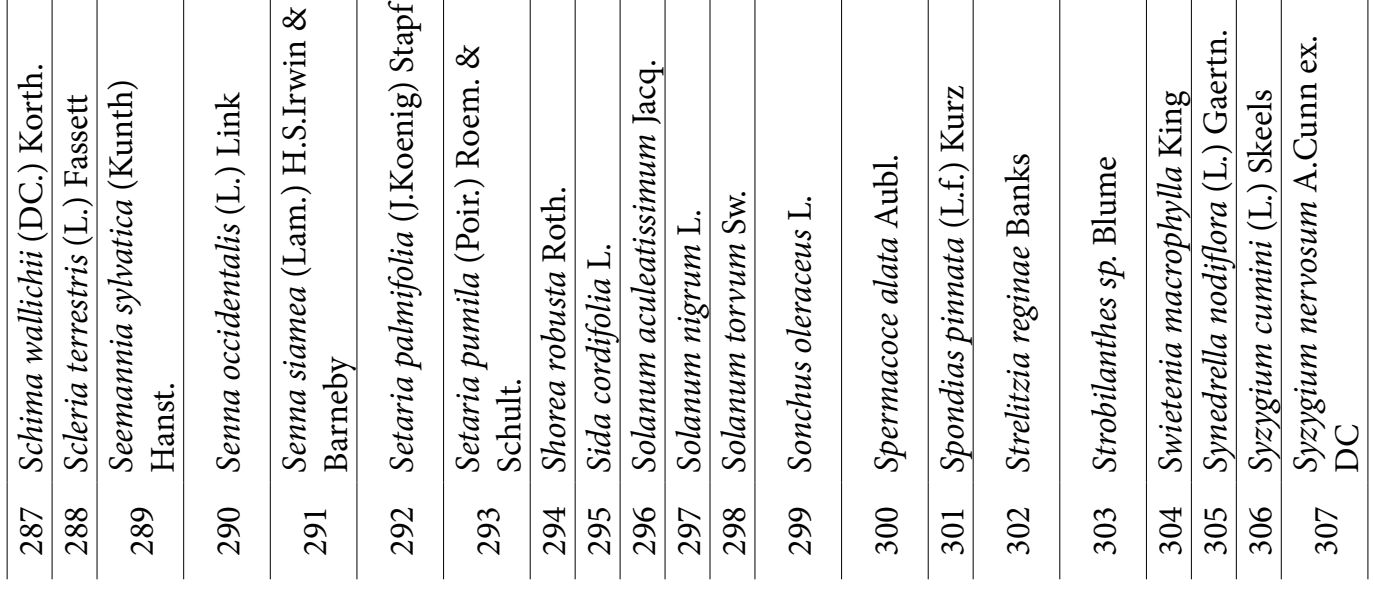




\begin{tabular}{|c|c|c|c|c|c|c|c|c|c|c|c|c|c|c|c|c|c|c|}
\hline 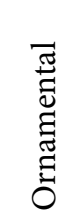 & 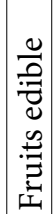 & 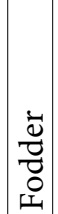 & 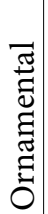 & 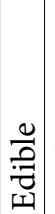 & $\begin{array}{l}\dot{\bar{\Xi}} \\
\text { ఏ } \\
\dot{\mathrm{H}}\end{array}$ & 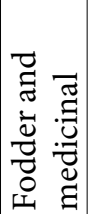 & 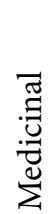 & 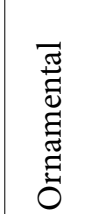 & 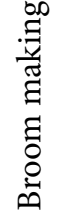 & 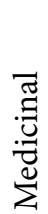 & 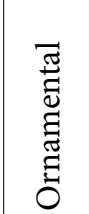 & 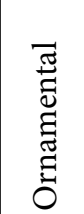 & $\mid \begin{array}{l}\bar{z} \\
\bar{z} \\
\overline{0} \\
0 \\
\dot{I}\end{array}$ & 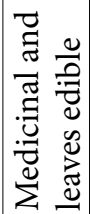 & 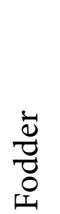 & $\frac{\ddot{\theta}}{\stackrel{\theta}{ت}}$ & 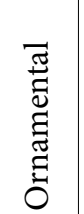 & 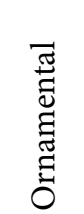 \\
\hline 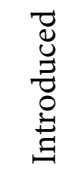 & 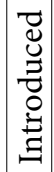 & $\begin{array}{l}\stackrel{\Xi}{\tilde{\Xi}} \\
\tilde{Z}\end{array}$ & 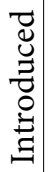 & 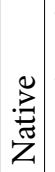 & 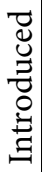 & $\underset{⿱ 亠 凶}{\stackrel{\Xi}{Z}}$ & 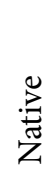 & $\begin{array}{l}\widetilde{\Xi} \\
\stackrel{U}{\Xi} \\
0 \\
\stackrel{\Xi}{\Xi}\end{array}$ & $\stackrel{\stackrel{D}{Z}}{\stackrel{\Xi}{Z}}$ & 売 & 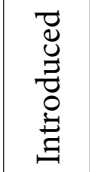 & 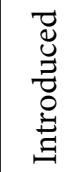 & 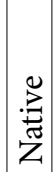 & 壳 & 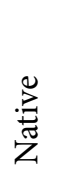 & 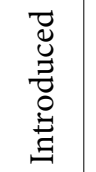 & 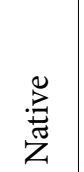 & 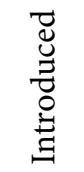 \\
\hline 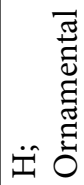 & 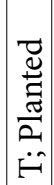 & 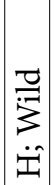 & 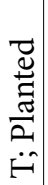 & $\begin{array}{c}\frac{0}{7} \\
3 \\
\dot{3} \\
\dot{I}\end{array}$ & 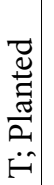 & 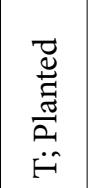 & 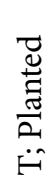 & 总 & 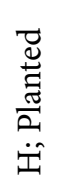 & $\begin{array}{l}\vec{\sigma} \\
\dot{3} \\
\dot{\vec{j}}\end{array}$ & 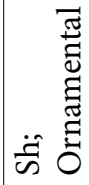 & 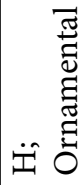 & $\begin{array}{l}\frac{0}{3} \\
\dot{3} \\
\dot{3}\end{array}$ & 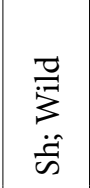 & $\begin{array}{l}\stackrel{0}{3} \\
\dot{3} \\
\ddot{j}\end{array}$ & 壴 & 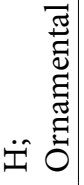 & 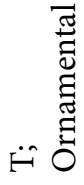 \\
\hline
\end{tabular}

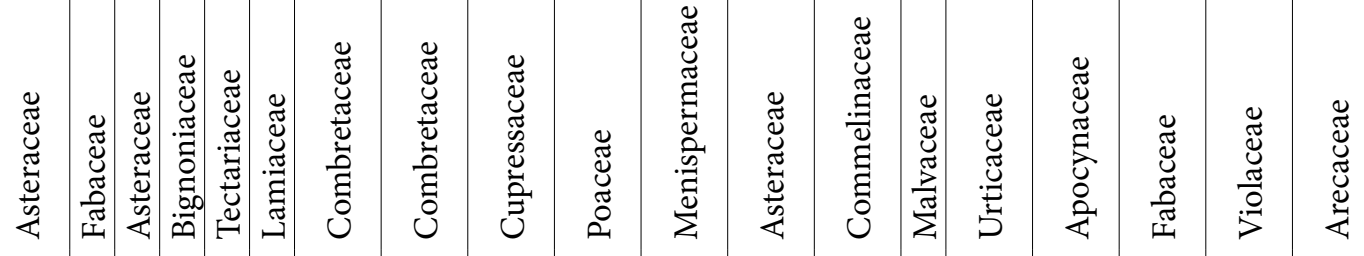

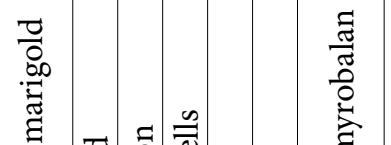

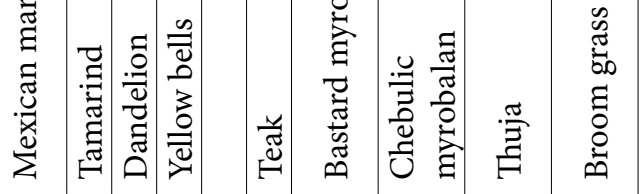

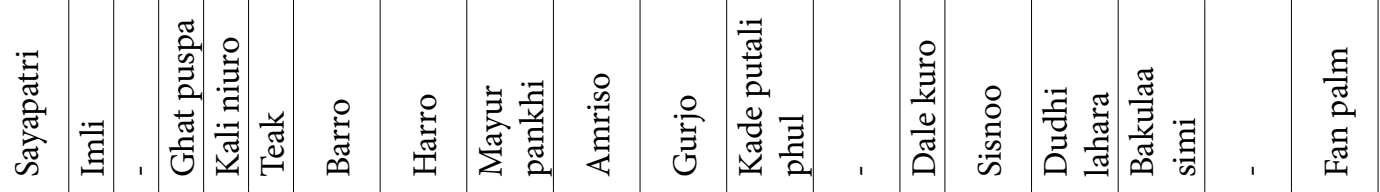

$$
\begin{aligned}
& \text {. }
\end{aligned}
$$

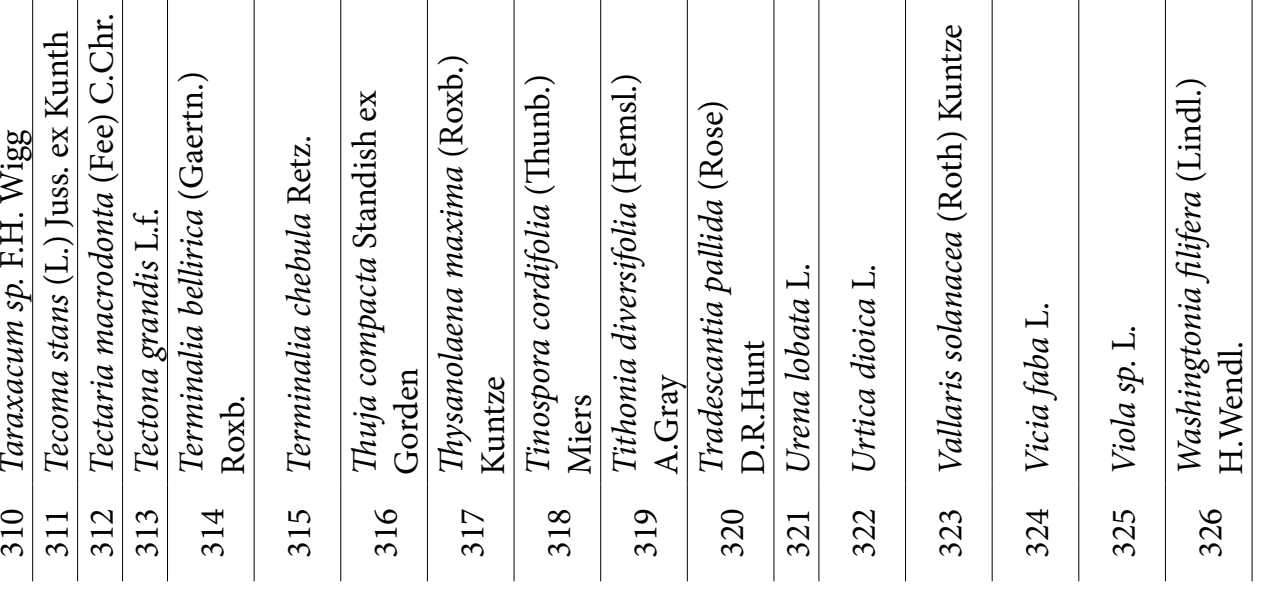




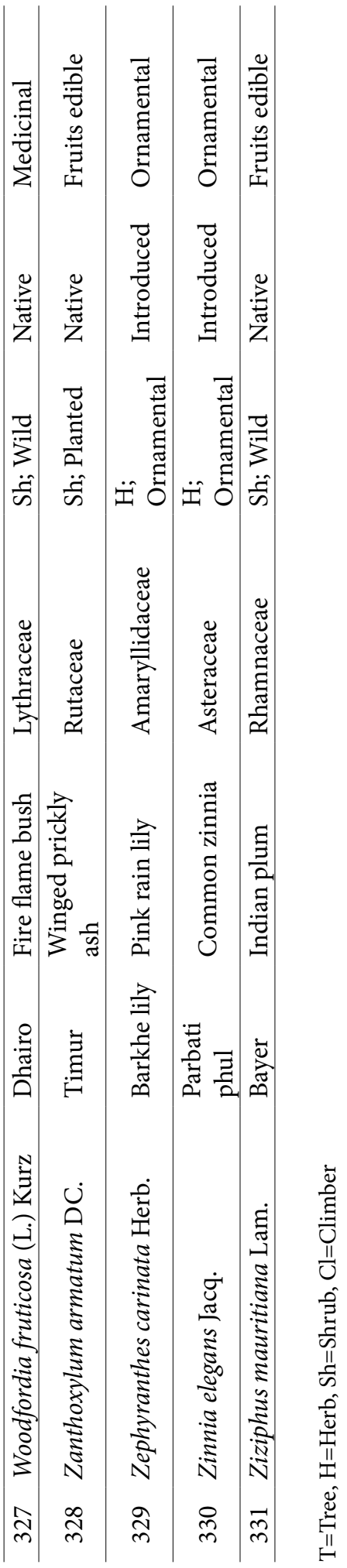




\section{Photo plates of flora}

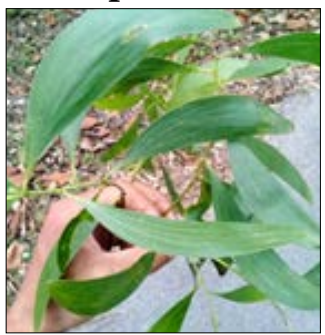

1. Acacia auriculiformis

(C)MS Miya

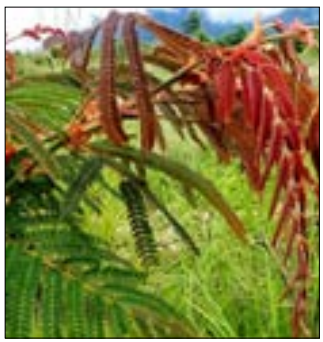

5. Albizia chinensis

(C)MS Miya

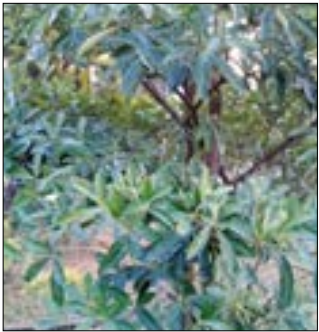

9. Alstonia scholaris

(C)MS Miya

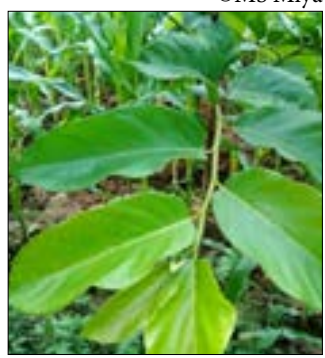

13. Artocarpus lakoocha

(C)MS Miya

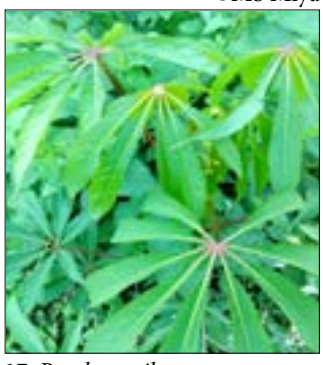

17. Bombax ceiba

(C)MS Miya

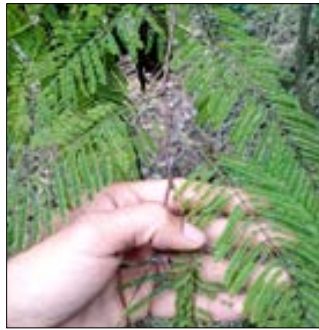

2.Acacia catechu

(C)MS Miya

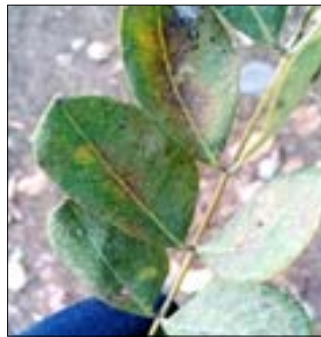

6. Albizia lebbeck

(C)MS Miya

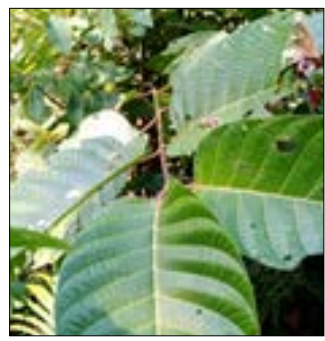

10. Anthocephalus cadamba

(C)MS Miya

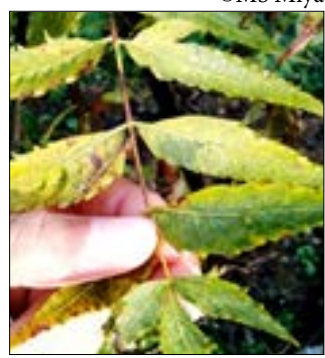

14. Azadirachta indica

(C)MS Miya

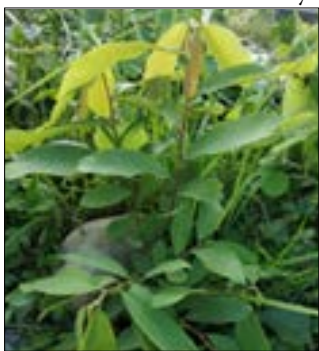

18. Bridelia retusa

(C) Gautam

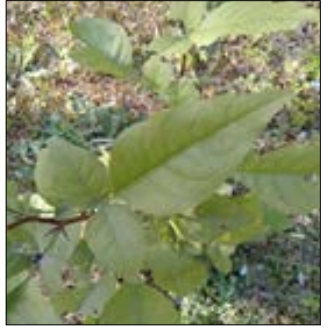

3. Aegle marmelos

(C)MS Miya

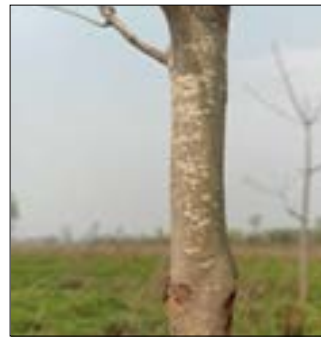

7. Albizia procera

CD Gautam

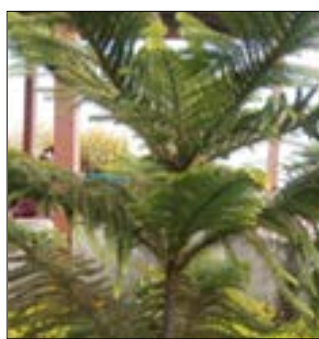

11. Araucaria columnaris

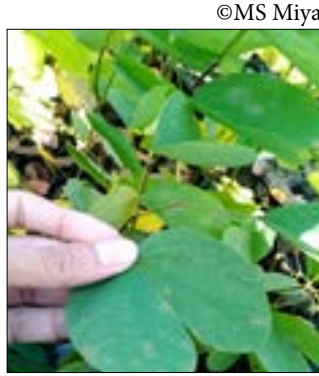

15. Bauhinia purpurea

(C)MS Miya

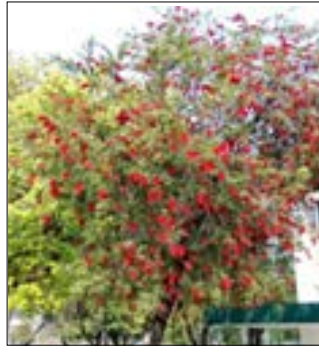

19. Callistemon citrinus

(C)MS Miya

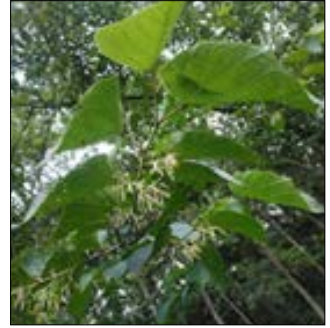

4. Alangium chinense

(C) Gautam

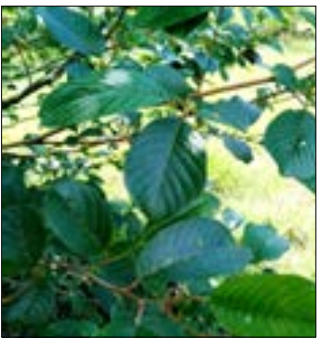

8. Alnus nepalensis

(C)MS Miya

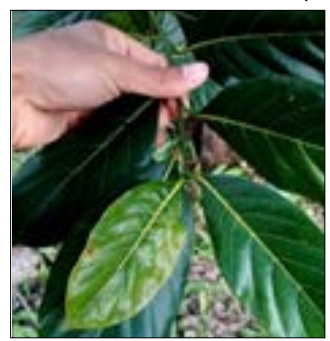

12. Artocarpus heterophyllus

(C)MS Miya

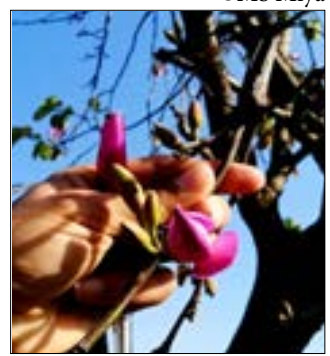

16. Bauhinia variegata

(C)MS Miya

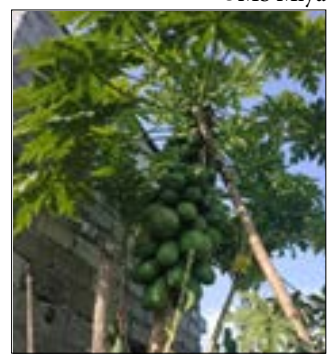

20. Carica papaya

(C) Gautam 


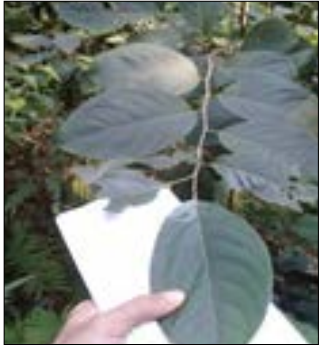

21. Casearia graveolens

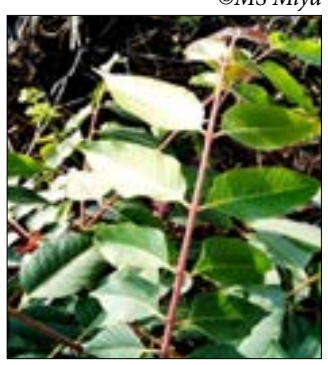

25. Cedrela toona

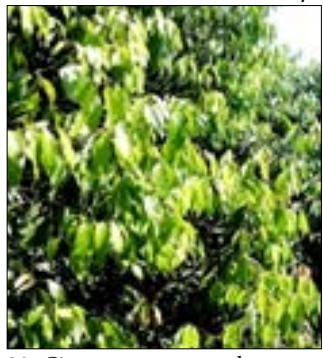

29. Cinnamomum tamala

(c) MS Miya

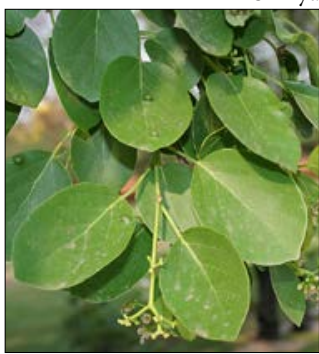

33. Cordia dichotoma

(c) J.M. Garg

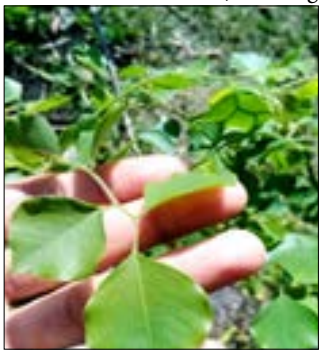

37. Dalbergia sissoo

(C)MS Miya

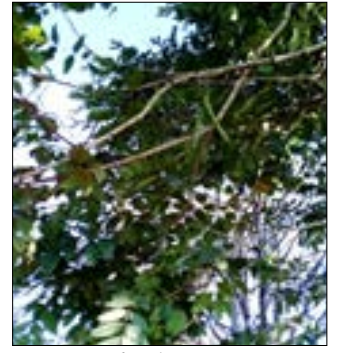

22. Cassia fistula

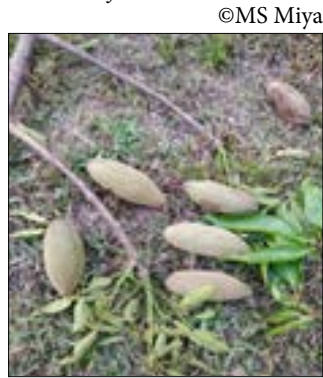

26. Ceiba pentandra

(C) Gautam

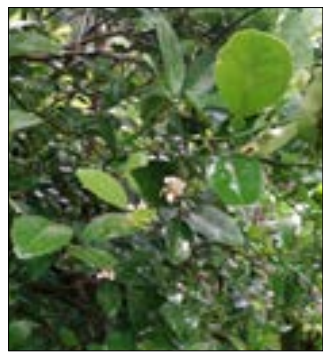

30. Citrus limon

(CD Gautam

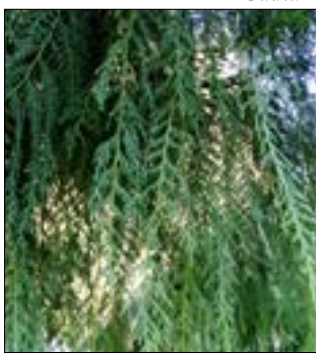

34. Cupressus torulosa

() MS Miya

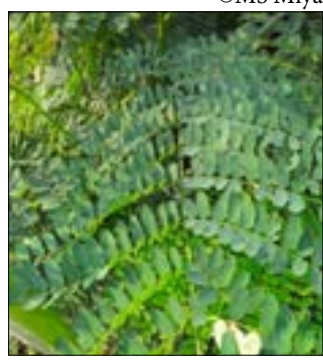

38. Delonix regia

(C) Gautam

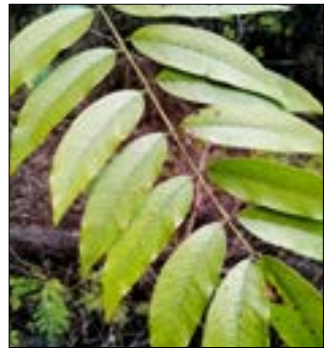

23. Cassia javanica

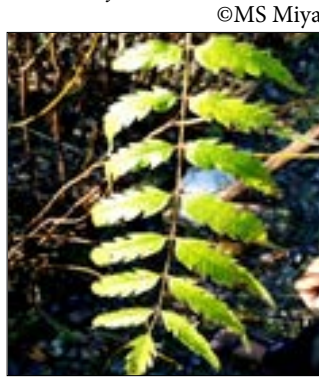

27. Choerospondias axillaris

(c)MS Miya

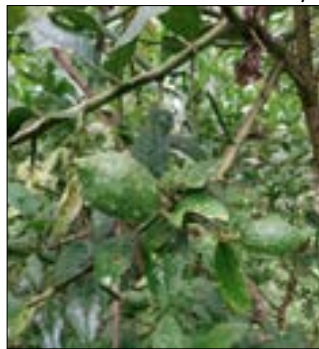

31. Citrus medica

(C) Gautam

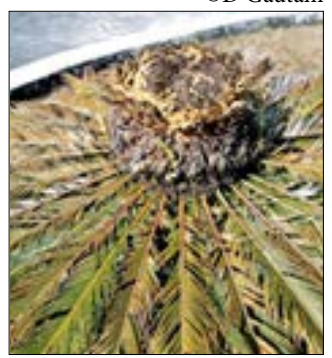

35. Cycas revoluta

(c)MS Miya

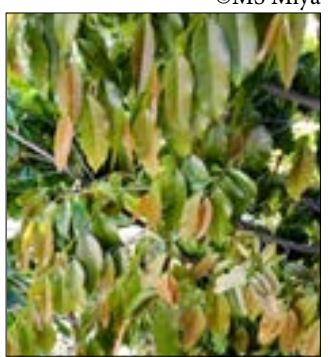

39. Diospyros Montana

()MS Miya

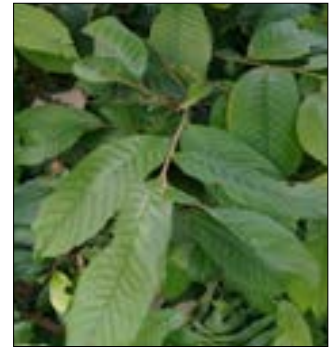

24. Castanopsis indica

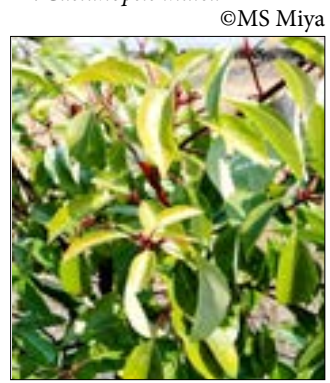

28. Cinnamomum camphora

()MS Miya

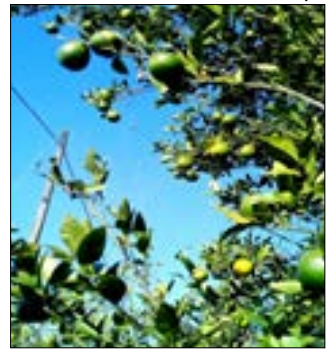

32. Citrus reticulate

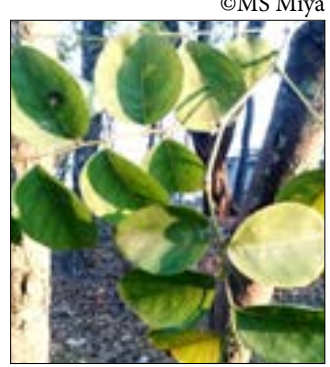

36. Dalbergia latifolia

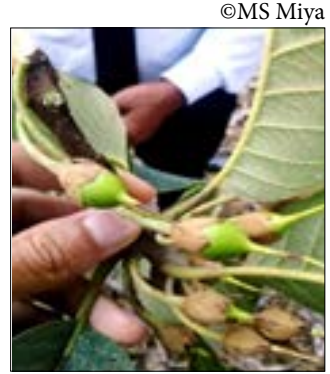

40. Diploknema butyracea

(c)MS Miya 


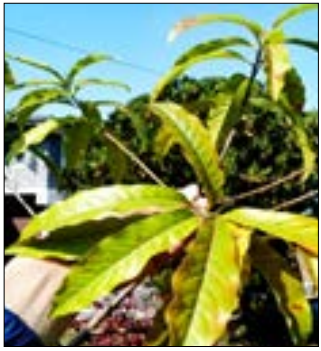

41. Elaeocarpus sphaericus

(c)MS Miya

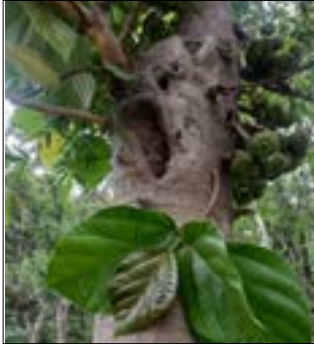

45. Ficus auriculata

(CD Gautam

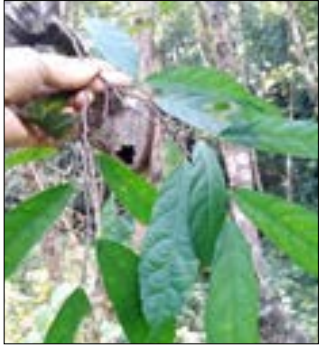

49. Ficus clavata

(C)MS Miya

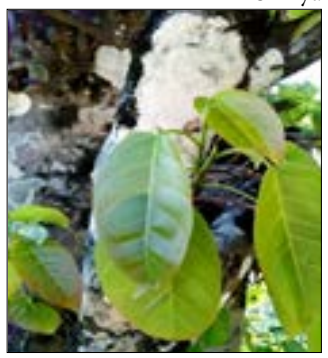

53. Ficus lacor

(c)MS Miya

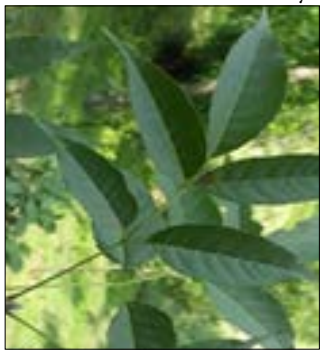

57. Fraxinus floribunda

(c) G Gautam

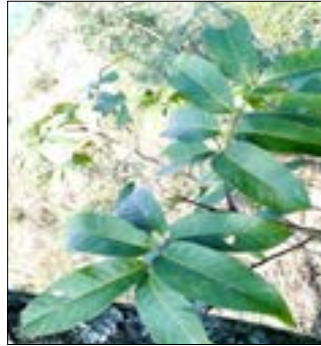

42. Engelhardtia spicata

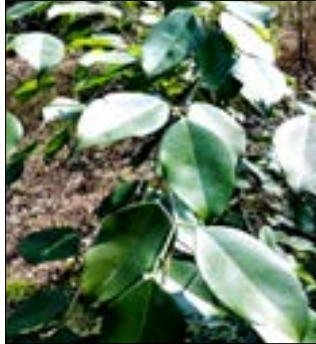

46. Ficus benjamina

(c)MS Miya

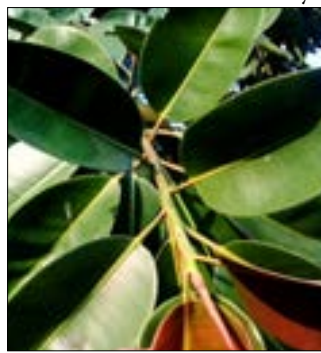

50. Ficus elastica

(c)MS Miya

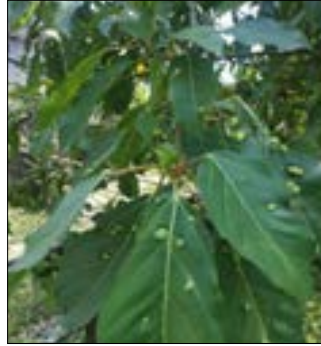

54. Ficus racemosa

(c)D Gautam

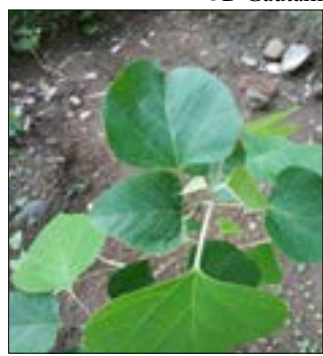

58. Gmelina arborea

(c) Gautam

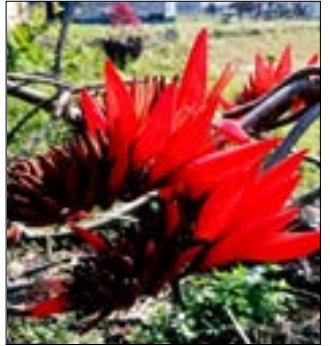

43. Erythrina stricta

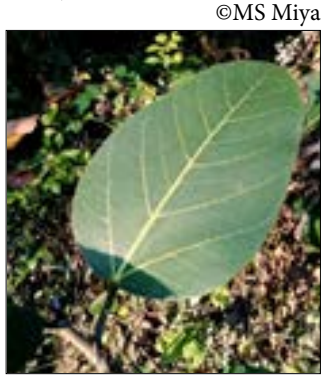

47. Ficus benghalensis

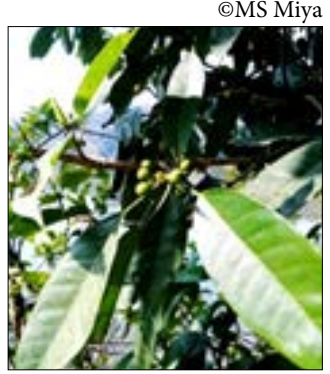

51. Ficus glaberrima

(c)MS Miya

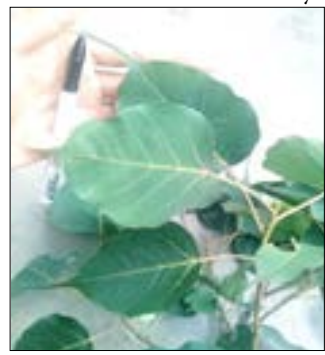

55. Ficus religios

(C)MS Miya

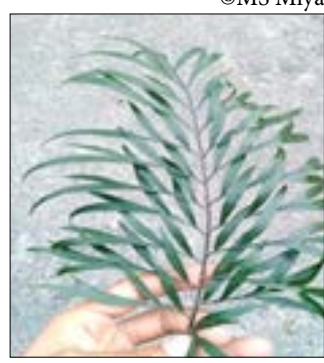

59. Grevillea robusta

()MS Miya

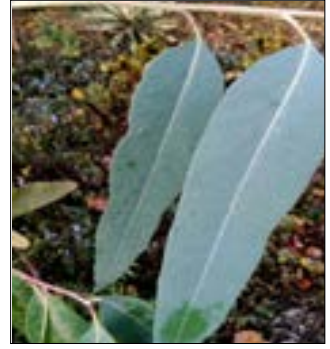

44. Eucalyptus camaldulensis

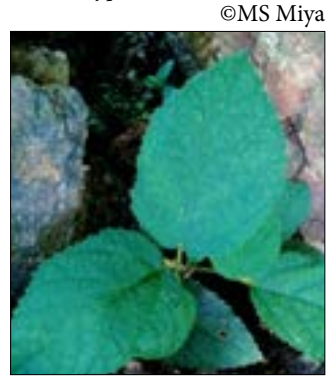

48. Ficus carica

(C)MS Miya

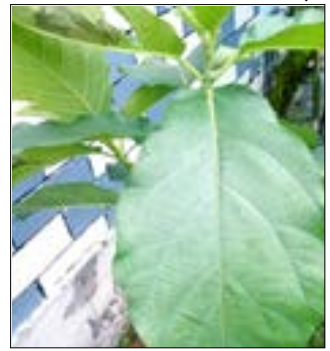

52. Ficus hispida

(c)MS Miya

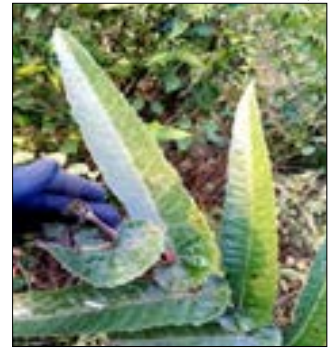

56. Ficus semicordata

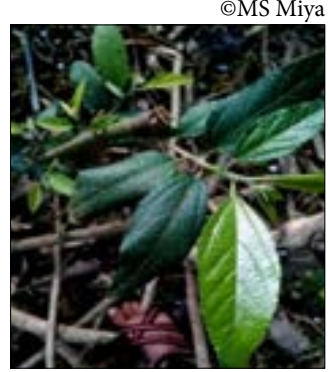

60. Grewia sp.

()MS Miya 


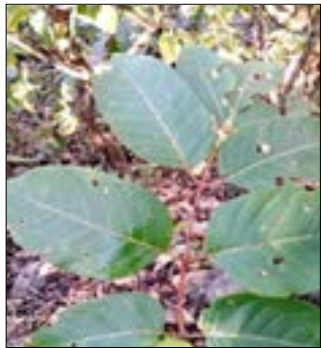

61. Heynea trijuga

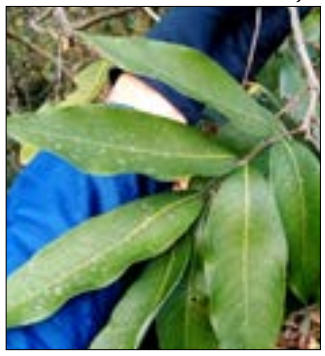

65. Litchi chinensis $\quad$ (MS Miya

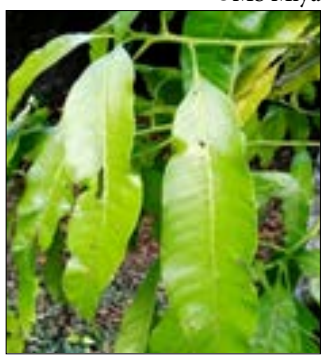

69. Mangifera indica

(c)MS Miya

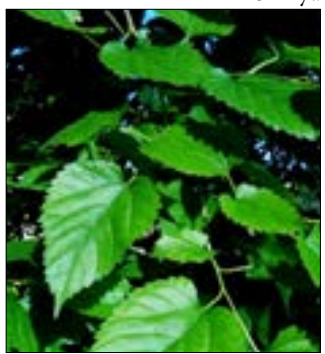

73. Morus alba

(C)MS Miya

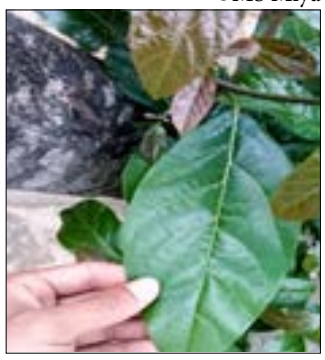

77. Persea Americana

(c)MS Miya

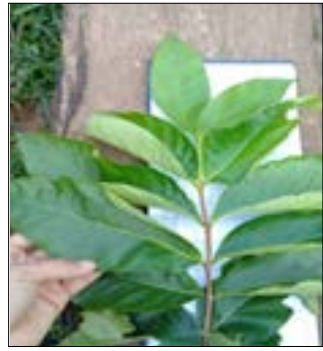

62. Holarrhena pubescens

(c)MS Miya

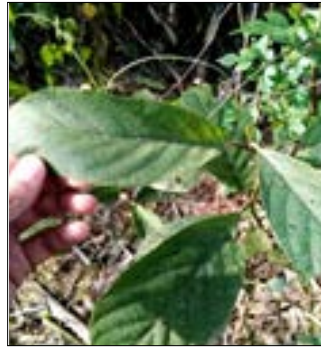

66. Litsea monopetala

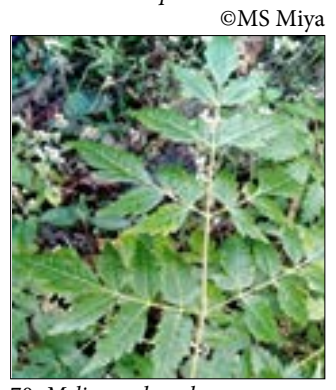

70. Melia azedarach

(c)MS Miya

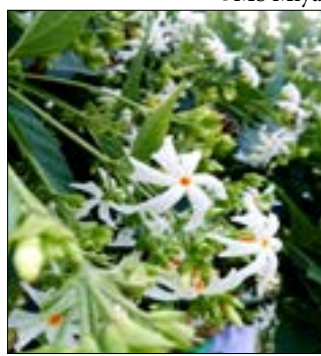

74. Nyctanthes arbor-tristis

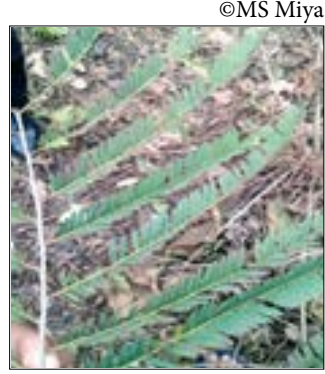

78. Phyllanthus emblica

()MS Miya

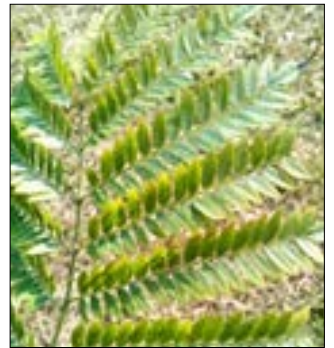

63. Jacaranda mimosifolia

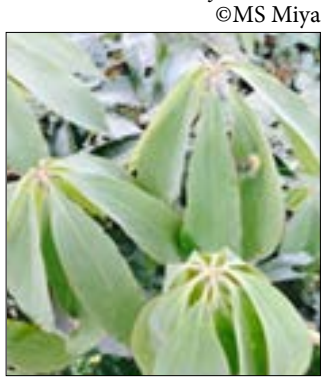

67. Machilus $s p$.

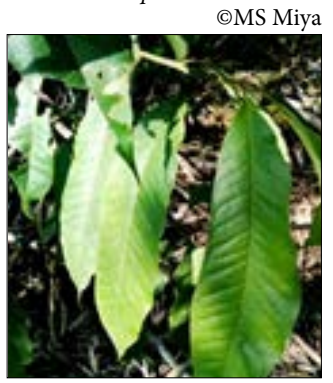

71. Michelia champaca

( )MS Miya

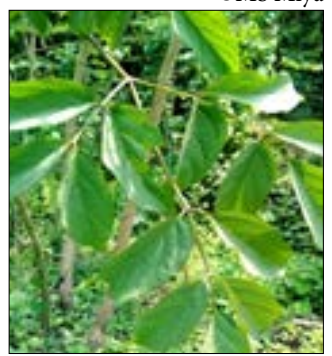

75. Oroxylum indicum

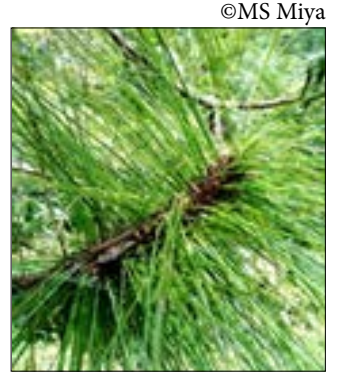

79. Pinus roxburghii

(c)MS Miya

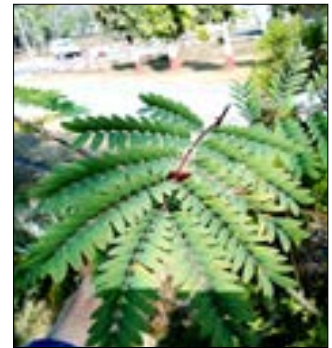

64. Leucaena leucocephala

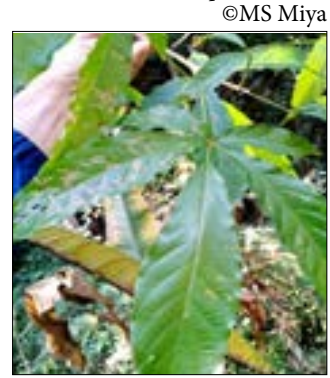

68. Macropanax undulates

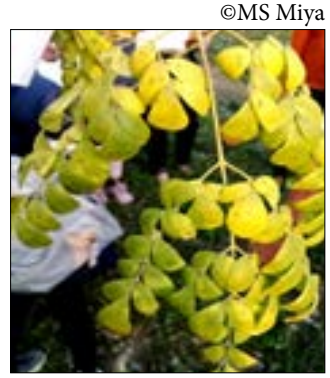

72. Moringa oleifera

(c)MS Miya

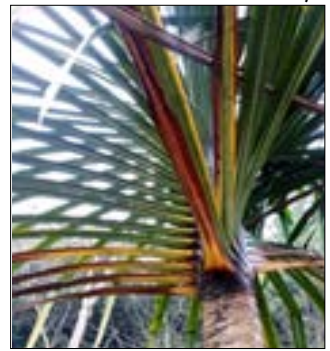

76. Pandanus nepalensis

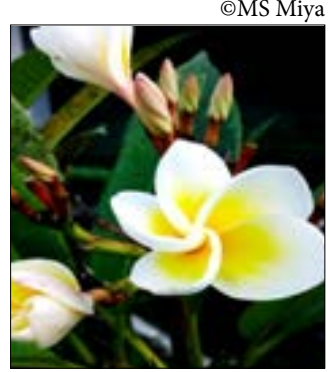

80. Plumeria alba

()MS Miya 


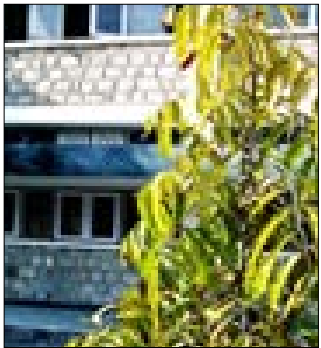

81. Polyalthia longifolia

(C) MS Miya

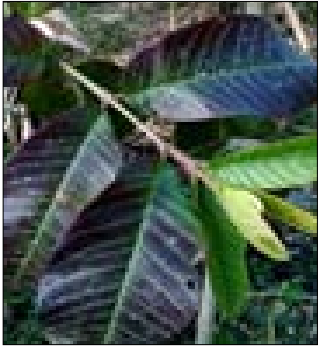

85. Psidium guajava

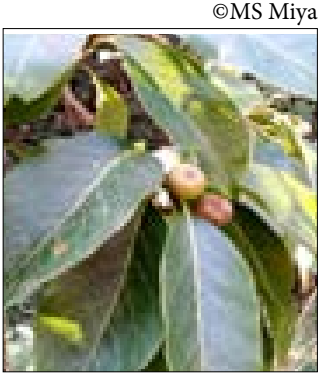

89. Schima wallichi

(C)MS Miya

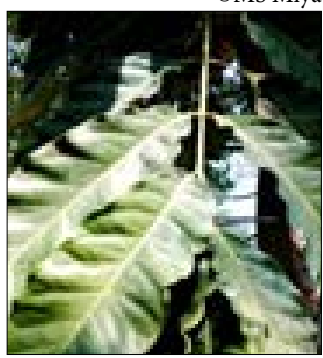

93. Swietenia macrophylla

(C)MS Miya

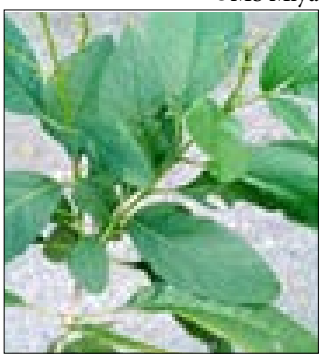

97. Tecoma stans

(C)MS Miya

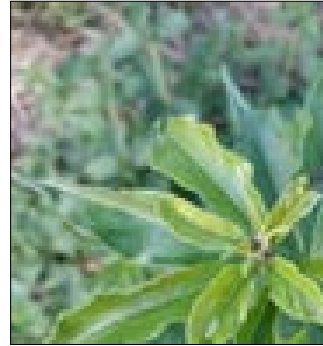

82. Premna barbata

(C) Gautam

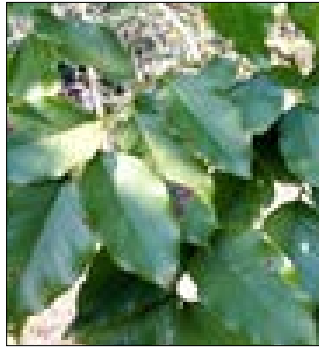

86. Pterocarpus marsupium

(C)MS Miya

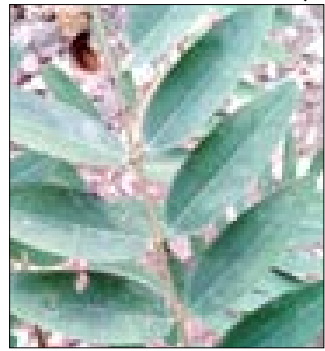

90. Senna siamea

(C)MS Miya

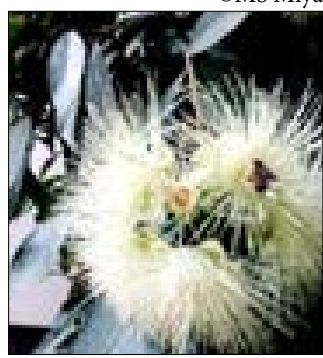

94. Syzygium cumin

(C)MS Miya

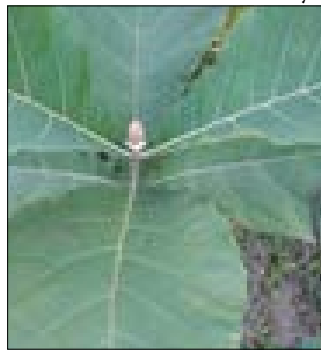

98. Tectona grandis

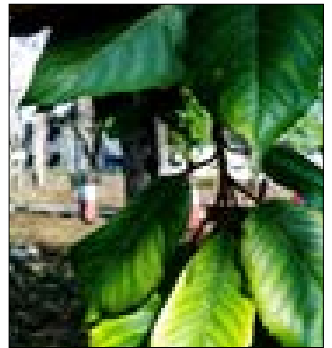

83. Prunus cerasoides

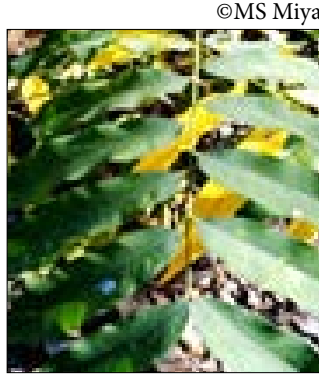

87. Sapindus mukorossi

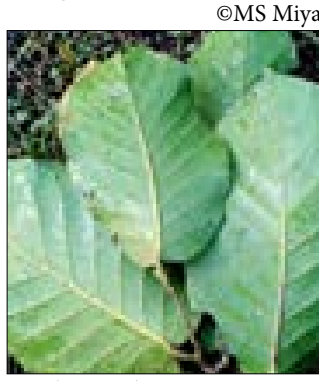

91. Shorea robusta

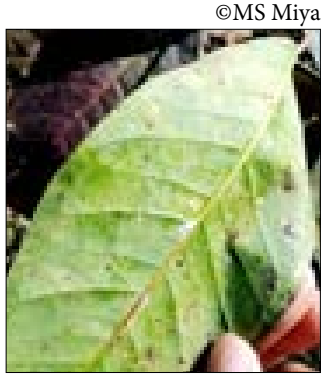

95. Syzygium nervosum

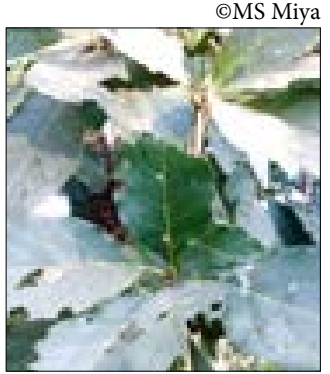

99. Terminalia belerica

(C)MS Miya

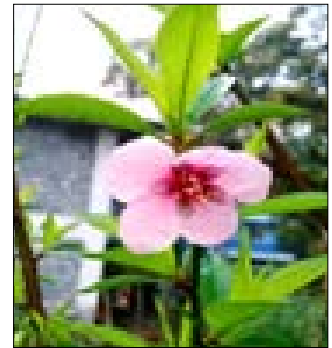

84. Prunus persica $\quad$ (C)MS Miya

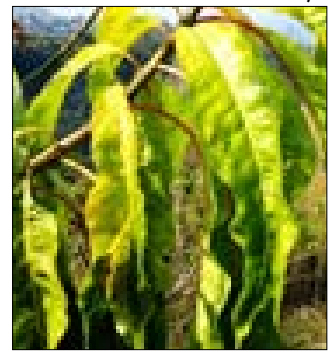

88. Sapium insigne

(C)MS Miya

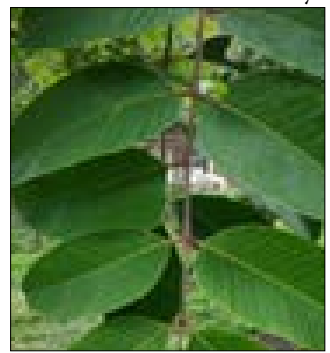

92. Spondias pinnata

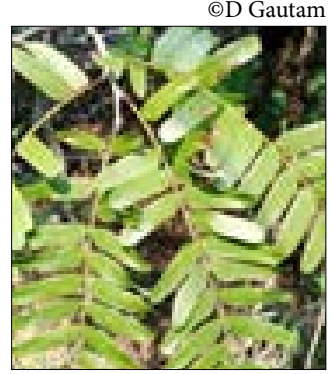

96. Tamarindus indica

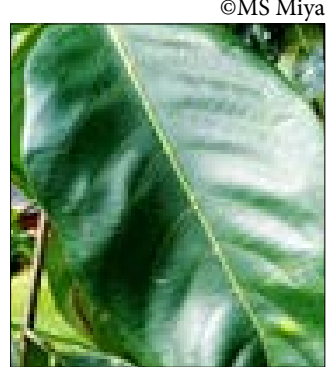

100. Terminalia chebula

(c) MS Miya 


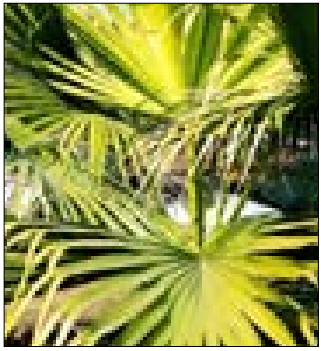

101. Washingtonia filifera

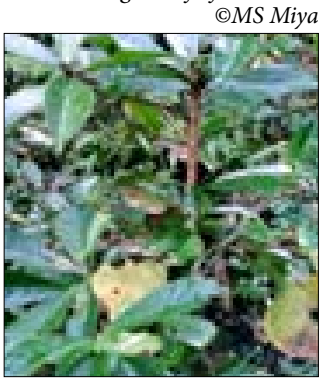

4. Ardisia macrophylla

(C)MS Miya

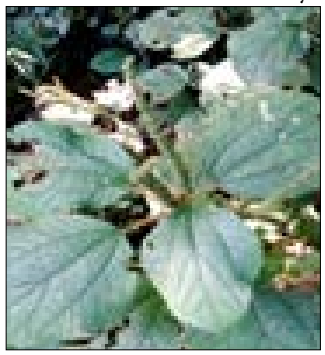

8. Boehmeria platyphylla

(C)MS Miya

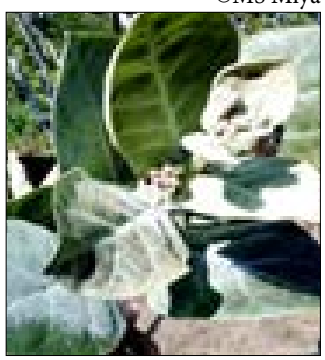

12. Calotropis gigantean

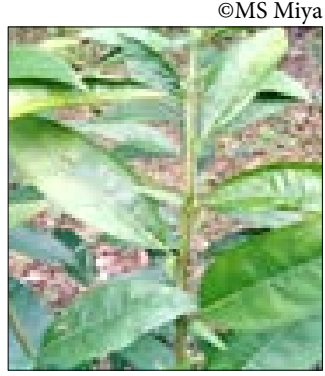

16. Cestrum nocturnum

(c)MS Miya

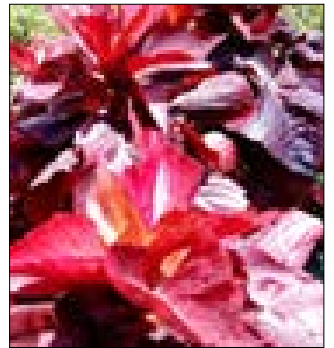

1. Acalypha wilkesiana

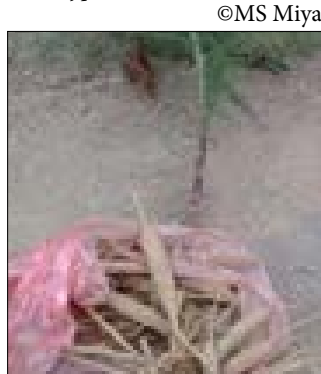

5. Asparagus racemosus

() MS Miya

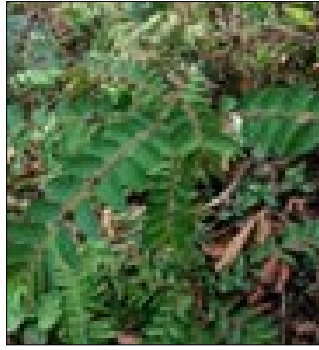

9. Breynia sp.

(c)MS Miya

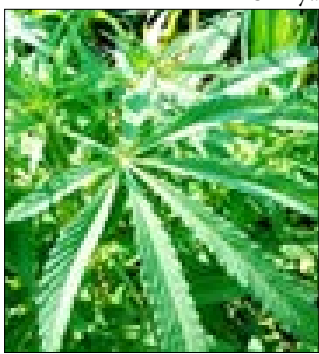

13. Cannabis sativa

๑)MS Miya

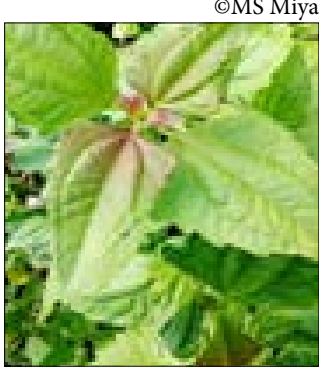

17. Chromolaena odorata

(c)MS Miya

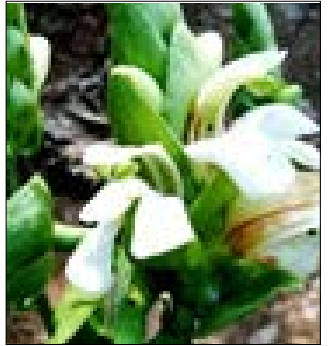

2. Adhatoda vasica

(c)MS Miya

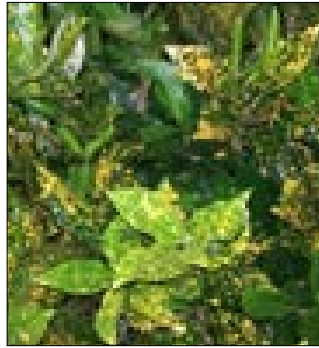

6. Aucuba japonica

(c) Gautam

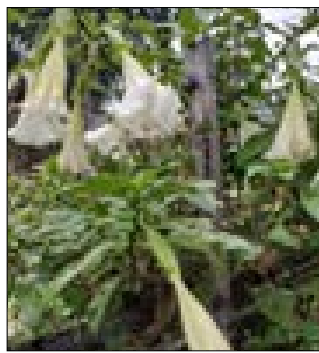

10. Brugmansia

(c) G Gautam

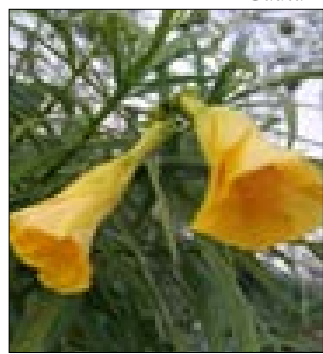

14. Cascabela thevetia

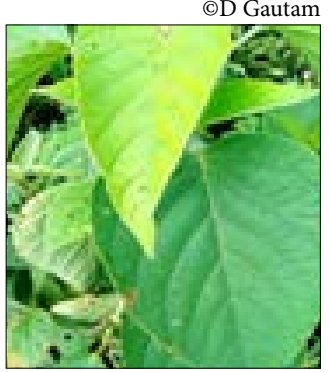

18. Clerodendrum infortunatum

cMS Miya

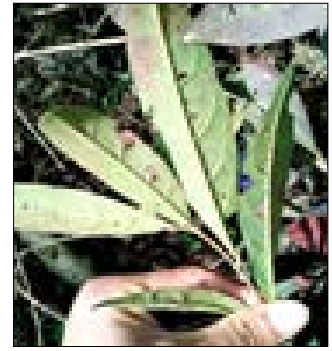

3. Antidesma acidum

() MS Miya

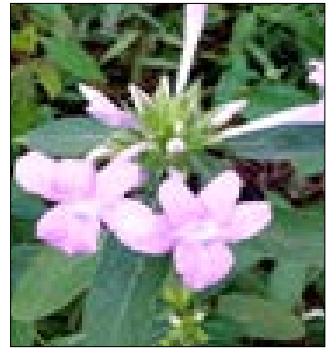

7. Barleria cristata

(C)MS Miya

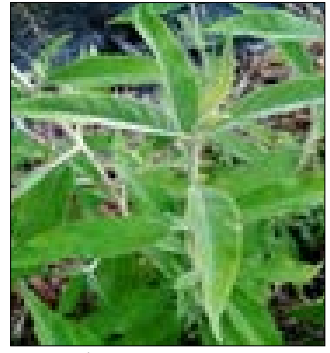

11. Budjeja asiatica

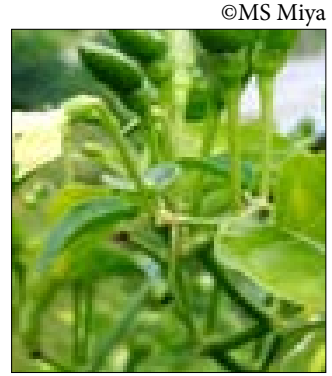

15. Capsicum sp.

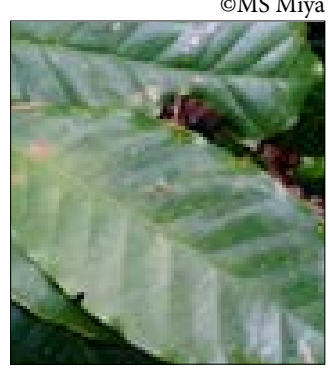

19. Coffea arabica

(C)MS Miya 


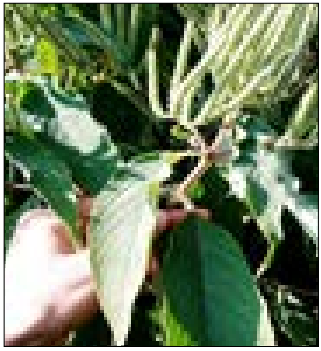

20. Colebrookea oppositifolia (C)MS Miya

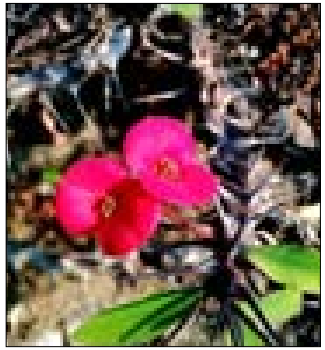

24. Euphorbia milii

(c)MS Miya

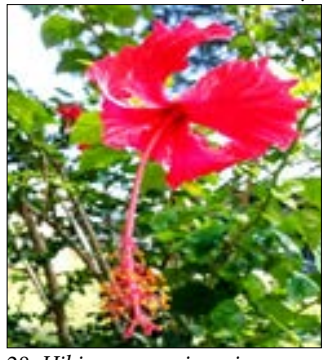

28. Hibiscus rosa-sinensis

(C)MS Miya

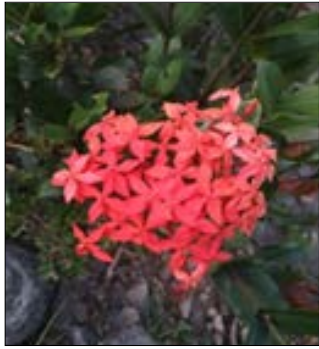

32. Ixora sp.

(c) Gautam

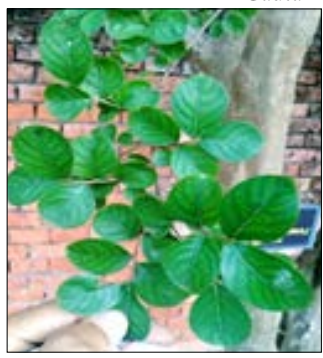

36. Lagerstroemia indica

(c)MS Miya

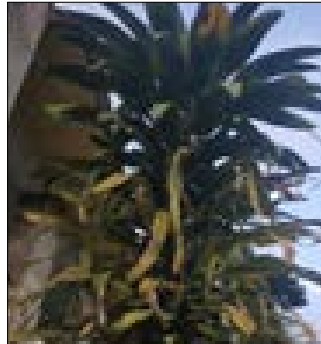

21. Cordyline fruticosa

(c) Gautam

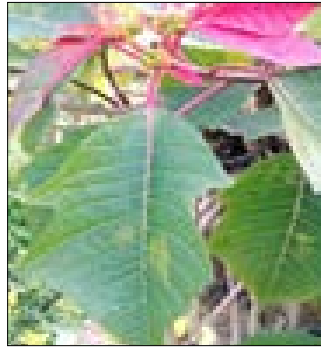

25. Euphorbia pulcherrima

(C)MS Miya

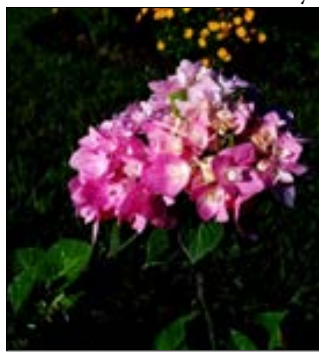

29. Hydrangea sp.

(C)MS Miya

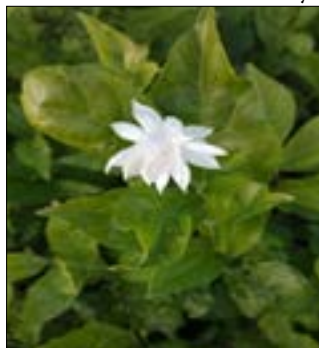

33. Jasminum sambac

(c) Gautam

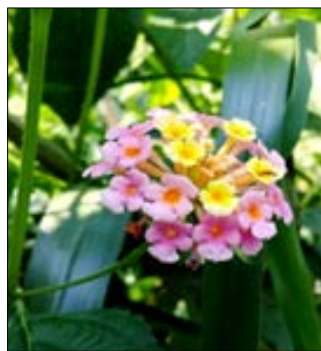

37. Lantana camara

(c)MS Miya

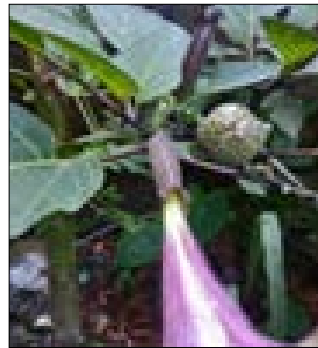

22. Datura metel

(c)D Gautam

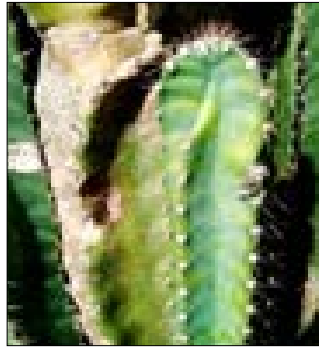

26. Euphorbia royleana

(C)MS Miya

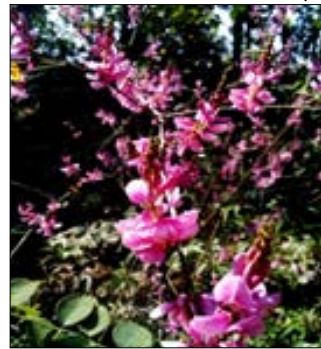

30. Indigofera $s p$.

๑MS Miya

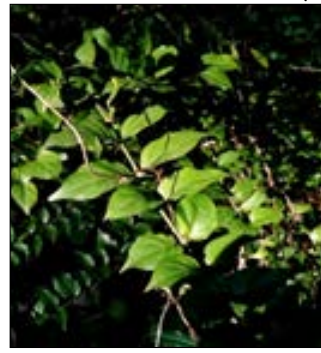

34. Jasminum sp.

(c)MS Miya

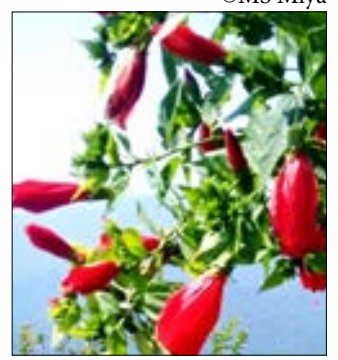

38. Malvaviscus arboreus

(c) MS Miya

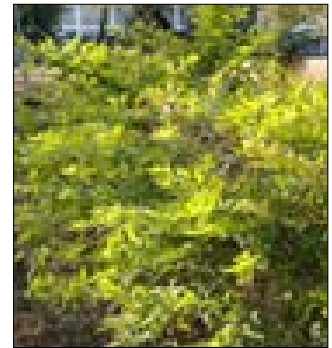

23. Duranta erecta

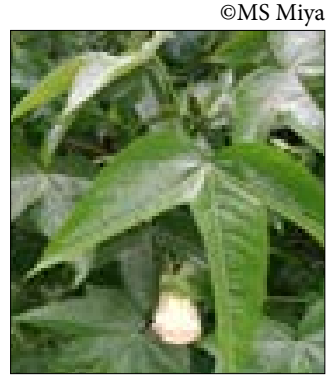

27. Gossypium arboreum

(CD Gautam

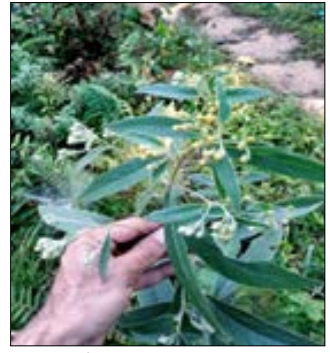

31. Inula cappa

(C)MS Miya

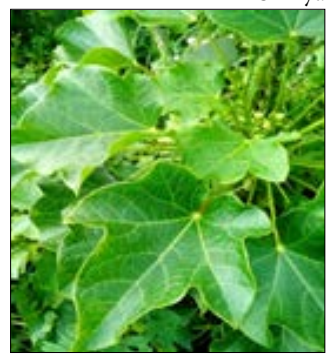

35. Jatropha curcas

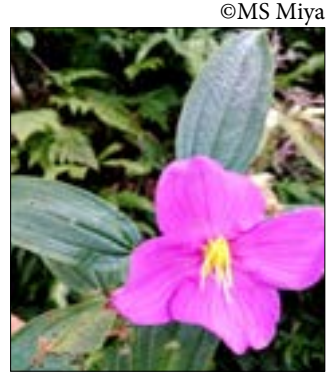

39. Melastoma malabathricum

(c)MS Miya 


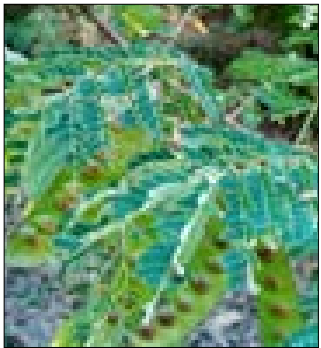

40. Mimosa rubicaulis

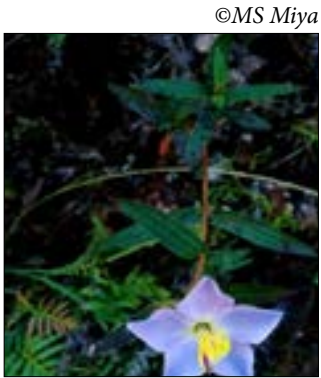

44. Osbeckia nepalensis

(C)MS Miya

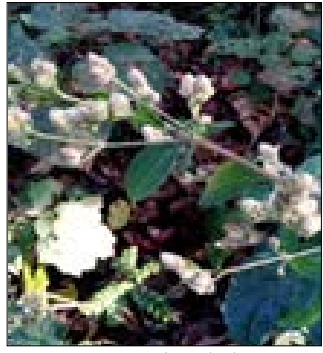

48. Pogostemon benghalensis

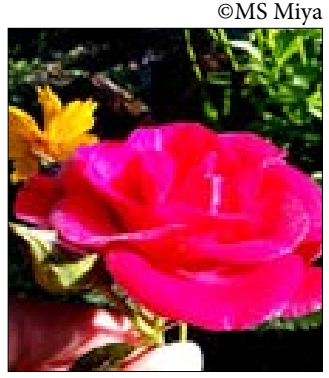

52. Rosa chinensis

(C)MS Miya

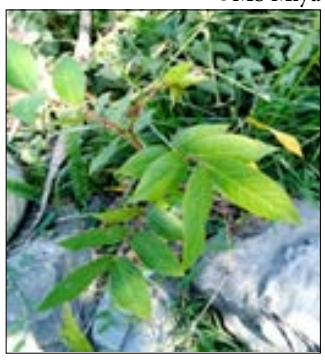

56. Senna occidentalis

(C)MS Miya

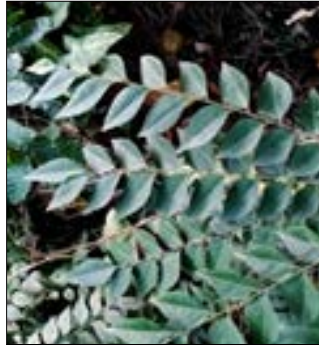

41. Murraya koenigi

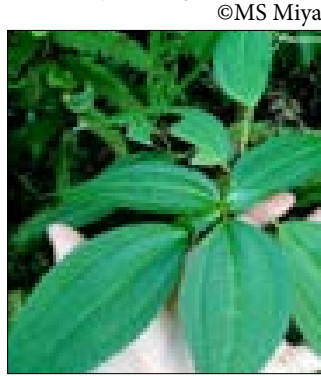

45. Pilea $s p$

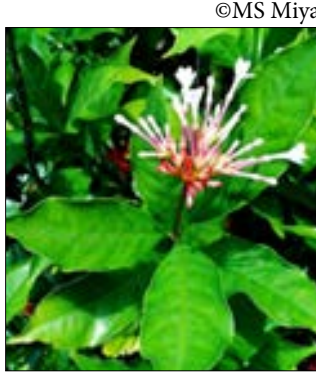

49. Rauvolfia serpentina

(C)MS Miya

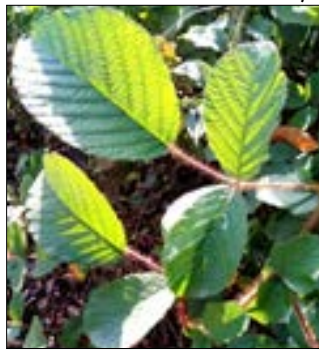

53. Rubus ellipticus

(C)MS Miya

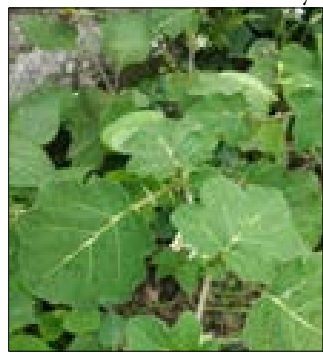

57. Solanum aculeatissimum

(C) Gautam

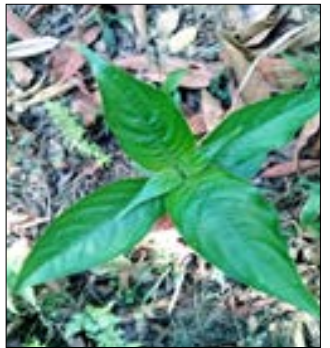

42. Mussaenda macrophylla

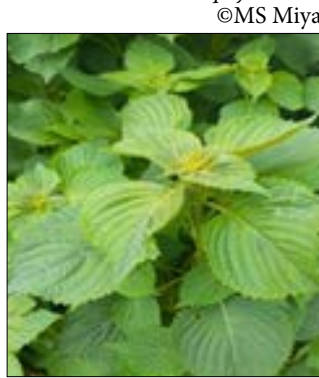

46. Perilla frutescens

CD Gautam

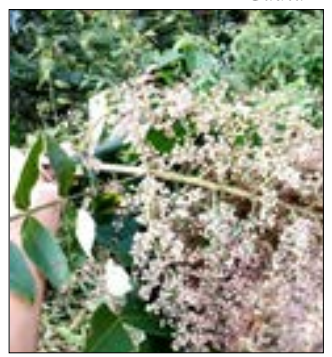

50. Rhus chinensis

(C)MS Miya

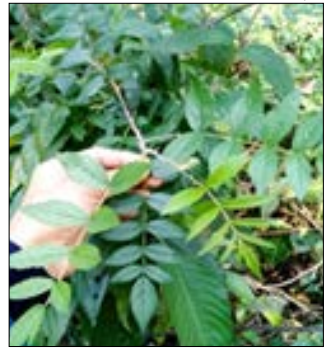

54. Rubus niveus

(C)MS Miya

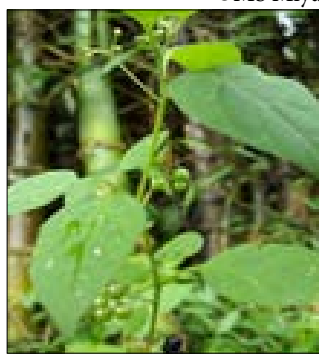

58. Solanum nigrum

(C)D Gautam

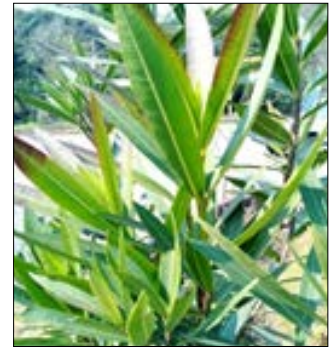

43. Nerium oleander

(C)MS Miya

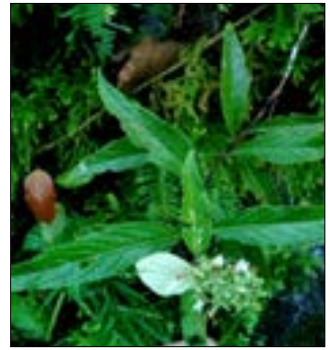

47. Plantostoma coloratum

(C)MS Miya

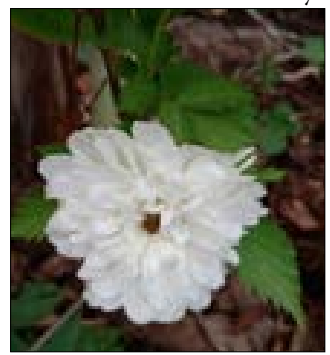

51. Rosa canina

(C) Gautam

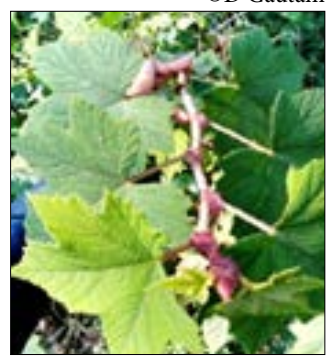

55. Rubus parviflorus

(C)MS Miya

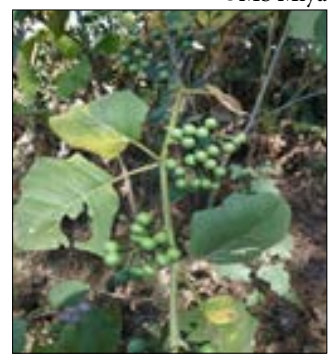

59. Solanum torvum

(C)D Gautam 


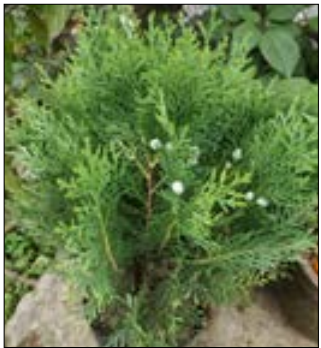

60. Thuja compacta

(C)D Gautam

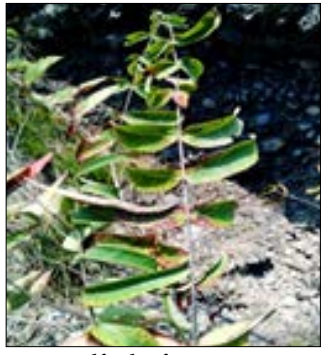

64. Woodfordia fruticosa

(C)MS Miya

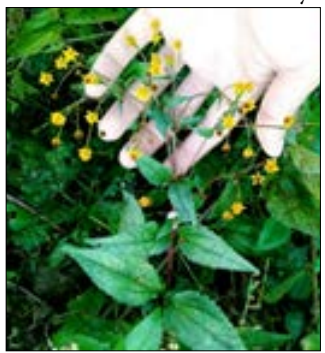

2. Acmella paniculata

(C)MS Miya

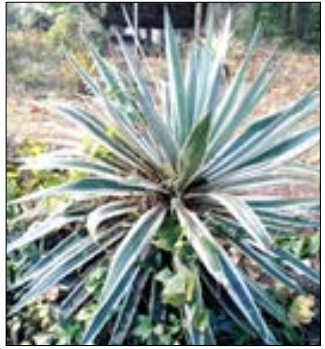

6. Agave American

(C)MS Miya

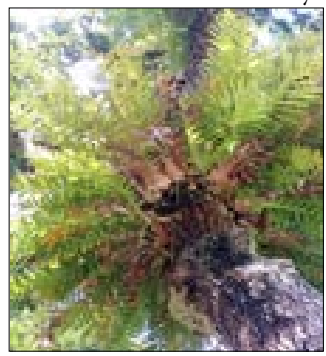

10. Aglaomorpha quercifolia

(C)MS Miya

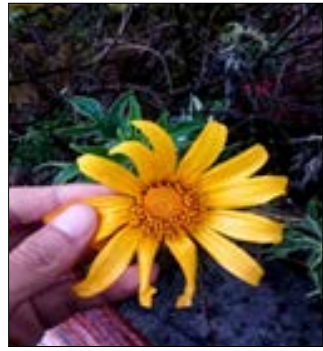

61. Tithonia diversifolia

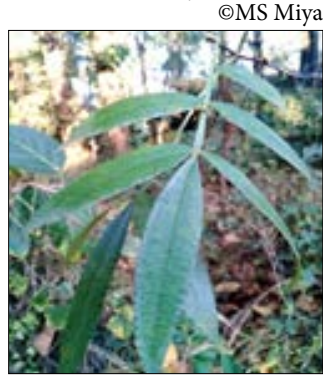

65. Zanthoxylum armatum

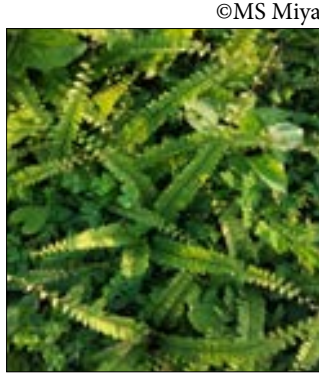

3. Adiantum caudatum

(C) Gautam

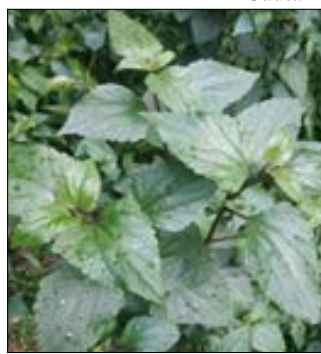

7. Ageratina adenophora

CD Gautam

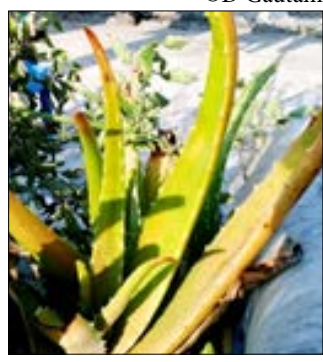

11. Aloe vera

(C)MS Miya

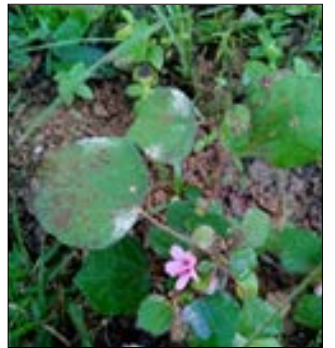

62. Urena lobata

(C)MS Miya

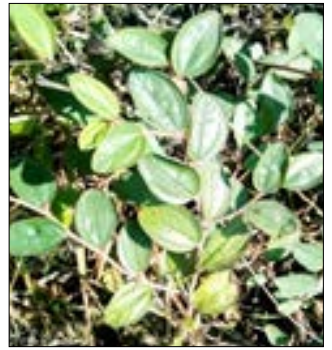

66. Ziziphus mauritiana

CMS Miya

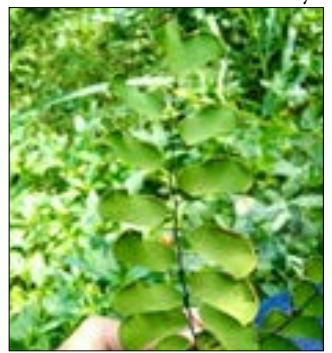

4. Adiantum philippense

(C)MS Miya

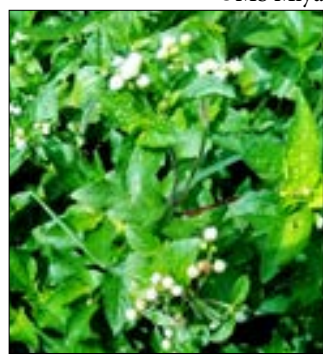

8. Ageratum conyzoides

(c)MS Miya

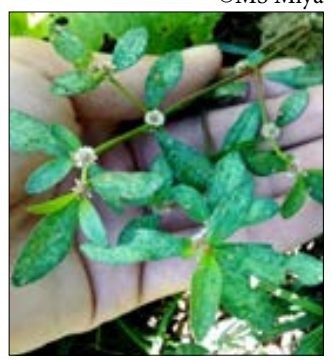

12. Alternatharia philoxeroides

(c)MS Miya

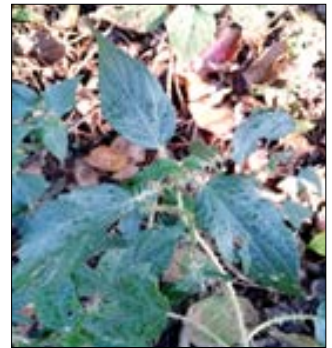

63. Urtica dioica

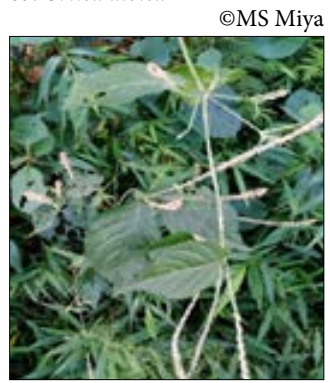

1. Achyranthes aspera

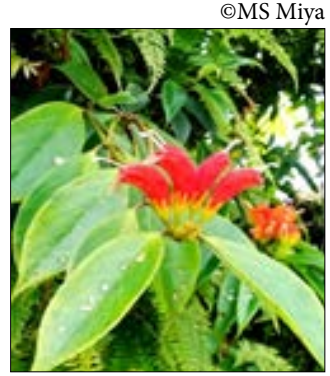

5. Aeschynanthus parviflorus

(C)MS Miya

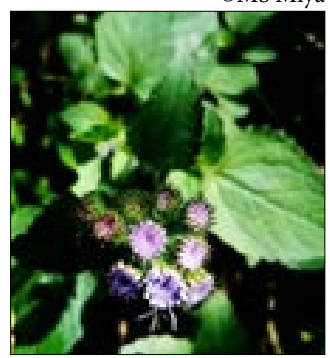

9. Ageratum houstonianum

(C) MS Miya

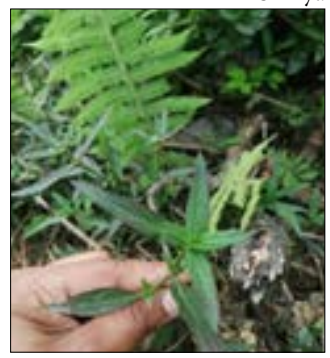

13. Alternatharia sessils

(C)MS Miya 


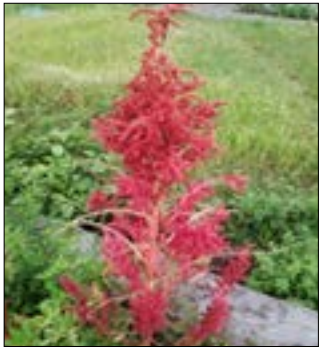

14. Amaranthus caudatus

CD Gautam

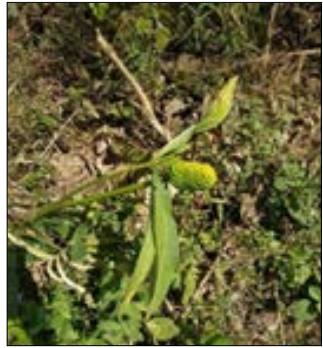

18. Arisaema speciosum

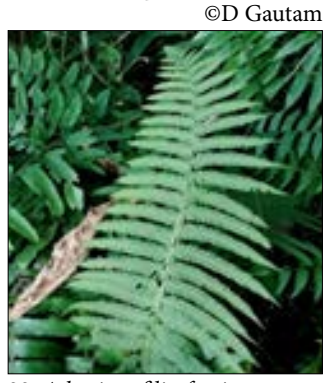

22. Athyrium filix-femina

(C)MS Miya

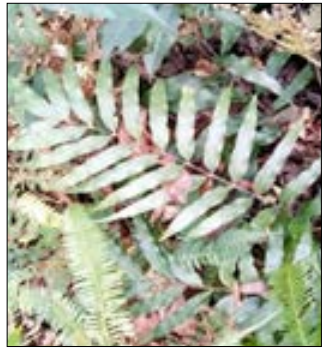

26. Blechnum corbassonii

(C)MS Miya

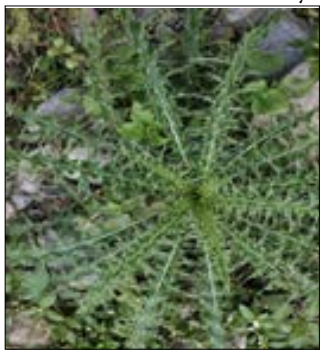

30. Carduus nutans

CD Gautam

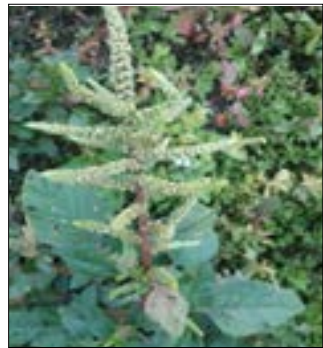

15. Amaranthus spinosus

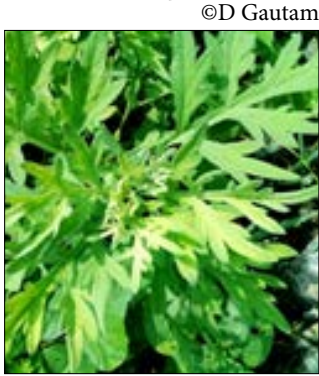

19. Artemisia indica

(C)MS Miya

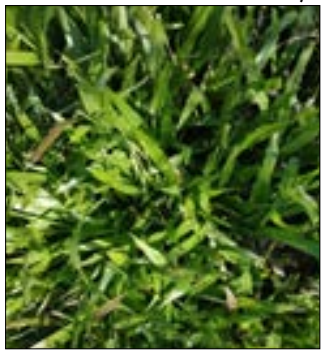

23. Axonopus compressus

(C) Gautam

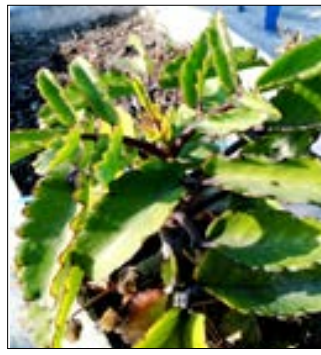

27. Bryophyllum pinnatum

(C)MS Miya

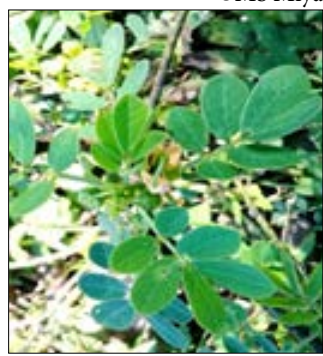

31. Cassia tora

(C)MS Miya

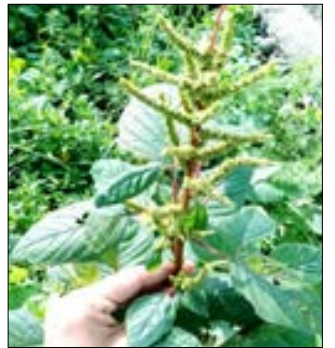

16. Amaranthus viridis

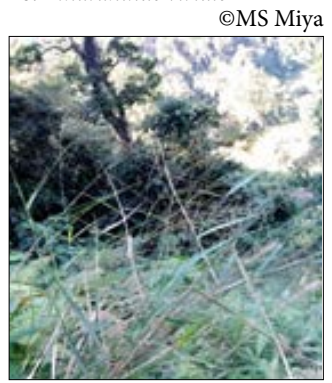

20. Arundinella nepalensi

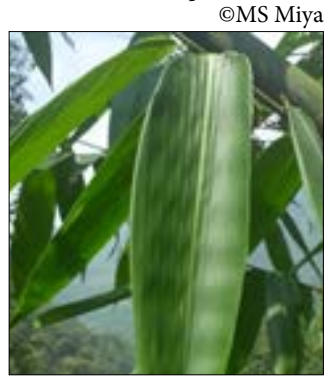

24. Bambusa sp.

(C)MS Miya

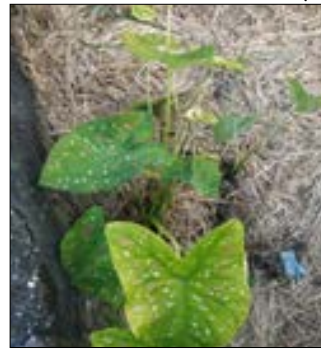

28. Caladium bicolor

(C)MS Miya

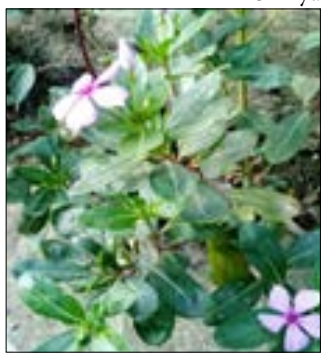

32. Catharanthus roseus

(C)MS Miya

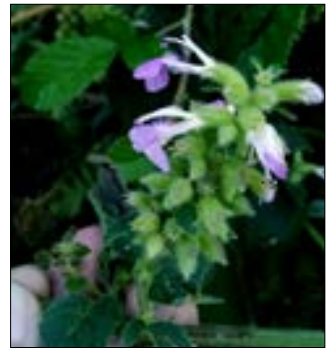

17. Anisomeles indica

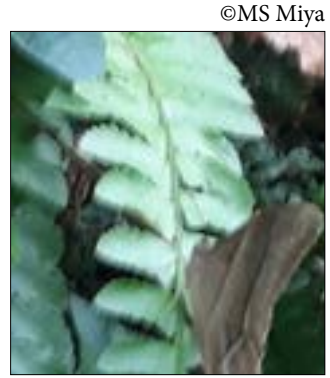

21. Asplenium normale

(C)MS Miya

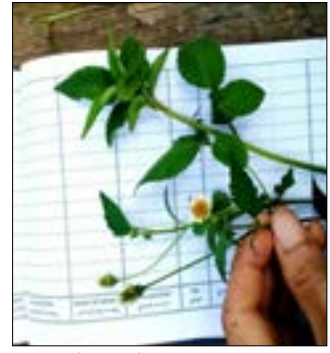

25. Bidens pilosa

(C)MS Miya

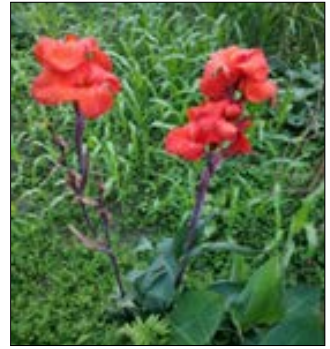

29. Canna indica

(c)D Gautam

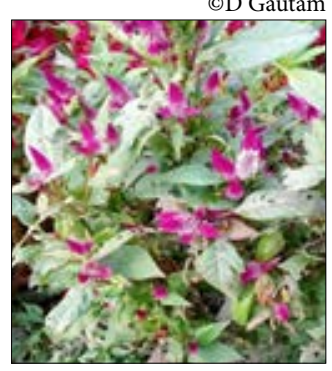

33. Celosia cristata

(C)MS Miya 


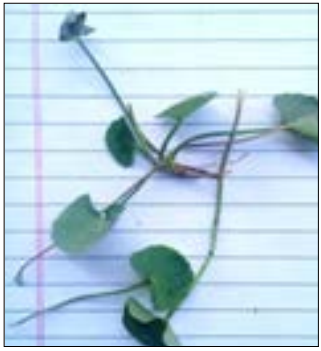

34. Centella asiatica $\odot$ MS Miya

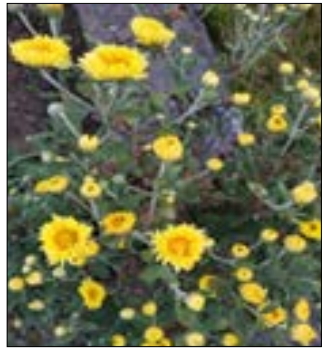

38. Chrysanthemum indicum

(C) Gautam

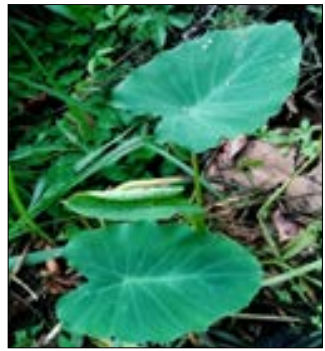

42. Colocasia esculenta

(C)MS Miya

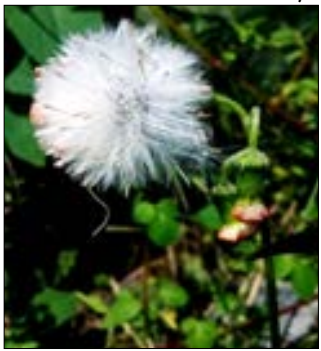

46. Crassocephalum crepidioides

(c)MS Miya

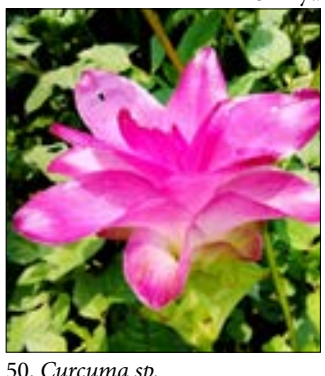

(C)MS Miya

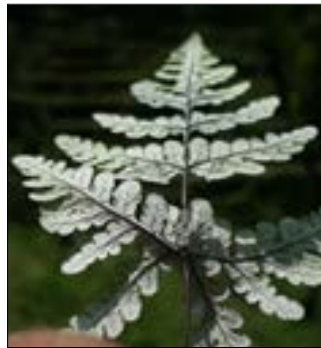

35. Cheilanthes bicolor

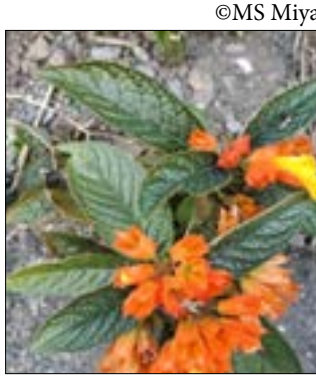

39. Chrysothemis pulchella

(c)D Gautam

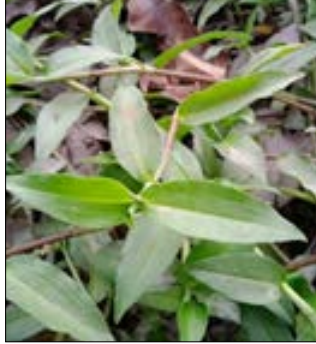

43. Commelina benghalensis

(C) D Gautam

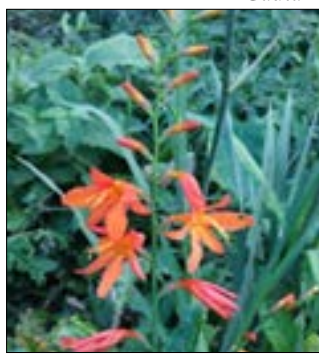

47. Crocosmia crocosmiiflora

(c)D Gautam

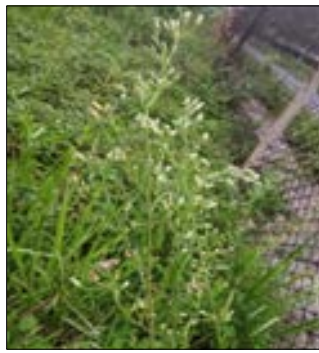

51. Cyanthillium cinereum

(CD Gautam

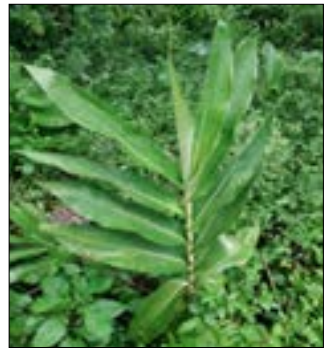

36. Cheilocostus speciosus

(c) G Gautam

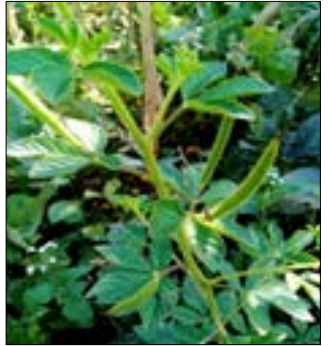

40. Cleome viscosa

(c)MS Miya

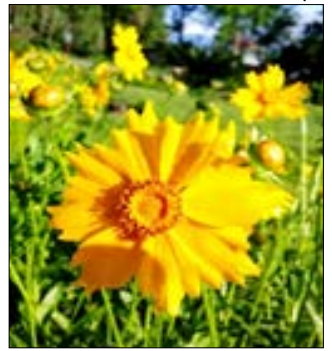

44. Coreopsis grandiflor

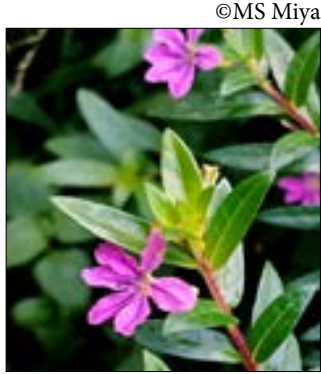

48. Cuphea hyssopifolia

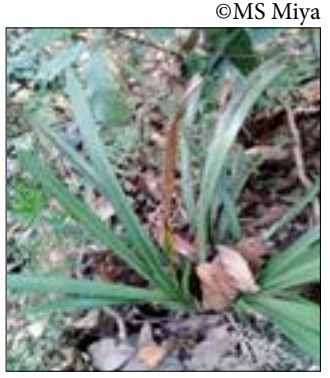

52. Cymbidium sp.

(c)MS Miya

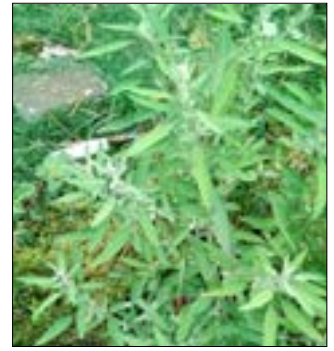

37. Chenopodium album

(c) Gautam

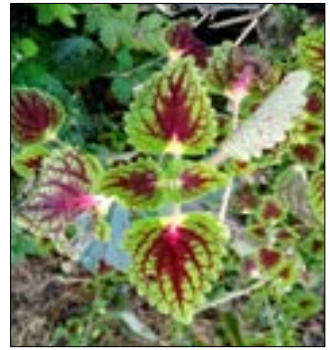

41. Coleus sp.

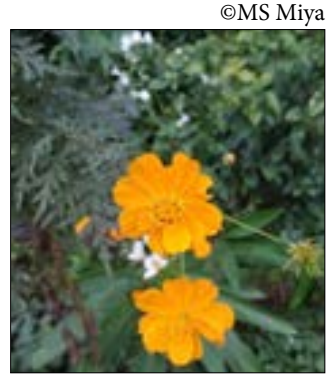

45. Cosmos sulphureus

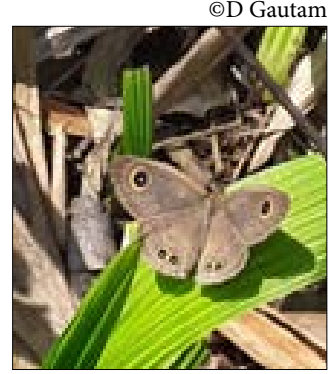

49. Curculigo sp.

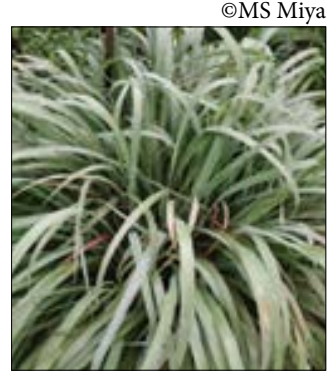

53. Cymbopogon citratus

(c)MS Miya 


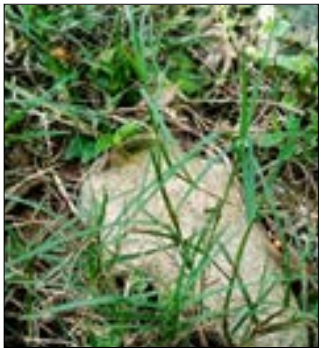

54. Cynodon dactylon

(C)MS Miya

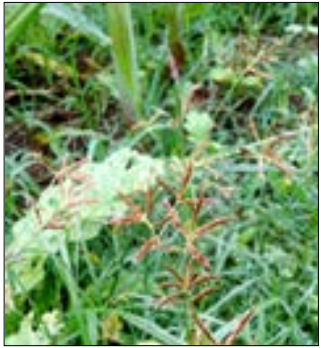

58. Cyperus rotundus

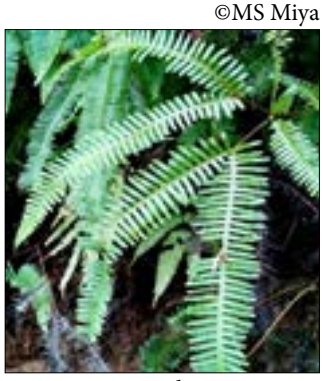

62. Dicranopteris linearis

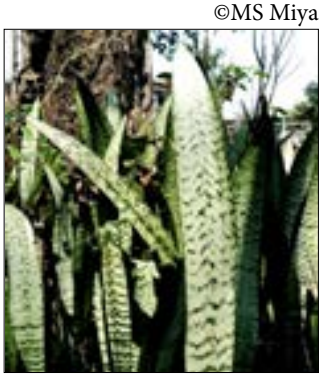

66. Dracaena trifasciata

(C)MS Miya

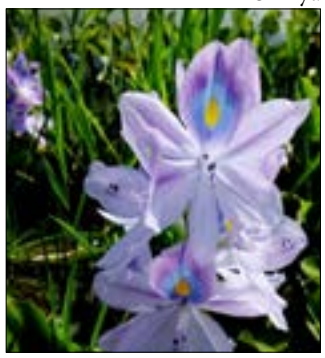

70. Eichhornia sp.

(C)MS Miya

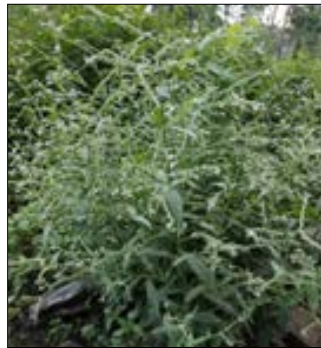

55. Cynoglossum lanceolatum

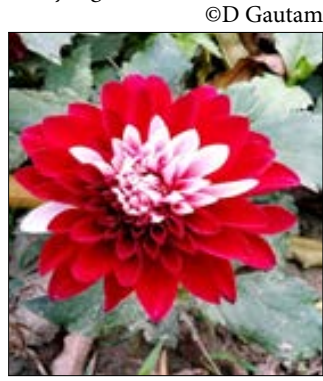

59. Dahlia $s p$

(C)MS Miya

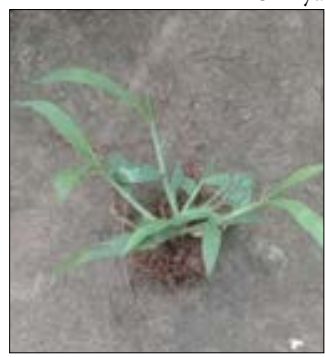

63. Digitaria sp.

(C)MS Miya

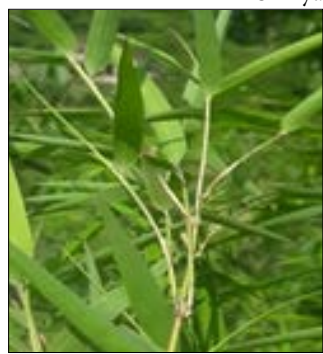

67. Drepanostachyum intermedium 68. Drymaria cordat

(C)MS Miya

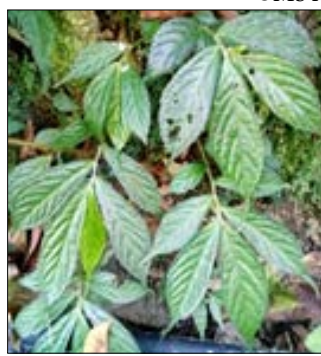

71. Elatostema $s p$

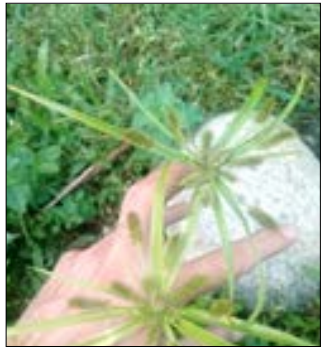

56. Cyperus cyperoides

(C)MS Miya

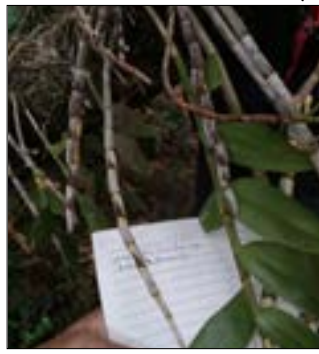

60. Dendrobium sp.

(C)MS Miya

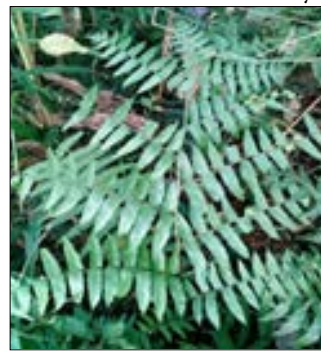

64. Diplazium esculentum

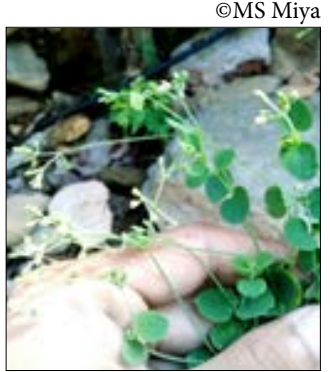

(C)MS Miya

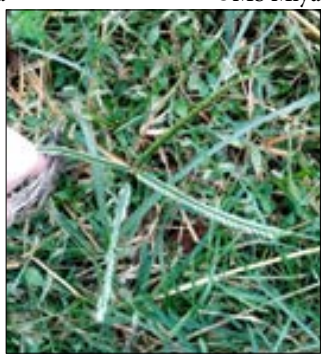

72. Eleusine indica

(C) MS Miya

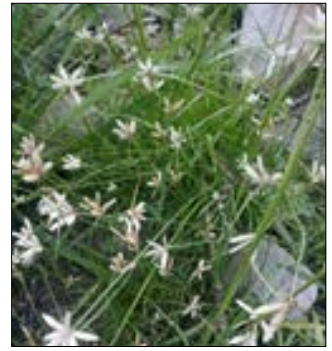

57. Cyperus niveus

CD Gautam

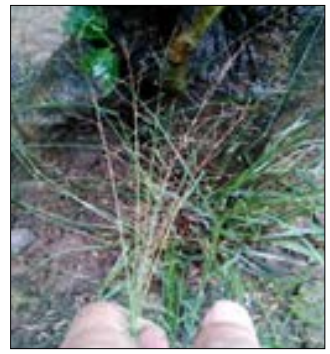

61. Desmostachya bipinnata

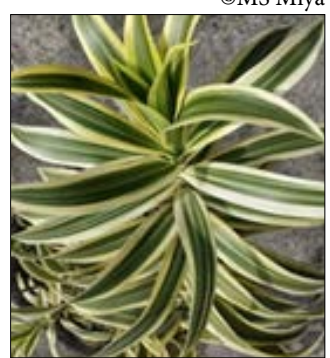

65. Dracaena reflexa

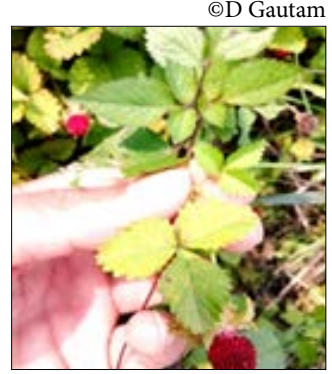

69. Duchesnea indica

(C) MS Miya

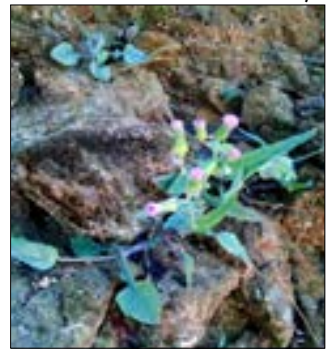

73. Emilia sonchifolia

(C)MS Miya 


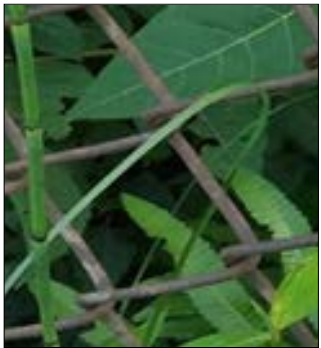

74. Equisetum debile

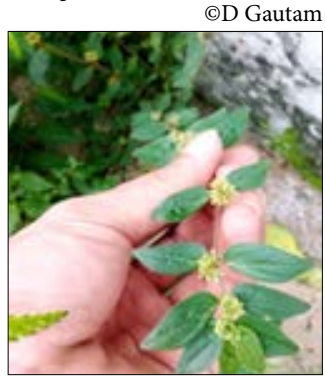

78. Euphorbia hirta (MS Miya

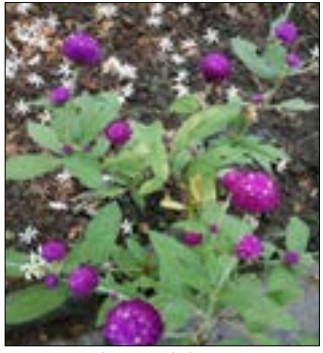

82. Gomphrena globosa

(OD Gautam

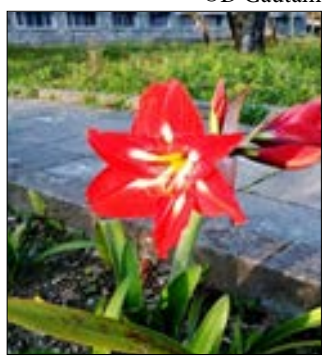

86. Hippeastrum sp.

(c)MS Miya

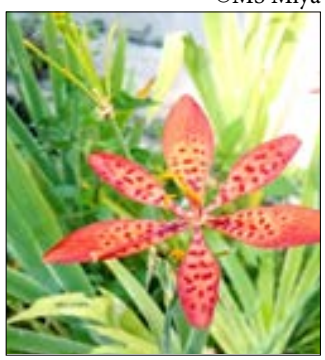

90. Iris domestica

(c)MS Miya

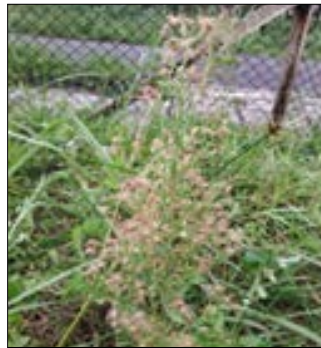

75. Erigeron canadensis

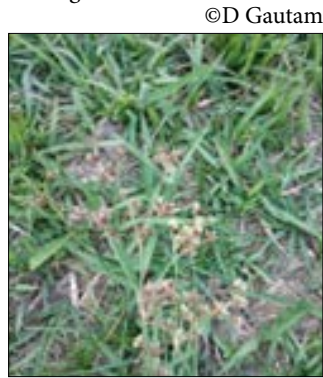

79. Fimbristylis falcato

(C) Gautam

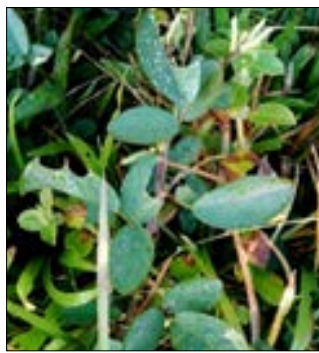

83. Grona heterocarpa

(C)MS Miya

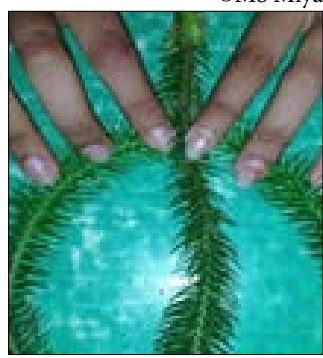

87. Huperzia squarrosa

(c)MS Miya

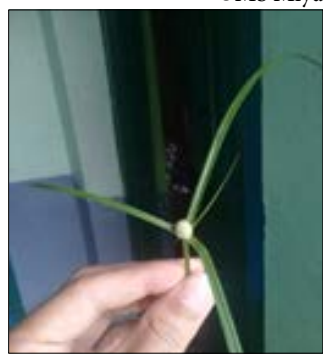

91. Kyllinga sp.

(c)MS Miya

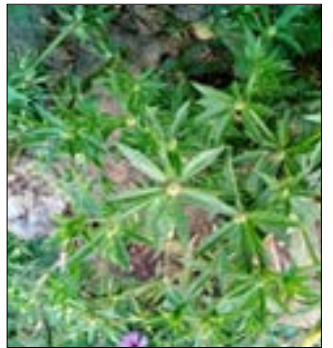

76. Eryngium foetidumm

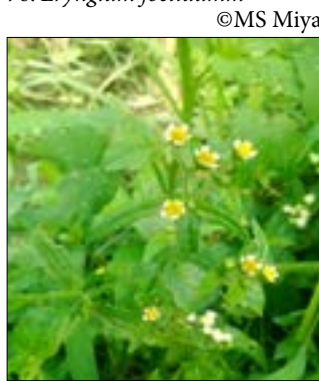

80. Galinsoga quadriradiato

()MS Miya

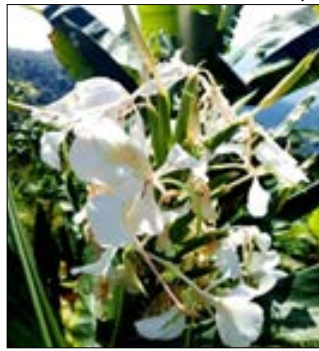

84. Hedychium coronarium

( ) MS Miya

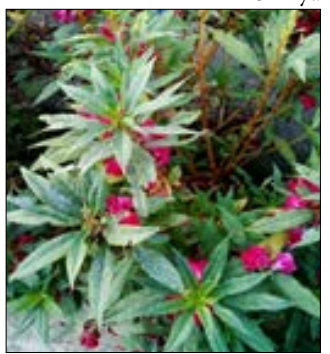

88. Impatiens balsamina

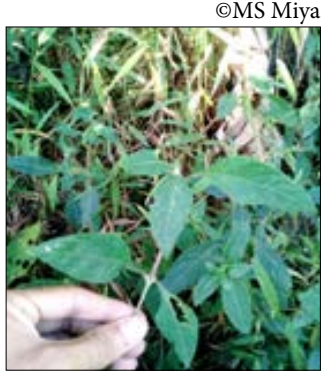

92. Lepidagathis incurva

○MS Miya

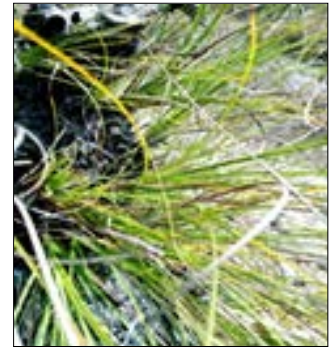

77. Eulaliopsis binat

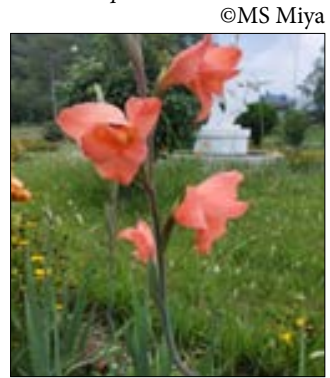

81. Gladiolus daleni

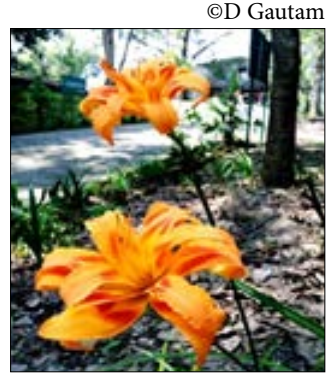

85. Hemerocallis fulva

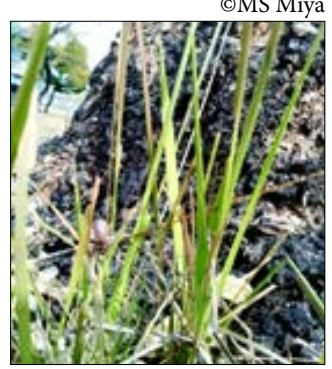

89. Imperata cylindrica

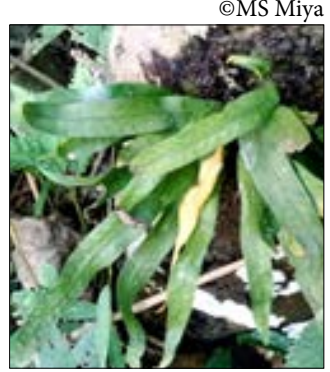

93. Lepisorus nudus

()MS Miya 


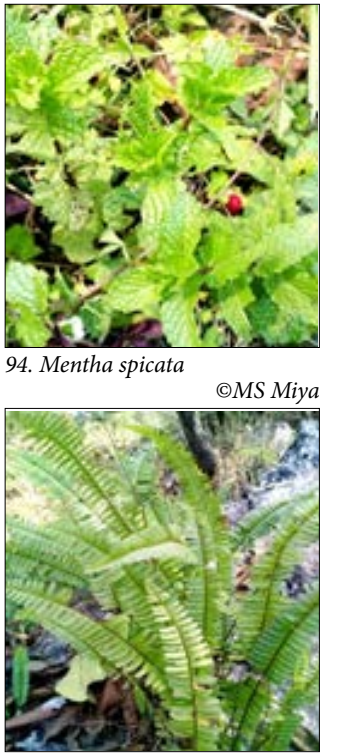

98. Nephrolepis cordifolia

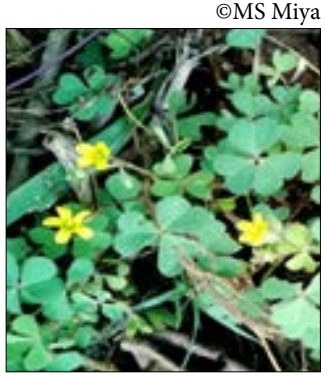

102. Oxalis corniculata

(C) MS Miya

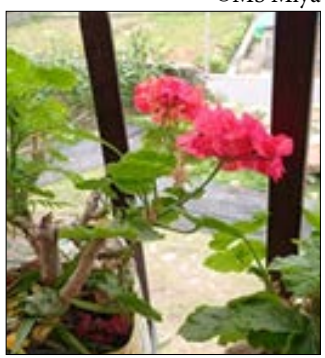

106. Pelargonium $s p$

() MS Miya

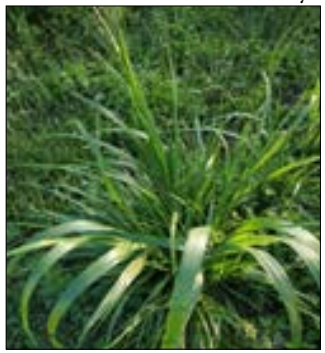

110. Phragmites karka

(c) G Gautam

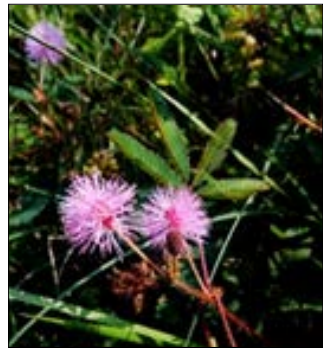

95. Mimosa pudica

(c)MS Miya

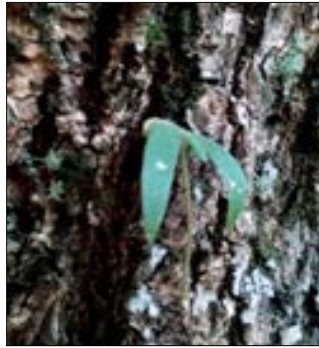

99. Oberonia pachyrachis

@MS Miya

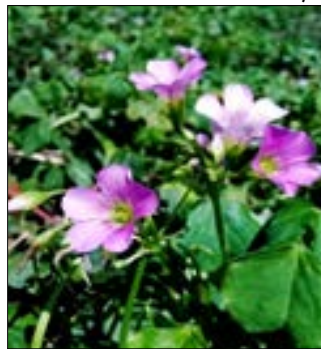

103. Oxalis latifolia

(c)MS Miya

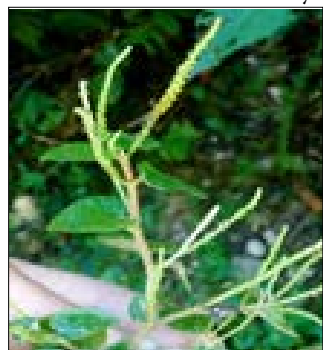

107. Peperomia pellucida

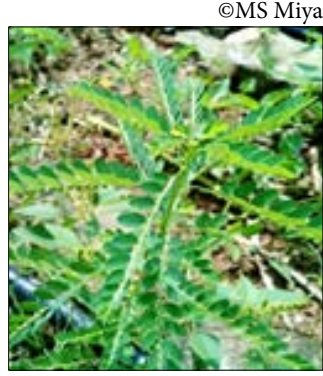

111. Phyllanthus nirur

(c)MS Miya

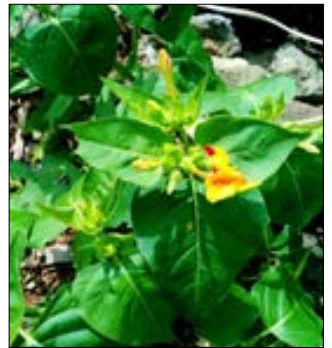

96. Mirabilis jalapa

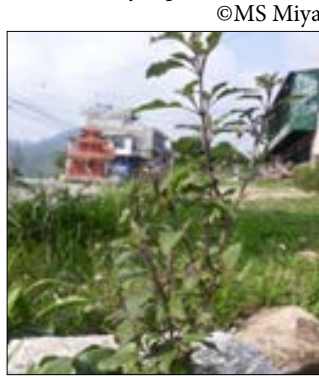

100. Ocimum tenuiflorum

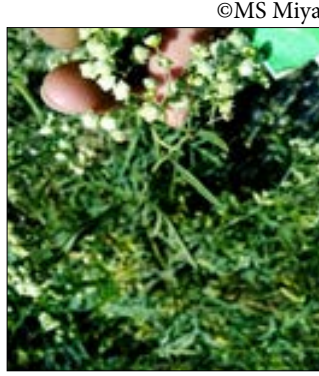

104. Parthenium hysterophorus

(c)MS Miya

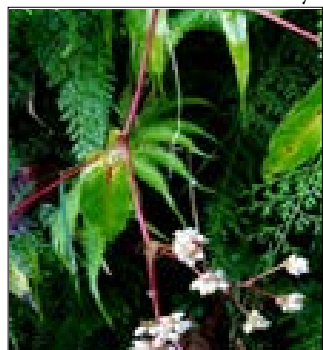

108. Persicaria chinensis

(c)MS Miya

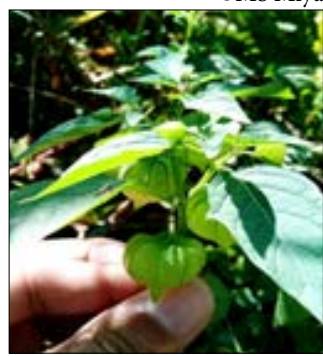

112. Physalis sp.

(c) Miya

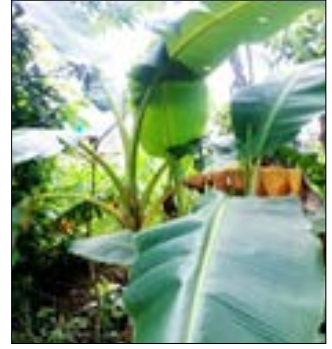

97. Musa paradisiaca

() MS Miya

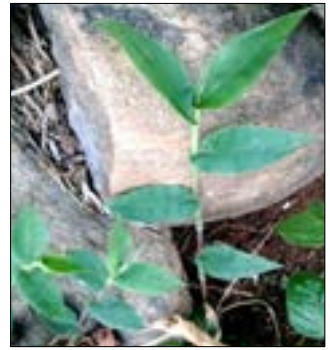

101. Oplismenus hirtellu

(c)MS Miya

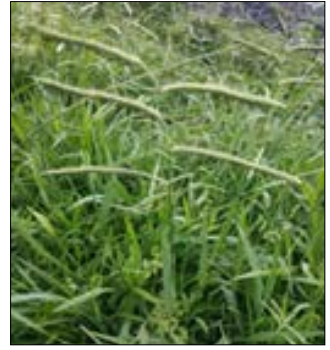

105. Paspalum dilatatum

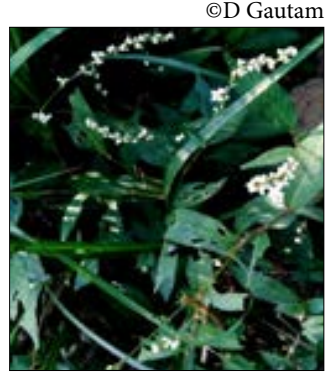

109. Persicaria pubescen

(c)MS Miya

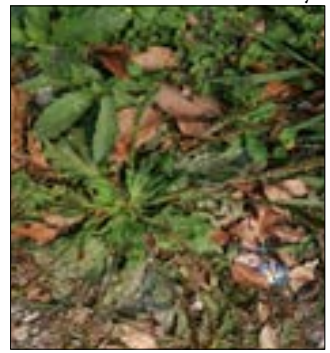

113. Plantago $s p$

()MS Miya 


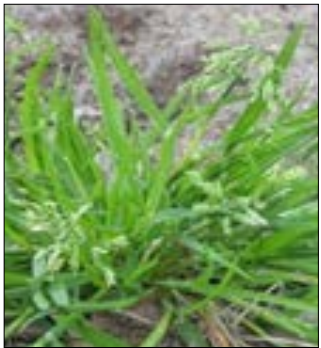

114. Poa annua $\odot \mathrm{D}$ Gautam

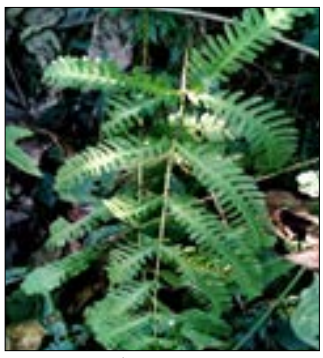

118. Pteris dispar @MS Miya

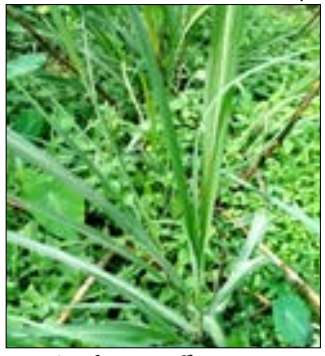

122. Saccharum officinarum

(c)MS Miya

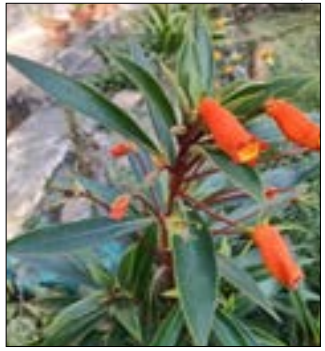

126. Seemannia sylvatica

(c) Gautam

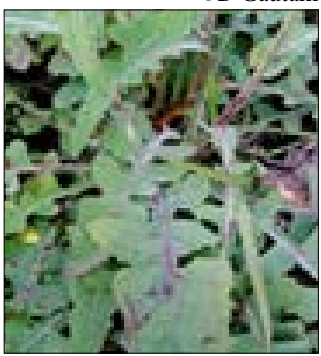

130. Sonchus oleraceus

(c)MS Miya

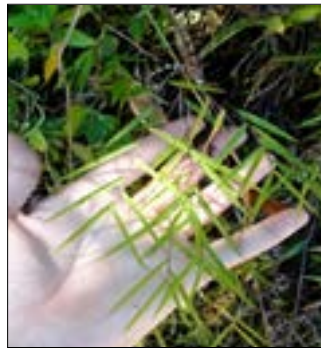

115. Pogonatherum paniceum

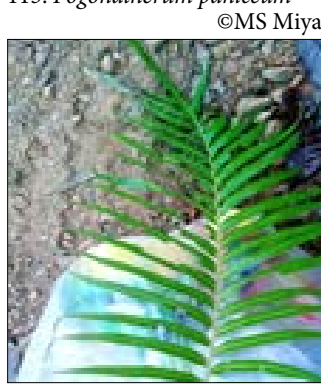

119. Pteris vittata

()MS Miya

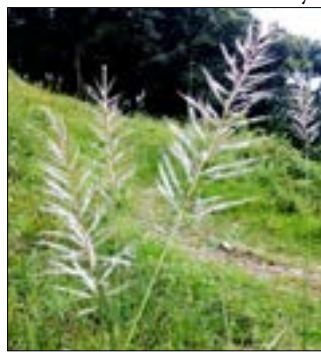

123. Saccharum spontaneum

(c)MS Miya

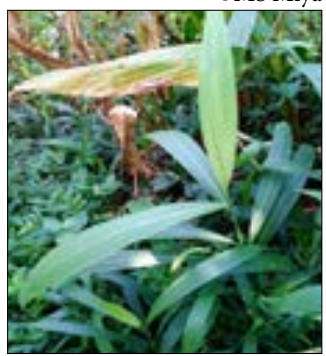

127. Setaria palmifolia

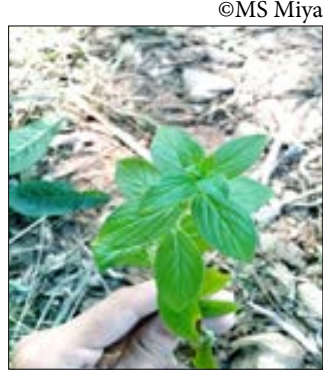

131. Spermacoce latifolia

(c)MS Miya

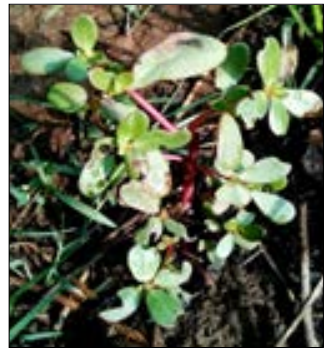

116. Portulaca oleracea

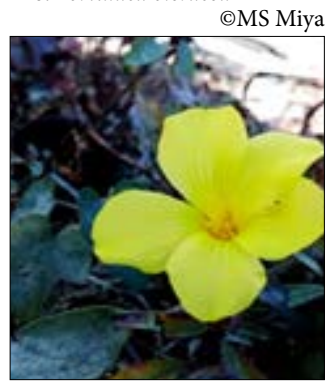

120. Reinwardtia indica

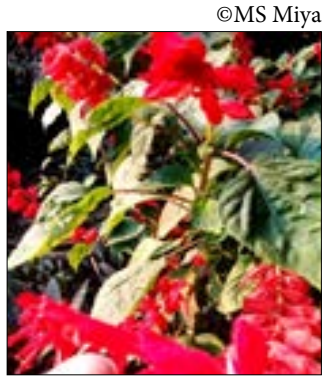

124. Salvia splendens

(C)MS Miya

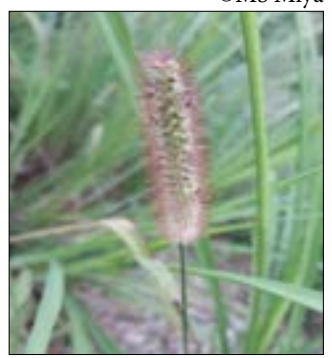

128. Setaria pumila

( )MS Miya

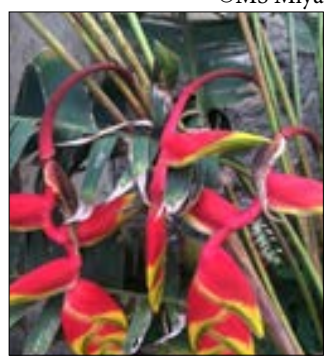

132. Strelitzia reginae

○MS Miya

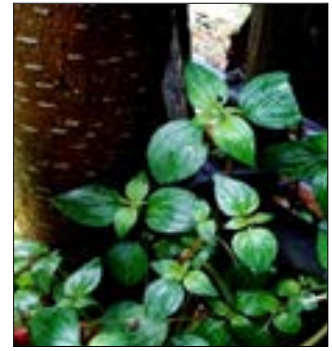

117. Pouzolzia zeylanica

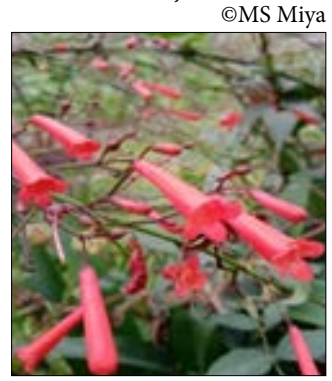

121. Russelia equisetiformis

oD Gautam

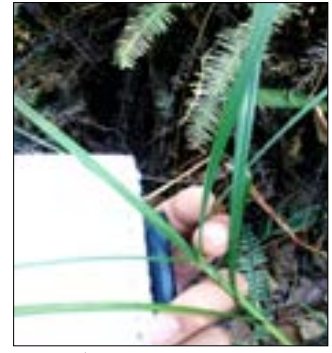

125. Scleria terrestris

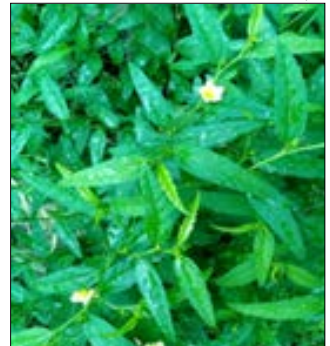

129. Sida cordifolia

()MS Miya

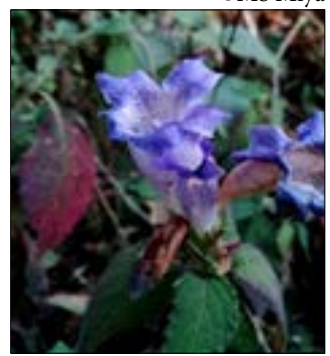

133. Strobilanthes $s p$.

()MS Miya 


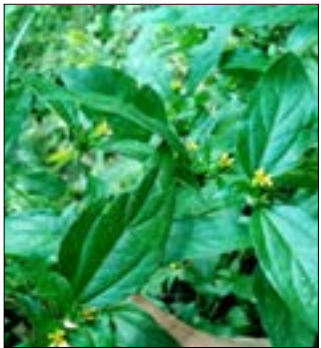

134. Synedrella nodiflora

(c)MS Miya

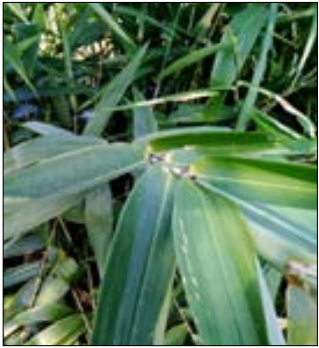

138. Thysanolaena maxima

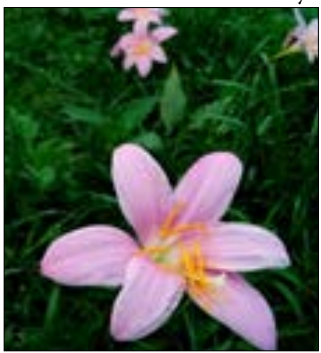

142. Zephyranthes carinato

(C) MS Miya

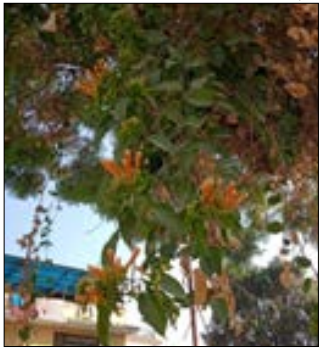

3. Campsis radicans

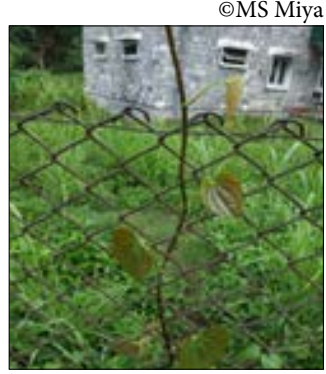

7. Dioscorea bulbifera

(c) D Gautam
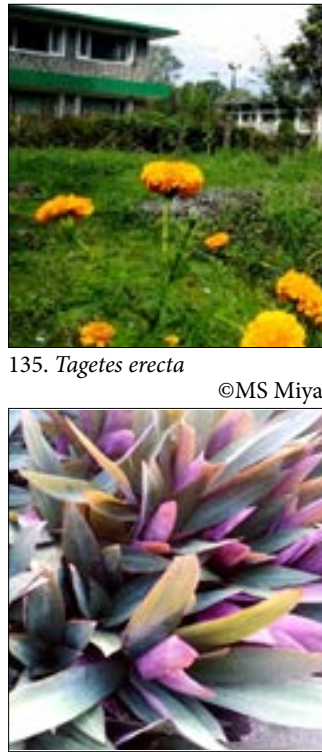

139. Tradescantia pallida

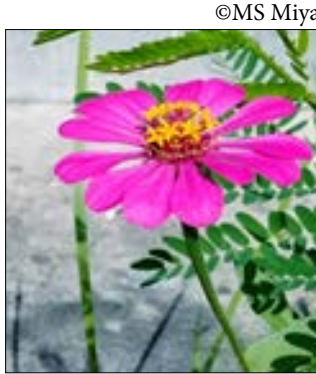

143. Zinnia elegans

(C)MS Miya

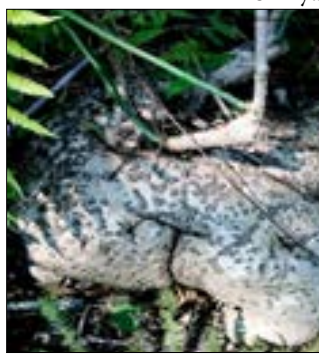

4. Cissampelos pareira

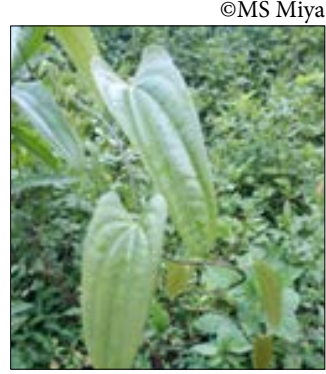

8. Dioscorea deltoidea

(C) Gautam

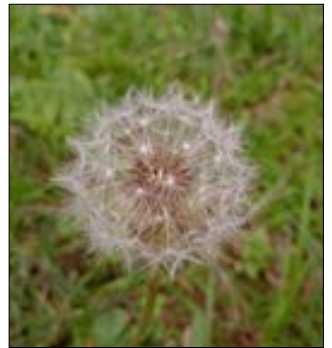

136. Taraxacum $s p$.

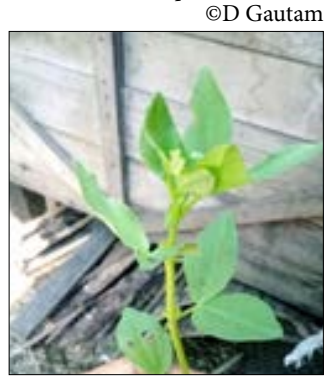

140. Vicia faba

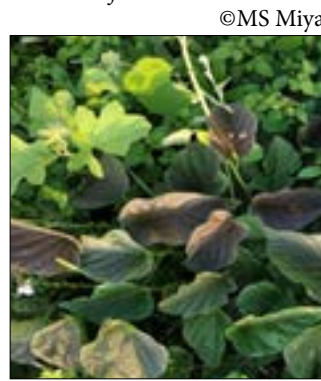

1. Argyreia nervosa

() MS Miya

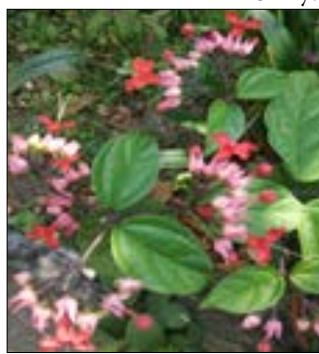

5. Clerodendrum thomsoniae

(c)D Gautam

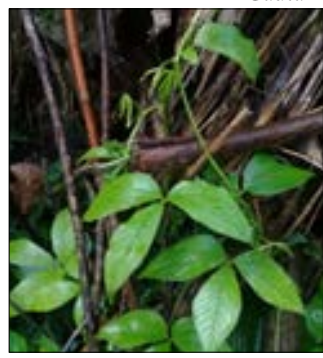

9. Dioscorea kamoonensis

()MS Miya

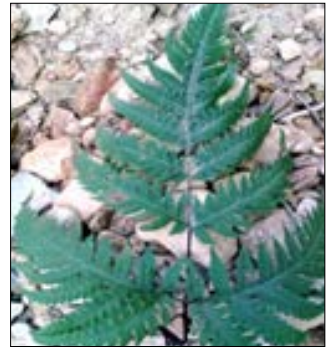

137. Tectaria macrodonta

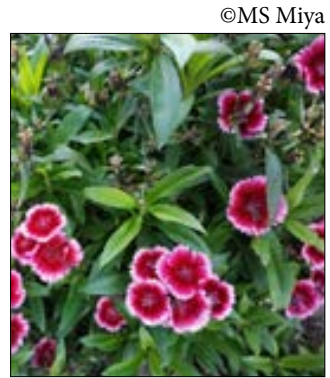

141. Viola $s p$

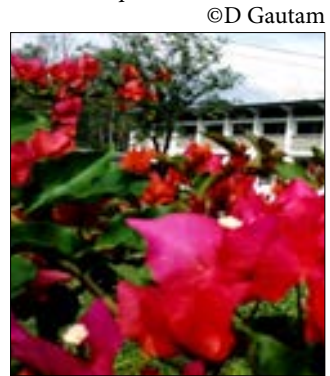

2. Bougainvillea spectabilis

(C)MS Miya

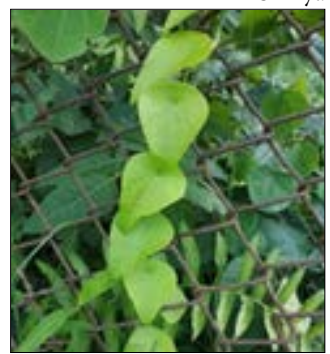

6. Cyclea sp.

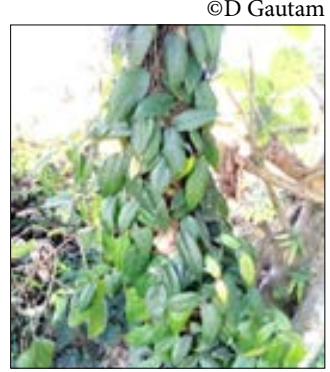

10. Ficus pumila

(c)MS Miya 


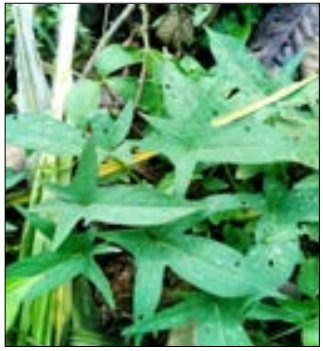

11. Ipomoea batatas

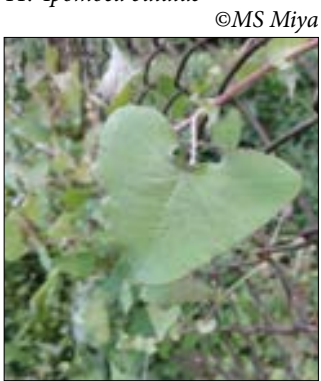

15. Persicaria perfoliata

(c) D Gautam

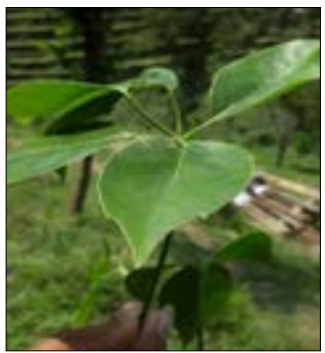

19. Schefflera venulosa

(C)MS Miya

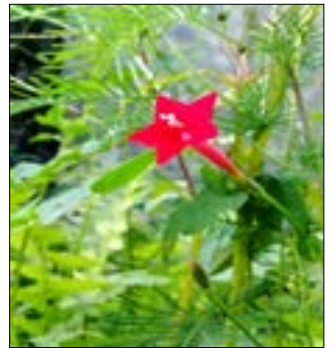

12. Ipomoea quamoclit

(C) MS Miya

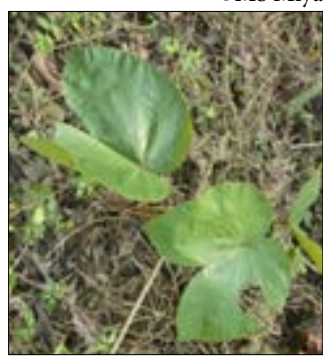

16. Phanera vahlii

(C)MS Miya

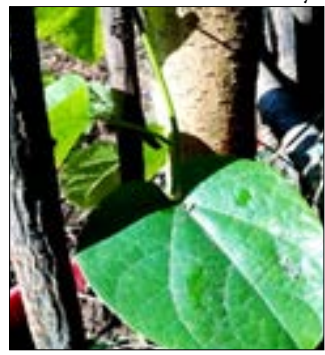

20. Tinospora cordifolia

(c) MS Miya

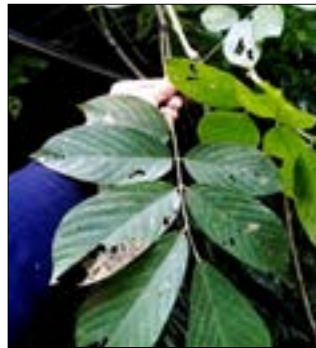

13. Millettia extensa

(C)MS Miya

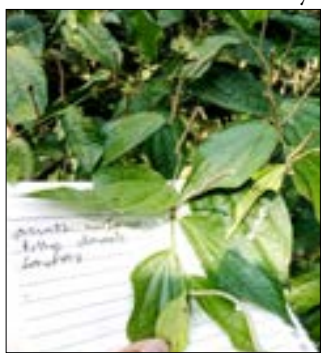

17. Piper longum

(C)MS Miya

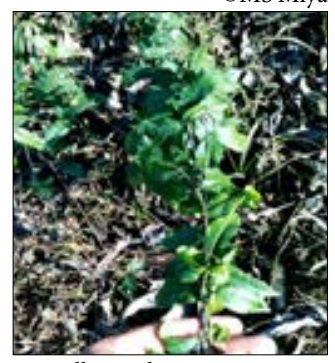

21. Vallaris solanacea

(C)MS Miya

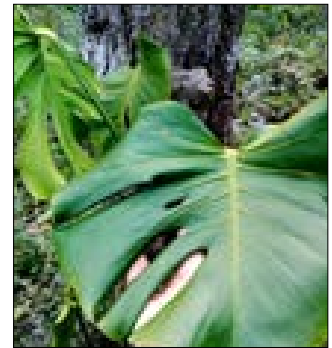

14. Monstera deliciosa (C)MS Miya

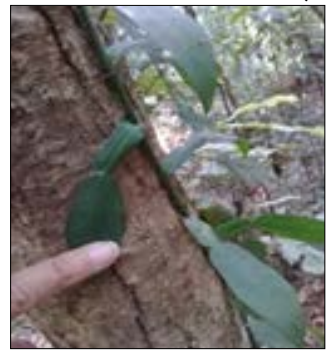

18. Pothos chinensis

(C)MS Miya 